\title{
A Self-regularization Technique in Boundary Element Method for Three-dimensional Elastostatics
}

\author{
by \\ Menggu He \\ A Thesis submitted to \\ the Faculty of Graduate Studies and Research \\ in partial fulfilment of \\ the requirements for the degree of \\ Master of Applied Science \\ in \\ Department of Mechanical and Aerospace Engineering \\ Carleton University \\ Ottawa, Ontario, Canada
}

April 2013

Copyright (C)

2013 - Menggu He 
Library and Archives

Canada

Published Heritage

Branch

395 Wellington Street

Ottawa ON K1A ON4

Canada
Bibliothèque et

Archives Canada

Direction du

Patrimoine de l'édition

395 , rue Wellington

Ottawa ON K1A ON4

Canada
Your file Votre référence

ISBN: 978-0-494-94643-5

Our file Notre référence

ISBN: $978-0-494-94643-5$
NOTICE:

The author has granted a nonexclusive license allowing Library and Archives Canada to reproduce, publish, archive, preserve, conserve, communicate to the public by telecommunication or on the Internet, loan, distrbute and sell theses worldwide, for commercial or noncommercial purposes, in microform, paper, electronic and/or any other formats.

The author retains copyright ownership and moral rights in this thesis. Neither the thesis nor substantial extracts from it may be printed or otherwise reproduced without the author's permission.
AVIS:

L'auteur a accordé une licence non exclusive permettant à la Bibliothèque et Archives Canada de reproduire, publier, archiver, sauvegarder, conserver, transmettre au public par télécommunication ou par l'Internet, prêter, distribuer et vendre des thèses partout dans le monde, à des fins commerciales ou autres, sur support microforme, papier, électronique et/ou autres formats.

L'auteur conserve la propriété du droit d'auteur et des droits moraux qui protege cette thèse. $\mathrm{Ni}$ la thèse ni des extraits substantiels de celle-ci ne doivent être imprimés ou autrement reproduits sans son autorisation.
In compliance with the Canadian Privacy Act some supporting forms may have been removed from this thesis.

While these forms may be included in the document page count, their removal does not represent any loss of content from the thesis.
Conformément à la loi canadienne sur la protection de la vie privée, quelques formulaires secondaires ont été enlevés de cette thèse.

Bien que ces formulaires aient inclus dans la pagination, il n'y aura aucun contenu manquant. 


\section{Abstract}

The Boundary Element Method (BEM), based on the displacement Boundary Integral Equation (BIE), is now well established as an efficient numerical tool for engineering analysis. However, in some linear elastostatic problems, if the distance from an interior point to the surface of the solution domain, or between two points on two parts of the surface close by in a thin, slender body, is relatively small, it will give rise to near singularities of the kernel functions of the BIE as they are singular with respect to this distance. For these problems, the conventional BEM is not well suited as very siginificant mesh refinement becomes necessary. In this study, a self-regularization BEM scheme previously applied in two-dimensional (2D) elastostatics is extended to three dimensions (3D). It is first implemented for the evaluation of the displacements and stresses at an interior point. Several numerical examples are presented to demonstrate the veracity of the scheme, for accurate solutions are obtained even for interior points very close to the surface without using refined mesh. A similar selfregularized traction-BIE extended from the corresponding $2 \mathrm{D}$ scheme to the $3 \mathrm{D}$ case is then investigated for the thin-body problems. It is not met with success, however, due to the lack of $C^{1, \alpha}$ continuity across element boundaries. A new self-regularized displacement-BIE which does not require this continuity requirement is developed for treating the thin-body problem. Using this scheme, preliminary tests show that very accurate results can still be obtained for the boundary stresses and displacements even when the cross section of the domain is very thin indeed. More extensive tests will still be necessary to fully establish the general applicability of this new scheme. 


\section{Acknowledgments}

I would like to acknowledge my supervisor, Dr. Choon-Lai Tan, for his endless help both academically and mentally. He has led me into the wonderful world of research and constantly provides me with invaluable directions and decisions. My thesis would never be completed without his unreserved support. I am grateful to the faculty and staff of the Department of Aerospace and Mechanical Engineering at Carleton University for their academic support and resources. Great thanks to my parents, who have given me continuous support and encouragement during my graduate study, and have kept me optimistic about research and life the whole time. 


\section{Table of Contents}

$\begin{array}{ll}\text { Abstract } & \text { iii }\end{array}$

Acknowledgments $\quad$ iv

Table of Contents $\quad$ v

List of Tables $\quad$ viii

$\begin{array}{ll}\text { List of Figures } & \text { ix }\end{array}$

List of Symbols $\quad$ xii

1 Introduction $\quad 1$

2 Review of Boundary Element Method $\quad 5$

2.1 Introduction . . . . . . . . . . . . . . . . 5

2.2 The Boundary Element Method in

Elastostatics . . . . . . . . . . . . . . . . 6

2.3 Boundary Integral Equations . . . . . . . . . . . . . 8

2.4 Numerical Integration . . . . . . . . . . . . . . . . . . . . . 9

2.5 Stress and Displacement Solution for Interior Points . . . . . . . . . . 16

2.6 Traction-BIE and Hypersingular-BIE . . . . . . . . . . . . . . 19

2.7 Concluding Remarks . . . . . . . . . . . . . . . . . . . . 23 
3 Self-regularization for Interior Points $\quad 27$

3.1 Introduction . . . . . . . . . . . . . . . . 27

3.2 Self-regularization of Interior Points in Three Dimensions . . . . . . . 27

3.3 Numerical Examples ... . . . . . . . . . . . . . . 33

3.4 Discussion of Results . . . . . . . . . . . . . . . . . . . . . . . . . 38

3.5 Summary ........................ . . 40

4 Self-regularized Traction-BIE $\quad 65$

4.1 Introduction . . . . . . . . . . . . . . . . . 65

4.2 Self-regularized Traction-BIE . . . . . . . . . . . . . . . . 65

4.3 Treatments of Displacement-gradient Discontinuity . . . . . . . . . 70

4.3.1 Relaxed Continuity . . . . . . . . . . . . . . . 70

4.3 .2 Collocation Strategy . . . . . . . . . . . . . . . . 71

4.4 Numerical Examples . . . . . . . . . . . . . . . . 72

4.4.1 Thin Plate under Uniform Stress . . . . . . . . . . . . 72

4.4.2 Thick-walled Cylinder under Axial Load and Internal Pressure 73

4.4.3 Internally Pressurized Sphere . . . . . . . . . . . 74

4.4 .4 Discussion . . . . . . . . . . . . . . . . 74

4.5 Summary . . . . . . . . . . . . . . . . . 76

5 Self-regularized Displacement-BIE for Thin-body Problems $\quad 89$

5.1 Introduction . . . . . . . . . . . . . . . . . . . 89

5.2 Self-regularized Displacement-BIE . . . . . . . . . . . . . . 89

5.3 Evaluation of the Free Terms $C_{i j} \ldots \ldots \ldots$. . . . . . . . . . . 92

5.4 Numerical Examples ... . . . . . . . . . . . . . 94

5.4.1 Thin Plate under Uniform Stresses . . . . . . . . . . . . . 94

5.4.2 Thin-walled Cylinder and Sphere . . . . . . . . . . 95

5.5 Concluding Remarks . . . . . . . . . . . . . . . . . . . 97 
6 Conclusion $\quad 113$

6.1 Suggestions for Future Work . . . . . . . . . . . . . 115

$\begin{array}{lr}\text { References } & 116\end{array}$ 


\section{List of Tables}

3.1 Interior point stress results for the cube under tensile stresses - Problem

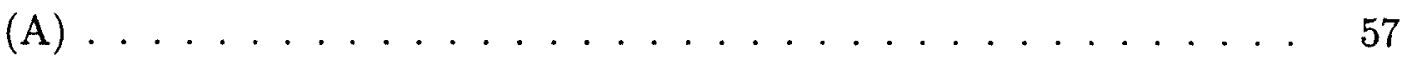

3.2 Interior point stress results for the cube under shear stresses - Problem

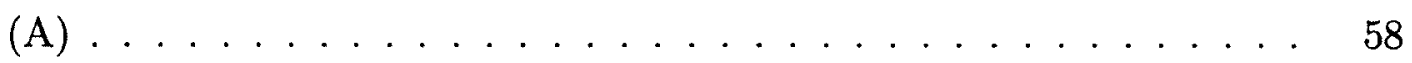

3.3 Interior point displacement results for the cube under tensile stresses - Problem (A) . . . . . . . . . . . . . . . . . . . . . .

3.4 Interior point displacement results for the cube under shear stresses Problem $(\mathrm{A}) \ldots \ldots \ldots \ldots \ldots \ldots \ldots$

3.5 Results for the cantilever beam - Problem (B) . . . . . . . . . . 61

3.6 Results of the internally pressurized cylinder - Problem (C) . . . . . 62

3.7 Results of the internally pressurized sphere - Problem (C) . . . . . 63

3.8 Stress concentration factors obtained from BEM and FEM solutions Problem $(\mathrm{D}-2) \ldots \ldots \ldots \ldots \ldots \ldots$

4.1 Stress results for the thick-walled cylinder under axial load . . . . 86

4.2 Stress results for the 1/4-model of the thick-walled cylinder under internal pressure $\ldots \ldots \ldots \ldots \ldots \ldots \ldots \ldots$

4.3 Stress results for the fully-modeled thick-walled cylinder under internal

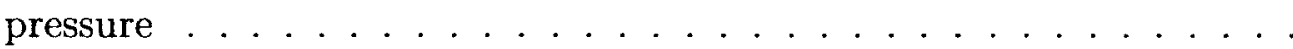




\section{List of Figures}

2.1 Typical BEM and FEM meshes for 2D and 3D problems . . . . . 24

2.2 Boundary conditions imposed on surface of domain $\ldots \ldots \ldots 25$

2.3 Local coordinate system of quadrilateral and triangular elements . . 26

3.1 Schematic of regularization point $\ldots \ldots \ldots 41$

3.2 Locating of the regularization point . . . . . . . . . . . 42

3.3 Schematic of the cubic body - Problem (A) . . . . . . . . . . 43

3.4 Definition and mesh of the cantilever beam - Problem (B) . . . . . 44

3.5 Interior point locations and meshes of the pressurized vessels - Problem

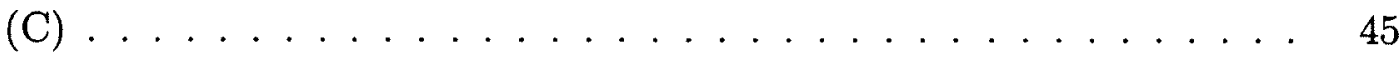

3.6 Schematic diagram of the double-notched bar - Problem (D-1) . . . 46

3.7 The modeled region of the double-notched bar - Problem (D-1) . . . 47

3.8 Boundary element mesh of the double-notched bar - Problem (D-1) . 48

3.9 Finite element mesh of the double-notched bar - Problem (D-1) . . 49

3.10 Stress variation along thickness of the double-notched bar - Problem

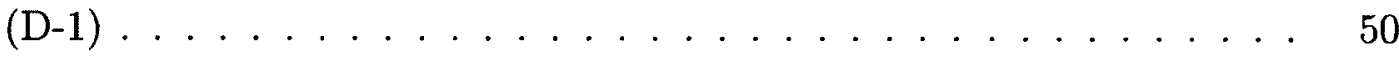

3.11 Stress variation at interior of the double-notched bar - Problem (D-1) 51

3.12 A cylinder with a spherical cavity - Problem (D-2) . . . . . . . . 52

3.13 BEM mesh of the cylindrical bar with a spherical cavity - Problem (D-2) 53

3.14 FEM mesh of the cylindrical bar with a spherical cavity - Problem (D-2) 54

3.15 Analytical solution for the cylinder with spherical cavity [Pilkey, 2008] 55 
3.16 Stress distribution at the interior points of the cylinder with cavity by BEM and FEM - Problem (D-2) . . . . . . . . . . . . 56

4.1 Location of the collocation points inside an element . . . . . . . . 77

4.2 Variation of the maximum error in stresses with thickness for the thin

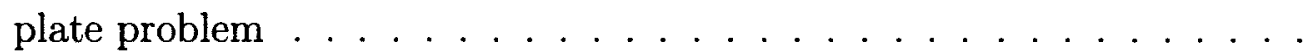

4.3 Variation of the maximum error in displacements with thickness for the thin plate problem . . . . . . . . . . . . . . 79

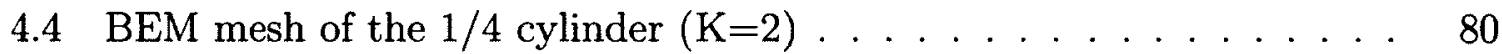

4.5 BEM mesh of the fully-modeled cylinder $(\mathrm{K}=2) \ldots \ldots 1$

4.6 BEM mesh of the fully-modeled sphere $(\mathrm{K}=2) \ldots \ldots 2$

4.7 Variation of maximum error in stresses with radius ratio of the hollow

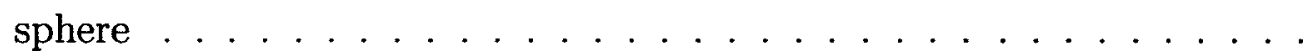

4.8 Variation of maximum error in displacements with radius ratio of the hollow sphere . . . . . . . . . . . . . . . . . 84

4.9 Schematic of the interior point between two surfaces . . . . . . . . 85

5.1 Schematic of elements sharing source point $P$. . . . . . . . . 99

5.2 Illustrative figure for solid angle $\psi \ldots$. . . . . . . . . . . . . . 100

5.3 Boundary element mesh of the thin plate problem . . . . . . . . . 101

5.4 Variation of the percentage errors of the numerical results for the thinplate problem with thickness . . . . . . . . . . . . . 102

5.5 Initial boundary element mesh of the thin-walled cylinder . . . . . . . 103

5.6 Percentage errors in the stresses of the pressurized thin-walled cylinder problem $(t=0.1) \ldots \ldots \ldots \ldots$. . . . . . . . . . . . 104

5.7 Percentage errors in the stresses of the pressurized thin-walled cylinder problem $(\mathrm{t}=0.01) \ldots \ldots . \ldots \ldots 5$ 
5.8 Percentage errors in the stresses of the pressurized thin-walled cylinder problem $(\mathrm{t}=0.001) \ldots \ldots \ldots \ldots$

5.9 Refined mesh of the thin-walled cylinder . . . . . . . . . . . 107

5.10 Percentage errors in the stresses in the refined BEM mesh for the thin-

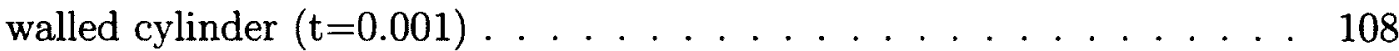

5.11 Variation of percentage errors in radial stress from BEM and FEM solutions with aspect ratio of elements across thickness . . . . . . 109

5.12 Partially refined mesh for the case of $t=0.1 \ldots \ldots 110$

5.13 BEM mesh of the thin-walled sphere problem . . . . . . . . . 111

5.14 Variation of the maximum errors in the circumference stress for the pressurized thin-walled sphere problem with the thickness ratio $K$. . 112 


\section{List of Symbols}

\section{Symbols Definition}

[A] system matrix storing coefficients for displacements

$[\bar{A}] \quad$ system matrix storing coefficients for unknowns

[B] system matrix storing coefficients for tractions

\{b\} system vector containing known values

BEM boundary element method

BIE boundary integral equation

$C_{i j} \quad$ free terms at diagonal of the system matrix, $\mathrm{i}, \mathrm{j}=1,3$

$D_{k i j} \quad$ kernel of Somigliana's identity for stresses, $\mathrm{i}, \mathrm{j}=1,3$

$E \quad$ Young's modulus

FEM finite element method

$J \quad$ Jacobian of transformation

$K \quad$ radius ratio

$N_{c} \quad$ shape functions

xii 


\begin{tabular}{ll}
$n_{i}$ & unit outward normal, $\mathrm{i}=1,3$ \\
$p$ & interior source point \\
$P$ & source point or load point on the boundary \\
$P^{d(b, c)}$ & c-th node of the $d$-th element \\
$P^{a}$ & a-th node of the boundary mesh \\
$Q$ & field point on the boundary \\
$r$ & distance between source point and field point; radius \\
$S$ & boundary of the problem domain \\
$S_{k i j}$ & kernel of Somigliana's identity for stresses, $\mathrm{i}, \mathrm{j}=1,3$ \\
$T_{i j}$ & fundamental solution for tractions, $\mathrm{i}, \mathrm{j}=1,3$ \\
$t_{i}$ & traction components, $\mathrm{i}=1,3$ \\
$\eta_{i}$ & intrinsic coordinates of the triangular sub-elements, $\mathrm{i}=1,2$ \\
$u_{i, j}$ & displacement gradients, $\mathrm{i}, \mathrm{j}=1,3$ \\
$U_{i j}$ & fundamental solution for displacements, $\mathrm{i}, \mathrm{j}=1,3$ \\
$u_{i}$ & displacement components, $\mathrm{i}=1,3$ \\
$\{\mathbf{x}\}$ & system vector containing unknowns \\
$x_{i}$ & the Kronecker delta \\
\hline &
\end{tabular}




\begin{tabular}{ll}
$\omega_{i}$ & weight functions of the Gaussian points \\
$\psi$ & solid angle at a point \\
$\sigma_{i}$ & principal stresses, $\mathrm{i}=1,3$ \\
$\sigma_{i j}$ & stress tensor, $\mathrm{i}, \mathrm{j}=1,3$ \\
$\xi_{i}$ & intrinsic coordinates of elements, $\mathrm{i}=1,2$ \\
\hline
\end{tabular}




\section{Chapter 1}

\section{Introduction}

The Boundary Element Method (BEM) is a more recently developed numerical method for engineering analysis. The characteristic feature of this computational technique is that only the boundary or the surface of the solution domain needs to be discretized, unlike the commonly used finite element method (FEM). The BEM has now been well established as a very efficient computational tool for solving a large class of problems, in particular, those involving rapidly varying solution variables. In elastostatics, such problems occur in solids with stress concentrations and cracks. The BEM is based on the boundary integral equation (BIE) that gives its characteristic feature as a boundary solution technique. The BIE is derived using the fundamental solutions to the governing differential equations (also known as the Green's functions) and the reciprocal work theorem. These fundamental solutions, which form the kernel functions of the BIE, are singular with respect to the distance between the source (or load) point and the field point of the solution domain. In the development of the BEM over the years, numerous efforts have been made in the literature to eliminate or "regularize" the singular integrals to allow accurate numerical evaluation of these integrals. They include analytical as well as

numerical procedures. The analytical approaches are, generally, relatively complex mathematically, while the numerical approaches tend to involve algorithms that are 
relatively cumbersome to implement or are only suitable for specific applications.

A "self-regularization" technique was proposed by Cruse and Richardson (1996) in a BEM formulation for the evaluation of the displacements and stresses at interior points very close to the boundary of the domain in two-dimensional (2D) elastostatics, via Somigliana's displacement and stress identities, respectively. A near-singularity condition in the integrals arises in this case as the interior point gets closer and closer to the boundary. This makes the accurate numerical evaluation of the integrals over the boundary elements close to these interior points a very serious issue. The self-regularization technique they had proposed overcomes this difficulty with relative ease, as it is mathematically quite straightforward and its implementation into existing BEM codes is also relatively simple. The usefulness of such a scheme has been demonstrated by Yu (2006), Shah et al. (2006) and Shah (2007) for obtaining interior point solutions required in the determination of the T-stress in fracture mechanics studies. In this study, this technique is extended to the BEM analysis of three-dimensional (3D) bodies by modifying an existing BEM code based on the quadratic isoparametric element formulation. It will be explained in Chapter 3 and its successful implementation demonstrated by some examples.

The Somigliana's stress identity was extended using this technique to the boundary in Richardson and Cruse (1999) to form the self-regularized traction-BIE for boundary solutions. A requirement for convergence of the numerical solution in the BEM implementation is displacement-gradient (or $C^{1, \alpha}$ ) continuity between the boundary elements at the inter-element nodes. These authors showed that despite using conforming $C^{0}$ elements, but employing a "relaxed continuity" approach, good results could still be obtained. They do, however, recommend the use of higher order conforming elements beyond the quadratic variation. It should be noted that 
the quadratic isoparametric element formulation is the most commonly used one in BEM analysis.

Further reports by Martin et al. (1998) and Ribeiro et al. (2002) show that this self-regularized traction-BIE scheme could not always give good results unless higher order or non-conforming elements are used. The use of non-conforming elements have been used before in BEM analysis, see, e.g. Dominguez and Ariza (2000), where collocation is carried out at points inside the elements instead of at the nodes associated with the elements, thereby ensuring the satisfaction of the continuity requirements. Both these "relaxed continuity" approach of Richardson and Cruse (1999) and the use of non-conforming elements in the traction-BIE have been tested only in 2D or part of a 3D BEM analysis of elastic bodies, and in potential theory, and show promising results. To the author's knowledge, there are no reports of applying this self-regularization BEM scheme for the analysis of three dimensional elastic solids. Another focus of this thesis is thus to extend the scheme wholly with the approaches tried in $2 \mathrm{D}$, to 3D elastostatics.

Another class of problems involving near-singularities in the integrals deals with thin, slender domains, commonly referred to as the thin-body problem. Examples of such problems are thin aerofoils and turbine blades as well as thin laminates. It is suggested in Richardson and Cruse (1999) that the self-regularized traction-BIE mentioned above, can provide a suitable solution tool for such problems as well. It will be shown later in Chapter 4 , however, that this is not the case for the reason which will be explained then. A self-regularized displacement-BIE, similar to the approaches in Chapters 3 and 4 , is proposed instead in Chapter 5 for the 3D BEM, specifically to treat the thin-body problem. It has not been introduced previously in the literature. 
First, however, it is useful to provide a brief review of the BEM for $3 \mathrm{D}$ elastostatics based on the conventional displacement-BIE. This is presented in the next chapter. The basic analytical formulations and the numerical implementation will be described. The near-singularity issue arising in problems requiring interior point solutions very close to the surface of the domain and when treating thin-body problems will also be discussed. 


\section{Chapter 2}

\section{Review of Boundary Element Method}

\subsection{Introduction}

The boundary element method (BEM) is an alternative numerical method to, e.g., the conventional finite element method which has been applied to solve problems in many branches of engineering. It has been established as an efficient and accurate tool especially when treating certain types of problems, such as those involving cracks and stress concentrations in elastostatics. This chapter introduces the basic concepts of $\mathrm{BEM}$, and describes the boundary integral equations (BIE) that form the analytical basis of the BEM. Some problems that arise due to the singularities of the kernel functions in the BIE are discussed, as well as possible strategies to solve them. The traction-BIE is also introduced as an alternative boundary integral equation to the conventionally used displacement-BIE; it is typically used for treating crack problems but has gained attraction beyond applications to fracture mechanics. 


\subsection{The Boundary Element Method in}

\section{Elastostatics}

In the attempts to determine the stress distribution and deformation of solid bodies, pressure distribution over a surface in an incoming fluid flow, or temperature and flux distribution within a body under the influence of a heat source, numerical methods are often employed. This can help reduce the time and cost considerably in the design and performing experiments where required. It can also help improve the design of engineering components before they are manufactured. In order to serve this purpose, several numerical methods have been developed since the 1940's and are widely used in various fields in engineering. Among these techniques, the boundary element method (BEM) is a more recently-developed method. It has been proven to be an efficient and accurate numerical method when compared with other conventional methods such as the finite element method (FEM), the finite difference method (FDM), and the finite volume method (FVM).

In solid mechanics, the geometry and the displacements are continuous all over the domain of a problem. In order to numerically analyze the problem, the domain often needs to be divided into a mesh of small elements, over each of which the geometry, tractions and displacements are represented by certain polynomials in terms of intrinsic coordinates, which are related to global coordinates by certain relationships. In domain-solution method, i.e. FEM, FDM and FVM, both the

surface and interior of the domain are discretized. In BEM, however, only the surface is discretized, leaving the interior continuous. This reduces the dimensional order of elements that are used by one, i.e., line elements are used for two-dimensional problems, and surface elements are used for three-dimensional problems. Typical FEM and BEM meshes used for two- and three-dimensional problems are shown in 
Figure 2.1.

Since the interior of the domain remains continuous in BEM, less of the domain is discretized or approximated compared to domain-solution methods. The results will also be more accurate because of the use of singular fundamental solutions. Thus, one can afford to use fewer number of elements than in other methods for a particular model. This will become more evident when calculating stresses and displacements at an interior point inside the domain; they can be directly obtained from known boundary values in BEM as a secondary procedure, while in FEM they need to be interpolated by the element that contains the interior point if it is not exactly on an interior node. All these features thus significantly reduce the number of elements and nodes on which tractions and displacements are to be calculated, thus reducing the amount of numerical modeling work as well as computational time. It also makes BEM more suited for preliminary design since a surface mesh is easy to modify locally when the outer geometry is changed.

Both BEM and FEM calculate nodal values by forming a system matrix which is to be inverted to get the unknowns. In FEM the matrix is symmetric and sparsely populated. In BEM, however, the matrix is unsymmetric and fully populated with non-zero coefficients. However, it should be noted that the size of the matrix is very much smaller than that in FEM, thus this disadvantage of fully-populated matrix is more than compensated for by the smaller amount of matrix coefficients that need to be stored. A severe drawback of BEM is that it is better suited for problems with a relatively small surface/volume ratio, but not for thin body problems where the distance between two nodal points can be small. This is due to the singularity of the kernel functions in the boundary integral equations, which will be evident later below. Also when an interior point is close to the boundary, the results will 
again suffer because of the near singularities of the integrands. The FEM therefore is generally more efficient in treating thin-body problems. These issues are the focus of this study and will be treated in the chapters that follow.

\subsection{Boundary Integral Equations}

The well-known displacement-boundary integral equation (displacement-BIE) is derived from Betti's reciprocal work theorem and the use of the fundamental solution to the Kelvin's problem in elasticity. The process of this derivation is described in the literature, see, e.g., Tan (1987), Becker (1992) and Kane (1994). The displacement-BIE can be expressed as:

$$
C_{i j}(P) u_{j}(P)+\int_{S} T_{i j}(P, Q) u_{j}(Q) d S(Q)=\int_{S} U_{i j}(P, Q) t_{j}(Q) d S(Q)
$$

where $P$ and $Q$ are the source point and field point, respectively, and the free-term $C_{i j}$ is a constant, second-order tensor whose values depend on the geometry of the boundary at $P$. Also, $u_{i}(Q)$ and $t_{i}(Q)$ are the displacements and tractions at the boundary point $Q$, respectively. $U_{j i}$ and $T_{j i}$ in Equation (2.1) are the kernel functions corresponding to the Kelvin's fundamental solutions for displacements and tractions, respectively. They represent the displacements and tractions, respectively, in the $i$-th direction due to a point load at $P$ in the $j$-th direction. In three dimensions, they are expressed as

$$
U_{i j}=\frac{1}{16 \pi \mu(1-\nu) r}\left[(3-4 \nu) \delta_{i j}+r_{, i} r_{, j}\right]
$$

and

$$
T_{i j}=\frac{-1}{8 \pi(1-\nu) r^{2}}\left\{\frac{\partial r}{\partial n}\left[(1-2 \nu) \delta_{i j}+3 r_{, i} r_{, j}\right]-(1-2 \nu)\left(n_{j} r_{, i}-n_{i} r_{, j}\right)\right\}
$$


where $r$ is the distance between $P$ and $Q$, and $n_{i}$ is the unit outward normal at $Q$. The comma index denotes derivative with respect to coordinates $x_{i}$, i.e. $r_{, i}=\partial r / \partial x_{i}$, and the normal derivative is expressed by

$$
\frac{\partial r}{\partial n}=\frac{\partial r}{\partial x_{i}} \frac{\partial x_{i}}{\partial n}
$$

The displacements and tractions are the primary solution unknowns that need to be computed in order to solve an elasticity problem. For a particular load case, either the displacement or traction is known in one direction on a part of the surface, with the other quantity remaining unknown. The surface $S$ of the domain is generally divided into two categories: one with displacements prescribed, denoted by $S_{u}$, the other with tractions prescribed, denoted by $S_{t}$, as shown in Figure 2.2.

\subsection{Numerical Integration}

The analytical solution to Equation (2.1) is usually not possible to obtain for arbitrary geometries. Numerical methods must therefore be employed. In order to do this, the surface of the solid is divided into a mesh of small elements. The integration in Equation (2.1) is replaced by the summation of integrals over each of the elements.

As mentioned earlier, line elements are used for two-dimensional problems and surface elements are used in three dimensions. Over each element, the variations of both the geometry and field variables (displacements and tractions) are assumed; they can be constant, linear, quadratic, cubic or higher order. The geometry and the field variables can be described in different orders as well. However, based on experience in both FEM and BEM, quadratic variation for both geometry and field 
variables has been shown to be very efficient while providing good accuracy. Elements using the same variation for geometry and field variables are called isoparametric elements. Thus, in boundary element analysis, the most commonly used elements are isoparametric quadratic elements having quadrilateral or triangular shapes with either eight or six nodes at the corners and mid-points of the sides. Each element is defined by a set of intrinsic coordinates $\xi_{1}$ and $\xi_{2}$ varing from -1 to 1 for quadrilateral element and 0 to 1 for triangular element, as shown in Figure 2.3. The global coordinates of a point lying on an element are mapped by a set of shape functions $N_{c}\left(\xi_{1}, \xi_{1}\right)$ and global coordinates of the nodes as follows:

$$
x_{i}\left(\xi_{1}, \xi_{2}\right)=\sum_{c=1}^{6} N_{c}\left(\xi_{1}, \xi_{2}\right) x_{i}^{c}
$$

Since the element is isoparametric, displacements and tractions are expressed in the same way:

$$
u_{i}\left(\xi_{1}, \xi_{2}\right)=\sum_{c=1}^{6 \text { or } 8} N_{c}\left(\xi_{1}, \xi_{2}\right) u_{i}^{c}
$$

and

$$
t_{i}\left(\xi_{1}, \xi_{2}\right)=\sum_{c=1}^{6} N_{c}\left(\xi_{1}, \xi_{2}\right) t_{i}^{c}
$$


For quadrilateral elements, the shape functions are:

$$
\begin{aligned}
& N_{1}\left(\xi_{1}, \xi_{2}\right)=\frac{-1}{4}\left(1-\xi_{1}\right)\left(1-\xi_{2}\right)\left(1+\xi_{1}+\xi_{2}\right) \\
& N_{2}\left(\xi_{1}, \xi_{2}\right)=\frac{1}{2}\left(1-\xi_{1}^{2}\right)\left(1-\xi_{2}\right) \\
& N_{3}\left(\xi_{1}, \xi_{2}\right)=\frac{-1}{4}\left(1+\xi_{1}\right)\left(1-\xi_{2}\right)\left(1-\xi_{1}+\xi_{2}\right) \\
& N_{4}\left(\xi_{1}, \xi_{2}\right)=\frac{1}{2}\left(1+\xi_{1}\right)\left(1-\xi_{2}^{2}\right) \\
& N_{5}\left(\xi_{1}, \xi_{2}\right)=\frac{-1}{4}\left(1+\xi_{1}\right)\left(1+\xi_{2}\right)\left(1-\xi_{1}-\xi_{2}\right) \\
& N_{6}\left(\xi_{1}, \xi_{2}\right)=\frac{1}{2}\left(1-\xi_{1}^{2}\right)\left(1+\xi_{2}\right) \\
& N_{7}\left(\xi_{1}, \xi_{2}\right)=\frac{-1}{4}\left(1-\xi_{1}\right)\left(1+\xi_{2}\right)\left(1+\xi_{1}-\xi_{2}\right) \\
& N_{8}\left(\xi_{1}, \xi_{2}\right)=\frac{1}{2}\left(1-\xi_{1}\right)\left(1-\xi_{2}^{2}\right)
\end{aligned}
$$

The shape functions for triangular elements are:

$$
\begin{aligned}
& N_{1}\left(\xi_{1}, \xi_{2}\right)=\left(1-\xi_{1}-\xi_{2}\right)\left(2\left(1-\xi_{1}-\xi_{2}\right)-1\right) \\
& N_{2}\left(\xi_{1}, \xi_{2}\right)=4 \xi_{1}\left(1-\xi_{1}-\xi_{2}\right) \\
& N_{3}\left(\xi_{1}, \xi_{2}\right)=\xi_{1}\left(2 \xi_{1}-1\right) \\
& N_{4}\left(\xi_{1}, \xi_{2}\right)=4 \xi_{1} \xi_{2} \\
& N_{5}\left(\xi_{1}, \xi_{2}\right)=\xi_{2}\left(2 \xi_{2}-1\right) \\
& N_{6}\left(\xi_{1}, \xi_{2}\right)=4 \xi_{2}\left(1-\xi_{1}-\xi_{2}\right)
\end{aligned}
$$

The shape function $N_{c}\left(\xi_{1}, \xi_{2}\right)$ of node $c$ equals to 1 at node $c$, and 0 at all other nodes. The coordinates and field variables in the global coordinate system at a point within the element are now explicitly expressed by the nodal values. 
In order to integrate over the element, Gaussian quadrature is used for quadrilateral elements while the Hammer-Stroud formula is used for triangular elements. Both integration schemes select several integration points on the element whose intrinsic coordinates are pre-determined by a series of known abscissae, at which the values are calculated. They are then multiplied by a weight function, and summed to obtain the approximate value of the integral, as follows (Becker 1992):

$$
\int_{-1}^{1} \int_{-1}^{1} f\left(\xi_{1}, \xi_{2}\right) d \xi_{1} d \xi_{2} \approx \sum_{m=1}^{G_{m}} \sum_{n=1}^{G_{n}} f\left(\xi_{1}, \xi_{2}\right) \omega_{n} \omega_{m}
$$

for Gaussian quadrature, and

$$
\int_{0}^{1} \int_{0}^{1} f\left(\xi_{1}, \xi_{2}\right) d \xi_{1} d \xi_{2} \approx \sum_{i=1}^{N} f\left(\xi_{1}, \xi_{2}\right) \omega_{i}
$$

for triangular elements, where $G_{m}$ and $G_{n}$ are the number of Gaussian points in the two directions, which are usually set to be identical; $N$ the number of Hammer-Stroud integration points; and $\omega_{i}$ the weight functions. A large number of integration points provides better accuracy, but at the expense of computing time. Therefore a balance between accuracy and efficiency needs to be found. In the computer code used in this study, as used in Tan and Fenner (1978), $4 \times 4\left(G_{m}=G_{n}=4\right)$ integration points for quadrilateral elements and 13 points for triangular elements are employed for most cases. They provide very good results while taking relatively low computing time.

A transformation from the elemental coordinates $\xi_{1}, \xi_{2}$ to the global coordinates $x_{i}$ is needed, i.e.

$$
d S(Q)=J\left(\xi_{1}, \xi_{2}\right) d \xi_{1} d \xi_{2}
$$


where $J\left(\xi_{1}, \xi_{2}\right)$ is the Jacobian of transformation. It may be expressed as

$$
\begin{aligned}
J\left(\xi_{1}, \xi_{2}\right) & =\frac{\partial S}{\partial\left(\xi_{1}, \xi_{2}\right)}=\sqrt{J_{1}^{2}+J_{2}^{2}+J_{3}^{2}} \\
& =\sqrt{\left(\frac{\partial x_{2}}{\partial \xi_{1}} \frac{\partial x_{3}}{\partial \xi_{2}}-\frac{\partial x_{3}}{\partial \xi_{1}} \frac{\partial x_{2}}{\partial \xi_{2}}\right)^{2}+\left(\frac{\partial x_{3}}{\partial \xi_{1}} \frac{\partial x_{1}}{\partial \xi_{2}}-\frac{\partial x_{1}}{\partial \xi_{1}} \frac{\partial x_{3}}{\partial \xi_{2}}\right)^{2}+\left(\frac{\partial x_{1}}{\partial \xi_{1}} \frac{\partial x_{2}}{\partial \xi_{2}}-\frac{\partial x_{2}}{\partial \xi_{1}} \frac{\partial x_{1}}{\partial \xi_{2}}\right)^{2}}
\end{aligned}
$$

where

$$
\frac{\partial x_{i}}{\partial \xi_{j}}=\sum_{c=1}^{6} \frac{\partial N_{c}\left(\xi_{1}, \xi_{2}\right)}{\partial \xi_{j}} x_{i}^{c}
$$

is the derivative of global coordinate $x_{i}$ with respect to intrinsic coordinate $\xi_{j}$. The unit outward normal, $n_{i}$, is then expressed by:

$$
n_{i}=\frac{J_{i}}{\left|J\left(\xi_{1}, \xi_{2}\right)\right|}
$$

When $P$ is a node of a quadrilateral element, integrating over the integrand with $T_{i j}$ in Equation (2.3) is singular and varies very rapidly. Thus a somewhat different integration scheme has to be adopted. This was done by dividing quadrilateral elements into either two or three triangular sub-elements depending on whether the node is a corner node or mid-side node. A set of local system of coordinates and linear shape functions are introduced to transfer coordinates from the sub-element to the parent element (Lachat and Watson 1976) as follows:

$$
\xi_{\alpha}(\eta)=\frac{1}{4}\left(1+\eta_{1} \eta_{1}^{m}\right)\left(1+\eta_{2} \eta_{2}^{m}\right) \xi_{\alpha}^{(m)}, \quad m=1,4
$$

In Equation (2.16), $\eta_{\alpha}^{m}$ are the intrinsic triangular coordinates of the $m$ th node of the sub-element, and $\xi_{\alpha}^{(m)}$ are the intrinsic coordinates of the vertices of the triangular sub-element. At the vertex where $P$ is located, $\xi_{\alpha}^{(1)}=\xi_{\alpha}^{(2)}$. The Jacobian from the sub-elemental coordinate system $\eta_{\alpha}$ to the elemental coordinate system $\xi_{\alpha}$ is then 
expressed as:

$$
\begin{aligned}
\bar{J}\left(\eta_{1}, \eta_{2}\right) & =\frac{\partial\left(\xi_{1}, \xi_{2}\right)}{\partial\left(\eta_{1}, \eta_{2}\right)} \\
& =\frac{\partial \xi_{1}\left(\eta_{1}, \eta_{2}\right)}{\partial \eta_{1}} \frac{\xi_{2}\left(\eta_{1}, \eta_{2}\right)}{\partial \eta_{2}}-\frac{\xi_{2}\left(\eta_{1}, \eta_{2}\right)}{\partial \eta_{1}} \frac{\partial \xi_{1}\left(\eta_{1}, \eta_{2}\right)}{\partial \eta_{2}}
\end{aligned}
$$

Then the integral over a quadrilateral element containing source point $P$ becomes

$$
\begin{aligned}
\int_{-1}^{1} \int_{-1}^{1} f(P, Q) d S(Q) & =\int_{-1}^{1} \int_{-1}^{1} f\left(\xi_{1}, \xi_{2}\right) J\left(\xi_{1}, \xi_{2}\right) d \xi_{1} d \xi_{2} \\
& =\int_{-1}^{1} \int_{-1}^{1} f\left(\eta_{1}, \eta_{2}\right) J\left(\xi_{1}, \xi_{2}\right) \bar{J}\left(\eta_{1}, \eta_{2}\right) d \eta_{1} d \eta_{2}
\end{aligned}
$$

Substituting Equations (2.6),(2.7),(2.10) and (2.12) into Equation (2.1), the following expression is obtained for a model meshed using $n$ nodes and $M$ elements:

$$
\begin{aligned}
C_{i j}\left(P^{a}\right) u_{j}\left(P^{a}\right) & +\sum_{b=1}^{M} \sum_{c=1}^{8 \text { or } 6} u_{j}\left(P^{d(b, c)}\right) \int_{S_{b}} T_{j i}\left(P^{a}, Q\left(\xi_{1}, \xi_{2}\right)\right) N^{c}\left(\xi_{1}, \xi_{2}\right)\left|J\left(\xi_{1}, \xi_{2}\right)\right| d \xi_{1} d \xi_{2} \\
& =\sum_{b=1}^{M} \sum_{c=1}^{8 \text { or } 6} t_{j}\left(P^{d(b, c)}\right) \int_{S_{b}} U_{i j}\left(P^{a}, Q\left(\xi_{1}, \xi_{2}\right)\right) N^{c}\left(\xi_{1}, \xi_{2}\right)\left|J\left(\xi_{1}, \xi_{2}\right)\right| d \xi_{1} d \xi_{2}
\end{aligned}
$$

where $P^{a}$ is the node number ranging from 1 to $n, b$ is the element number ranging from 1 to $M$, and $P^{d(b, c)}$ denotes the $c$-th node of the $b$-th element. The displacements and tractions at the Gaussian points are interpolated by nodal values of $u_{i}(P)$ and $t_{i}(P)$ using the shape functions, which are fixed for an element so they are taken outside the integration. Note that the nodal displacements are the same on different elements sharing the node, but tractions can vary from element to element according to the boundary conditions imposed on that element. 
There was no simple explicit formula for the $C_{i j}$ terms in 3-D until very recently, see, e.g., Dangla et al. (2005). The approach proposed in this paper is to analytically calculate the $C_{i j}$ terms based on the local geometry of the node. However, an indirect approach is widely practised in the boundary element community as generally there is no need to obtain these values analytically. This indirect approach uses the rigid-body motion condition, where the tractions $t_{j}(P)$ on the right-hand side of Equation (2.19) are all zero. The diagonal sub-matrix can then be obtained by:

$$
\begin{aligned}
& C_{i j}\left(P^{a}\right)+\sum_{b=1}^{M} \sum_{c=1}^{8 \text { or } 6} \delta_{a d} \int_{S_{b}} T_{j i}\left(P^{a}, Q\left(\xi_{1}, \xi_{2}\right)\right) N^{c}\left(\xi_{1}, \xi_{2}\right)\left|J\left(\xi_{1}, \xi_{2}\right)\right| d \xi_{1} d \xi_{2} \\
& =-\sum_{b=1}^{M} \sum_{c=1}^{8 \text { or } 6}\left(1-\delta_{a d}\right) \int_{S_{b}} T_{j i}\left(P^{a}, Q\left(\xi_{1}, \xi_{2}\right)\right) N^{c}\left(\xi_{1}, \xi_{2}\right)\left|J\left(\xi_{1}, \xi_{2}\right)\right| d \xi_{1} d \xi_{2}
\end{aligned}
$$

Equation (2.19) represents a set of $3 n$ equations. It can be expressed in matrix form as:

$$
[\mathbf{A}]\{\mathbf{u}\}=[\mathbf{B}]\{\mathbf{t}\}
$$

where $[\mathbf{A}]$ and $[\mathbf{B}]$ are matrices storing coefficients of displacements and tractions, respectively, and $\{\mathbf{u}\}$ and $\{\mathbf{t}\}$ are vectors that contain displacements and tractions at all the nodes.

The boundary conditions imposed on the boundaries are transfered to the elements, with either prescribed displacements or tractions. In some cases, nodal constraints are also applied when displacement constraints on the surface are not present to prevent rigid body motion. Therefore at each boundary node, in each direction, either the displacement or the traction is known. Whenever there is an 
known value, it is multiplied by the corresponding coefficient and put into a second member $\{\mathbf{b}\}$, and the coefficient of the unknown will remain in matrix $[\overline{\mathbf{A}}]$. After this step, Equation (2.21) becomes

$$
[\overline{\mathbf{A}}]\{\mathbf{x}\}=\{\mathbf{b}\}
$$

where vector $\{\mathbf{x}\}$ stores all the unknown displacements or tractions. Now the set of equations are ready to be solved by, say Gauss elimination. After solving for $\{\mathbf{x}\}$, the displacements and tractions at all boundary nodes are known. The boundary stresses are obtained from the displacements and tractions at each node. This is well-established in literature and will not be repeated here.

\subsection{Stress and Displacement Solution for Interior Points}

After solving the system equations in Equation (2.22), the displacements and stresses at the boundary nodes are known. The displacements $u_{i}(p)$ and stresses $\sigma_{i j}(p)$ at an interior point $p$ can now be obtained, if required, using the calculated values of the displacements and tractions at the boundary nodes via Somigliana identities:

$$
u_{j}(p)=-\int_{S} u_{i}(Q) T_{j i}(p, Q) d S(Q)+\int_{S} t_{i}(Q) U_{j i}(p, Q) d S(Q)
$$

and

$$
\sigma_{i j}(p)=-\int_{S} u_{k}(Q) S_{k i j}(p, Q) d S(Q)+\int_{S} t_{k}(Q) D_{k i j}(p, Q) d S(Q)
$$


where $U_{i j}$ and $T_{i j}$ are defined before and $S_{k i j}(p, Q)$ and $D_{k i j}(p, Q)$ are third-order tensors expressed as:

$$
\begin{aligned}
S_{k i j}(p, Q) & =\frac{\mu}{4 \pi(1-\nu) r^{3}}\left\{3 \frac{\partial r}{\partial n}\left[(1-2 \nu) \delta_{i j} r_{, k}+\nu\left(\delta_{k i} r_{, j}+\delta_{k j} r_{, i}\right)-5 r_{, i} r_{, j} r_{, k}\right]\right. \\
& +n_{i}\left[3 \nu r_{, j} r_{, k}+(1-2 \nu) \delta_{k j}\right] \\
& +n_{j}\left[3 \nu r_{, i} r_{, k}+(1-2 \nu) \delta_{k i}\right] \\
& \left.+n_{k}\left[3(1-2 \nu) r_{, i} r_{, j}-(1-4 \nu) \delta_{i j}\right]\right\}
\end{aligned}
$$

and

$$
\begin{aligned}
D_{k i j}(p, Q)= & \frac{1-2 \nu}{8 \pi(1-\nu) r^{2}}\left[\delta_{k i} r_{, j}+\delta_{k j} r_{, i}\right. \\
& \left.-\delta_{i j} r_{, k}+\frac{3}{1-2 \nu} r_{, i} r_{, j} r_{, k}\right]
\end{aligned}
$$

These third-order tensors are obtained from the derivative form of Equation (2.23) and using Hooke's Law. The integrals are evaluated by Gaussian quadrature on each element as before.

Interior point solutions are typically even more accurate than boundary solutions since the interior of the domain is not discretized or approximated. However, when the interior point is close to the boundary, due to the so-called near-singularities of the kernels $S_{k i j}$ and $D_{k i j}$ which are of order of $r^{-3}$ and $r^{-2}$, respectively, the integrands over the element close to the interior point will vary very rapidly. This can cause the results to deteriorate, especially the stresses, if a fixed quadrature scheme is employed. This is known as the "boundary layer effect" in the BEM 
literature. A direct way of solving this problem is to refine the surface mesh near the interior point, or to significantly increase the number of Gauss points. As can be seen in Li (2003), however, this method often requires a very refined mesh, which is impractical when multiple interior points are needed, and even more cumbersome in three dimensions. A similar approach is seen in Jun et al. (1985) where a simple partition of element close to the point is used.

Other methods of removing the singularity are by "regularization", such as by analytically integrating over each element close to the interior point. For example, Granados and Gallego (2001) used regularization in a complex plane, and Niu et al. (2005) proposed a semi-analytical approach to transfer the singular surface integral into a series of line integrals by using integration by parts. Other analytical approaches can be seen in Ma and Kamiya (2001), Zhou et al. (2008), and Xie et al. (2011). These approaches can provide very accurate results in two dimensions, but are either analytically and/or numerically, very complex and difficult to apply to general three-dimensional problems. A very simple approach was proposed by Cruse and Richardson (1996) in which simple solutions of a rigid body motion and constant stress state are added and subtracted back into Equations (2.23) and (2.24), respectively. Detailed analysis of this scheme can be seen in Richardson (1998). Compared to other approaches, this method is relatively simple both analytically and numerically. This method was implemented for two-dimensional elastostatics in Cruse and Richardson (1996), Richardson and Cruse (1999). A similar approach was employed for potential theory by Sladek et al. (1993). It has also been employed in the evaluation of T-stress in fracture mechanics where interior points on the integration path can be located close to the crack surface or interface between two subregions (Yu 2006, Shah et al. 2006, Shah 2007). No implementation for three-dimensional problems, however, has been reported to the author's knowledge. 
It will be extended to three dimensions in this study, and will be presented in Chapter 3.

\subsection{Traction-BIE and Hypersingular-BIE}

Besides the conventional displacement-BIE, another category of boundary integral equations is sometimes also used in BEM, known as the traction-BIE. It is often used in conjunction with displacement-BIE for crack problems, as in the dual boundary element method, see e.g., Mi and Aliabadi (1992). It greatly simplifies the discretization process as well as improving the accuracy (Dominguez and Ariza 2000).

Traction-BIE is formed by differentiating conventional displacement-BIE with respect to the coordinates and multiplying unit outward normals at the source point. For $P$ lying on a smooth surface, the following equation (Jin et al. 2011) is obtained:

$$
\frac{1}{2} t_{i}(P)+n_{j}(P) \int_{S} S_{k i j}(P, Q) u_{k}(Q) d S(Q)+n_{j}(P) \int_{S} D_{k i j}(P, Q) t_{k}(Q) d S(Q)
$$

where $n_{i}(P)$ is the unit outward normal at source point $P$. The third-order tensors $S_{k i j}$ and $D_{k i j}$ are given in Equations (2.25) and (2.26); they are of order of $r^{-3}$ and $r^{-2}$ respectively. They are hypersingular, which imposes a difficulty to the numerical method if standard integration is to be used. This is especially so when the source point belongs to the element being integrated over. The sub-division of element as in conventional BIE will not work since the singularity of the kernels in traction-BIE are one order higher than that in displacement-BIE; this process only yields order $r$ as $P \rightarrow Q$ 
A common approach to reduce the singularities of the integrands of the integrals in Equation (2.27) is to analytically compute the singular integrals, such as in Chien et al. (1991) where superposition of rigid body motion was implemented, thereby leading to the need to evaluate integrals in Cauchy principal value sense. The idea of subtracting and adding back terms of known stress state or terms in Taylor series from the density function to reduce the singularities has also been used in several papers. Rudolphi (1991) first introduced the use of simple solutions to regularize hypersingular integrals; Cruse and Suwito (1993) subtracted and added back terms which were then analytically computed; Liu and Rizzo (1992), Li and Huang (2010) applied this idea in three-dimensional acoustic problems. In Richardson and Cruse (1999), the self-regularization for interior point is extended to traction-BIE by taking the interior point to the boundary in Somigliana identity for stresses. It follows the similar process as that for interior point, and no analytical solution is involved. This method is thus both analytically and numerically simple, and is easy to be implemented in an existing computer code. This will be discussed in detail in Chapter 4 .

Another difficulty of the hypersingular-BIE is the requirement of displacementgradients or stress continuity on the boundary, known as the $C^{1, \alpha}$ Holder continuity. A detailed discussion of the continuity requirement can be seen in Martin and Rizzo (1996). In conventional BIE, the most commonly used quadratic elements are only $C^{0, \alpha}$ continuous, i.e. displacement are continuous all over the boundary, but displacement derivatives and stresses are discontinuous across the element boundary. Several papers deal with this problem, mostly in two dimensions, however. Jin et al. (2011) used an adaptive meshing scheme to make sure that the source point is always the mid-point of an element where stresses are continuous; Wang and $\mathrm{Li}$ (2009) developed a set of novel shape functions to relate nodes on different elements sharing the source point to ensure continuity of displacement derivatives 
at the source point; Jorge et al. (2003) added extra equations to the system matrix to enforce displacement derivatives to be the same on different elements sharing the source point. These methods are efficient and accurate in 2-D, but are not as straightforward to implement into 3-D. For example, in 2-D every extreme node is shared by two elements, while in 3-D, only the mid-point nodes are shared by two elements, but the corner nodes are shared by at least three elements, usually four or more. The idea by Jin et al. (2011) thus cannot be easily extended to 3-D in a general way. Due to the same reason, the shape functions developed in Wang and Li (2009) will be much more complex in 3-D. In the continuity enforcement strategy, for a node shared by $m$ elements, $m$ set of extra equations are to be written to be placed in the system matrix. Even for a simple block with one element on each face with a total of 20 nodes, hundreds of extra equation will be added to the matrix while the original system matrix is only $60 \times 60$.

Based on their earlier analysis in Cruse and Richardson (1996), Richardson and Cruse (1999) proposed a "relaxed continuity" strategy to approximate continuity across element boundaries. This scheme takes the average of the displacement derivatives at a node according to the element it belongs to. They showed some results of simple two-dimensional elasticity problems, but suggested that higher order elements are still preferable to using quadratic elements. This issue was also discussed in detail in Martin et al. (1998). However, Ribeiro et al. (2002) have argued that this relaxed continuity approach is the main source of error when using conforming elements. They showed that the use of non-conforming elements, whose collocation points are located inside the element instead of at the boundary nodes, provides better results just as well as with the use of higher-order elements. The use of both non-conforming elements, which increases the size of the system matrix since collocations are done at interior of the elements, and higher-order elements, will 
increase computing time significantly particularly in three dimensions. A strategy similar to the use of non-conforming element but with the same size of system matrix as in displacement-BIE, was used in two-dimensional elastodynamics by Gallego and Dominguez (1996). Ribeiro et al. (2009) implemented this scheme for two-dimensional potential problems, and 3-D acoustic problems were treated in Liu and Chen (1999) using the scheme. Dominguez and Ariza (2000) and Dominguez et al. (2000) adopted this strategy for three-dimensional fracture problem where traction-BIE is only applied to elements on the crack surface while for the other elements in the problem, the displacement-BIE is applied. In this study, the self-regularization scheme is adopted, along with the collocation strategy applied to the entire surface of the domain. The relaxed continuity will also be tested and discussed in Chapter 4.

As mentioned previously, a class of problems that BEM is not very well suited for are those that involve thin bodies. In these problems, the distance between source point and field point, $r$, can be small when two surfaces are close to each other, making the kernel functions vary rapidly over the elements. This leads to errors in numerical integration if a fixed quadrature scheme is employed. The methods to solve this problem in the literature are, again, mostly to analytically integrate the singular kernels, see, e.g., Ye and Liu (1985), Liu (1987), and Krishnasamy et al. (1994). Liu (1998) proposed a non-degeneracy approach by adding and subtracting a term to the conventional displacement-BIE which was analytically calculated by line-integrals using Stokes' theorem, and obtained good results. Cruse and Aithal (1993) also proposed an approach to solve thin-body problems by the use of regularization of integral operators and a theta integration algorithm. All these analytical approaches are, again, mathematically complex and not that simple to implement. It was suggested in Richardson and Cruse (1999) that the self-regularized traction-BIE 
should be well-suited for thin-body problems. The self-regularized traction-BIE is also a focus of this thesis. It will be dealt with in greater detail in Chapter 4 .

\subsection{Concluding Remarks}

This Chapter has reviewed the concepts of boundary element method and its advantages over some other numerical methods. The steps in the derivation of the displacement-BIE were presented. How interior point solutions can be obtained as a secondary procedure has also be described. The traction-BIE was then introduced as an alternative boundary integral equation. Two main difficulties due to the singularity of the kernel functions were discussed. They are, (a) the loss of accuracy of the results of displacement and stress at the interior points close to boundary, and (b) hypersingularity of the integrals involved in the traction-BIE. These two problems are the main topics of the next two chapters. 


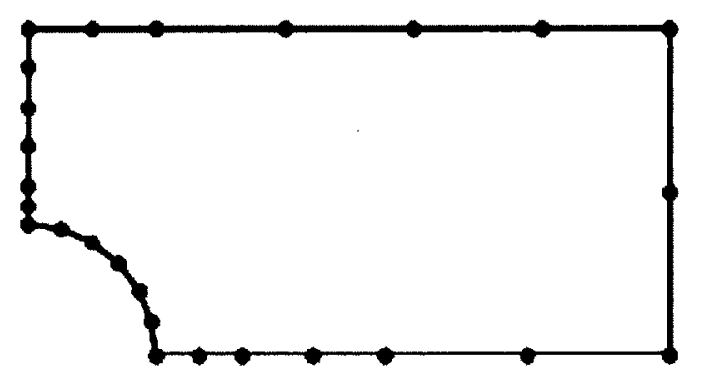

a) BEM mesh for $2 D$ problem, 13 element, 26 nodes

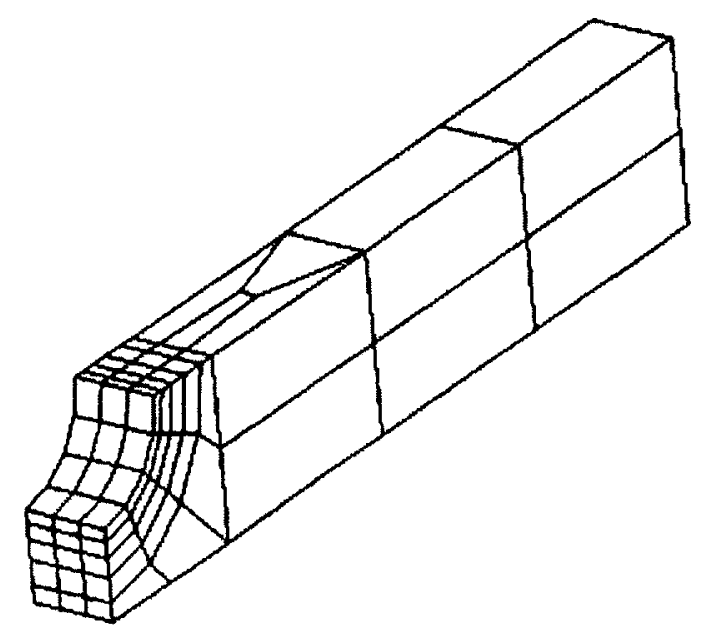

c) BEM mesh for 3D problem, 114 element, 344 nodes

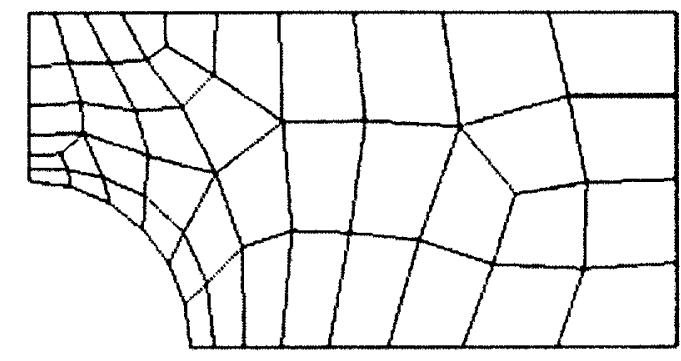

b) FEM for 2D problem, 39 elements, 146 nodes

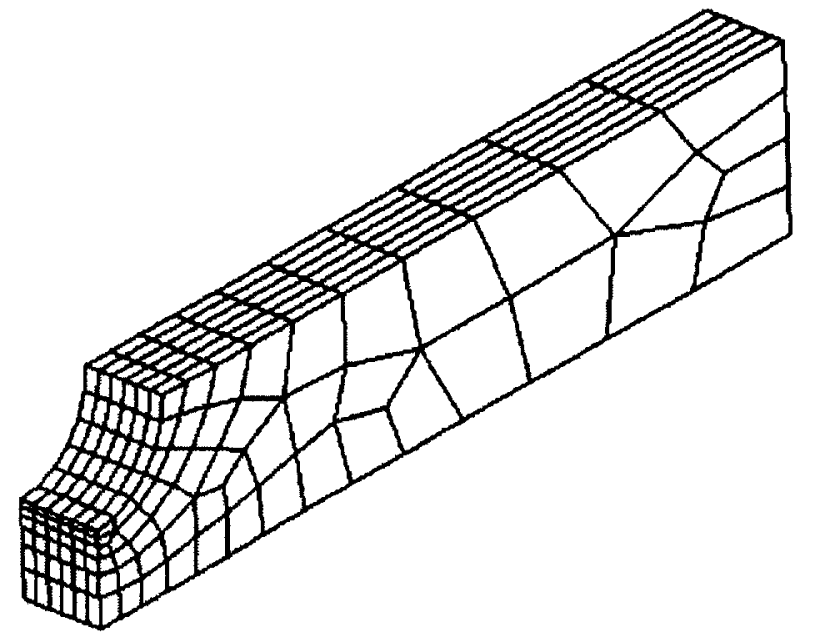

d) FEM for 3D problem, 392 elements, 1925 nodes

Figure 2.1: Typical BEM and FEM meshes for 2D and 3D problems 

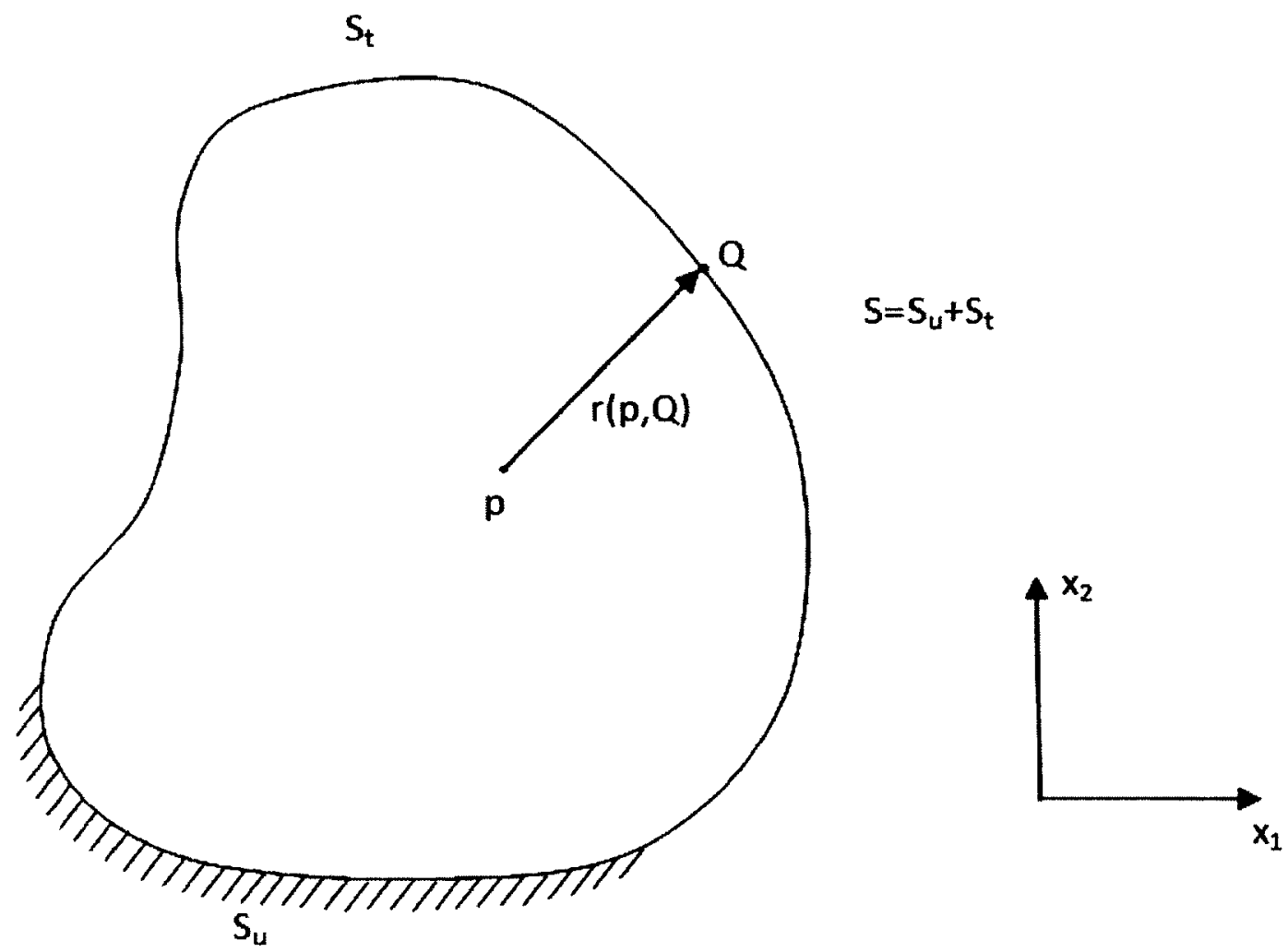

Figure 2.2: Boundary conditions imposed on surface of domain 


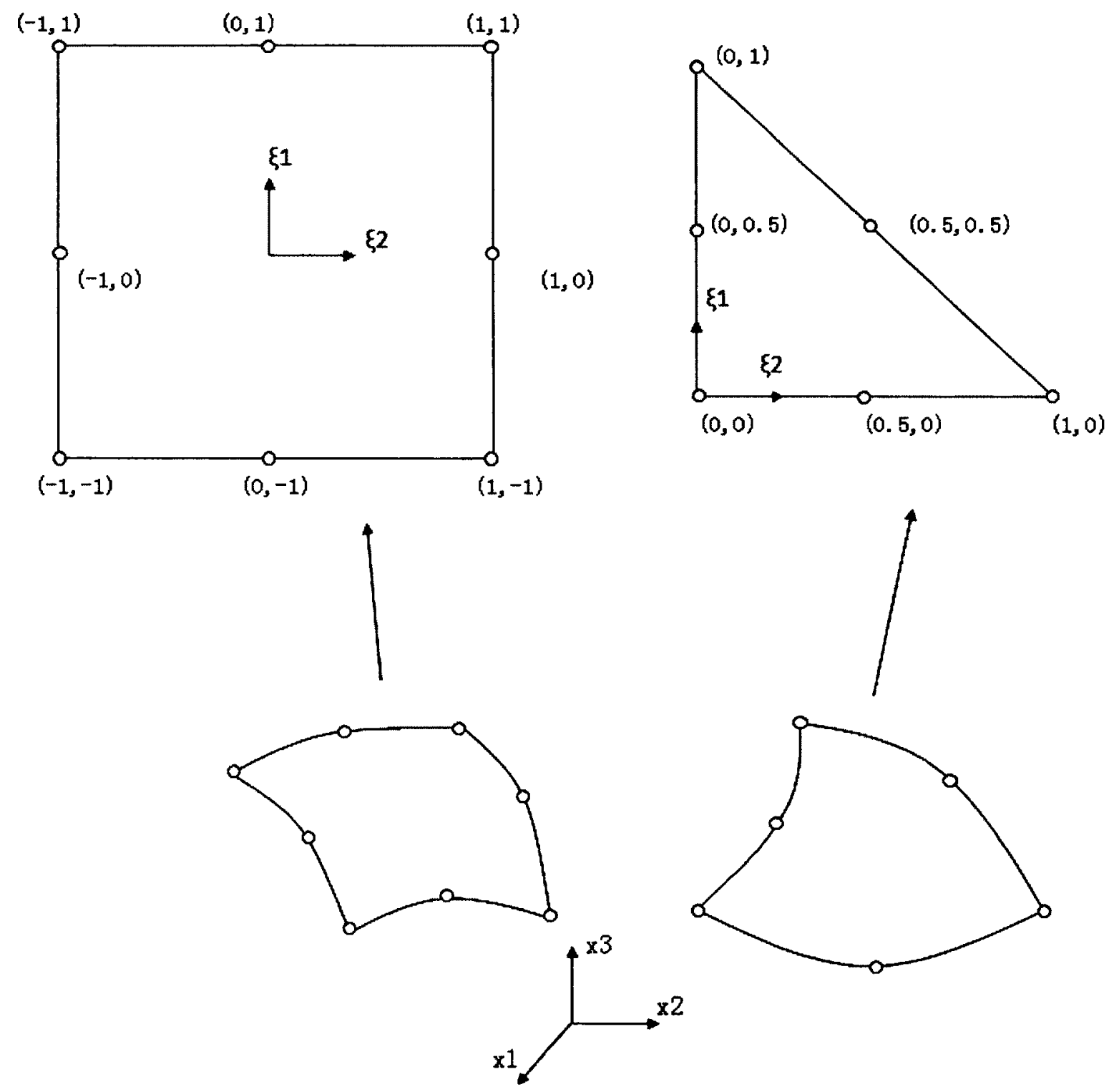

Figure 2.3: Local coordinate system of quadrilateral and triangular elements 


\section{Chapter 3}

\section{Self-regularization for Interior Points}

\subsection{Introduction}

In this chapter, the self-regularization scheme for interior point solutions proposed by Cruse and Richardson (1996) in two dimensions is extended to three dimensions. Using this method, when an interior point is close to the boundary, the near-singular kernels are regularized, therefore standard integration scheme can be used without any mesh refinement or special analytical transformation. The details of the regularization process are described in this chapter. Several numerical examples are presented to show the accuracy obtained by this method.

\subsection{Self-regularization of Interior Points in Three Dimensions}

The Somigliana identities provides the displacements and stresses at an interior point $p$ in terms of surface tractions and displacements. They are presented here again, as follows:

$$
u_{j}(p)=-\int_{S} u_{i}(Q) T_{j i}(p, Q) d S(Q)+\int_{S} t_{i}(Q) U_{j i}(p, Q) d S(Q)
$$


and

$$
\sigma_{i j}(p)=-\int_{S} u_{k}(Q) S_{k i j}(p, Q) d S(Q)+\int_{S} t_{k}(Q) D_{k i j}(p, Q) d S(Q)
$$

where the kernel functions $T_{i j}, U_{i j}, D_{k i j}$ and $S_{k i j}$ are given in Chapter 2. When an interior point is close to the boundary, results from the numerical evaluation of the integrals will deteriorate due to the rapid variation of these singular kernels. The selfregularized displacement identity for two dimensions subtracts and adds back terms of a rigid body motion as follows (Cruse and Richardson 1996):

$$
u_{j}(p)-u_{j}(P)=-\int_{S}\left[u_{i}(Q)-u_{i}(P)\right] T_{j i}(p, Q) d S(Q)+\int_{S} t_{i}(Q) U_{j i}(p, Q) d S(Q)
$$

where $p$ is the interior point, $P$ the regularization point at the boundary, and $Q$ the field point. Similarly, a regularized stress identity is proposed in Richardson and Cruse (1999). This regularized form is obtained by subtracting and adding back simple solutions corresponding to a constant stress state where the stress field is constant throughout the elastic body and the displacements are linearly distributed. The regularized stress identity is expressed as

$\sigma_{i j}(p)-\sigma_{i j}(P)=-\int_{S}\left[u_{k}(Q)-u_{k}^{L}(Q)\right] S_{k i j}(p, Q) d S(Q)+\int_{S}\left[t_{k}(Q)-t_{k}^{L}(Q)\right] D_{k i j}(p, Q) d S(Q)$

where $u_{k}^{L}(Q)$ and $t_{k}^{L}(Q)$ are displacement and traction solutions to the constant stress state, respectively. They are expressed as

$$
u_{k}^{L}(Q)=u_{k}(P)+u_{k, m}^{L}(P)\left[x_{m}(Q)-x_{m}(P)\right]
$$

and

$$
t_{k}^{L}(Q)=\sigma_{k m}(P) n_{m}(Q)
$$


where $n_{m}(Q)$ is the unit outward normal at integration point $Q$ and $u_{k, m}(P)$ are displacement gradients at the regularization point $P$, which will be obtained from the boundary values later.

The self-regularized displacement and stress identities has been tested successfully on simple two-dimensional elasticity problems by Richardson and Cruse (1999). It should be noted, however, that the singularity of all the kernel functions are one order higher in three dimensions than the corresponding ones in two dimensions. In Equation (3.3), $U_{i j}(P, Q)$ on the right-hand side is not regularized, since it is only of the order of $\ln (1 / r)$ in two dimensions. In three dimensions, however, this kernel is one order higher and becomes of order $r^{-1}$. It is useful for this kernel to be regularized as well, even though the associated integral is only weakly singular. Using the same form of adding and subtracting constant stress state terms to the stress identity, the following expression is obtained:

$u_{j}(p)-u_{j}^{L}(p)=-\int_{S}\left[u_{i}(Q)-u_{i}^{L}(Q)\right] T_{j i}(p, Q) d S(Q)+\int_{S}\left[t_{i}(Q)-t_{i}^{L}(Q)\right] U_{j i}(p, Q) d S(Q)$

where $u_{i}^{L}(Q)$ and $t_{i}^{L}(Q)$ are given before, and $u_{j}^{L}(p)$ is defined similarly as

$$
u_{j}^{L}(p)=u_{j}(P)+u_{j, m}(P)\left[x_{m}(p)-x_{m}(P)\right]
$$

where $u_{j, m}(P)$ is again the displacement derivatives at point $P$. Now all the kernels are completely regularized and the interior points can be taken close to the boundary without any concerns of the singularities.

The process for two-dimensional problems can be seen in Richardson and Cruse (1999), Yu (2006), and Shah (2007). Here the process is extended to three dimensions 
as it is slightly different than that in two dimensions.

At point $P$, tractions and displacements are interpolated by the nodal values of the element containing $P$ using the shape functions, i.e.:

$$
t_{i}=t_{i}^{c} N^{c}\left(\xi_{1}, \xi_{2}\right) \quad c=1,8 \text { or } 6
$$

The relation between tractions and displacement gradients is

$$
t_{i}=\sigma_{i j} n_{j}=\left[\frac{2 \mu \nu}{1-2 \nu} u_{k, k} \delta_{i j}+\mu\left(u_{i, j}+u_{j, i}\right)\right] n_{j}
$$

The relation between the displacement gradients in local and elemental coordinate systems is

$$
\frac{\partial u_{i}\left(\xi_{1}, \xi_{2}\right)}{\partial \xi_{j}}=\sum_{c=1}^{8 \text { or } 6} \frac{\partial N_{c}\left(\xi_{1}, \xi_{2}\right)}{\partial \xi_{j}}\left(u_{i}\right)_{c}=\frac{\partial u_{i}}{\partial x_{k}} \frac{\partial x_{k}}{\partial \xi_{j}}
$$

Combining the above two equations, a set of nine equations to obtain $u_{k, m}$ can be expressed in matrix form as follows: 


$$
\left\{\begin{array}{c}
t_{1} \\
t_{2} \\
t_{3} \\
\frac{\partial u_{1}}{\partial \xi_{1}} \\
\frac{\partial u_{2}}{\partial \xi_{1}} \\
\frac{\partial u_{3}}{\partial \xi_{1}} \\
\frac{\partial u_{1}}{\partial \xi_{2}} \\
\frac{\partial u_{2}}{\partial \xi_{2}} \\
\frac{\partial u_{3}}{\partial \xi_{2}}
\end{array}\right\}=\left[\begin{array}{ccccccccc}
\frac{2 \mu(1-\nu)}{1-2 \nu} n_{1} & \mu n_{2} & \mu n_{3} & \mu n_{2} & \frac{2 \mu \nu}{1-2 \nu} n_{1} & 0 & \mu n_{3} & 0 & \frac{2 \mu \nu}{1-2 \nu} n_{1} \\
\frac{2 \mu \nu}{1-2 \nu} n_{2} & \mu n_{1} & 0 & \mu n_{1} & \frac{2 \mu(1-\nu)}{1-2 \nu} n_{2} & \mu n_{3} & 0 & \mu n_{3} & \frac{2 \mu \nu}{1-2 \nu} n_{2} \\
\frac{2 \mu \nu}{1-2 \nu} n_{3} & 0 & \mu n_{1} & 0 & \frac{2 \mu \nu}{1-2 \nu} n_{2} & \mu n_{2} & \mu n_{1} & \mu n_{2} & \frac{2 \mu(1-\nu)}{1-2 \nu} n_{3} \\
\frac{\partial x_{1}}{\partial \xi_{1}} & 0 & 0 & \frac{\partial x_{2}}{\partial \xi_{1}} & 0 & 0 & \frac{\partial x_{3}}{\partial \xi_{1}} & 0 & 0 \\
0 & \frac{\partial x_{1}}{\partial \xi_{1}} & 0 & 0 & \frac{\partial x_{2}}{\partial \xi_{1}} & 0 & 0 & \frac{\partial x_{3}}{\partial \xi_{1}} & 0 \\
0 & 0 & \frac{\partial x_{1}}{\partial \xi_{1}} & 0 & 0 & \frac{\partial x_{2}}{\partial \xi_{1}} & 0 & 0 & \frac{\partial x_{3}}{\partial \xi_{1}} \\
\frac{\partial x_{1}}{\partial \xi_{2}} & 0 & 0 & \frac{\partial x_{2}}{\partial \xi_{2}} & 0 & 0 & \frac{\partial x_{3}}{\partial \xi_{2}} & 0 & 0 \\
0 & \frac{\partial x_{1}}{\partial \xi_{2}} & 0 & 0 & \frac{\partial x_{2}}{\partial \xi_{2}} & 0 & 0 & \frac{\partial x_{3}}{\partial \xi_{2}} & 0 \\
0 & 0 & \frac{\partial x_{1}}{\partial \xi_{2}} & 0 & 0 & \frac{\partial x_{2}}{\partial \xi_{2}} & 0 & 0 & \frac{\partial x_{3}}{\partial \xi_{2}}
\end{array}\right]\left\{\begin{array}{c}
u_{1,1} \\
u_{2,1} \\
u_{3,1} \\
u_{1,2} \\
u_{2,2} \\
u_{3,2} \\
u_{1,3} \\
u_{2,3} \\
u_{3,3}
\end{array}\right\}
$$

Inversion of the $(9 \times 9)$ matrix $\left[\mathbf{A}^{*}\right]$ gives values of $u_{k, m}$ in terms of nodal displacements and tractions of the element containing the regularization point. They are then used in Equation (3.5) to get $u_{l}^{k}$. The $t_{l}^{k}$ terms are computed using Equations (3.10) with $n_{j}$ being the unit outward normal at point $Q$ as in Equation (3.6).

In two-dimensional analysis, the regularization point is simply chosen as the node closest to the interior point ( $\mathrm{Yu}$ 2006, Shah 2007). In three dimensions, however, it was found out that the nearest node cannot always be chosen as the regularization point due to the fact that the kernels are one order higher than those in two dimensions, making their variations more rapid. This is especially true when the interior point is very close to the boundary. This can be further explained here. Figure 3.1 shows a two-dimensional element on which regularization point $P$ is the closest to the interior point $p$. If one takes the example of $T_{i j}$ which is of 
order of $r^{-2}(p, Q)$, the singularity is regularized by the term $\left[u_{i}(Q)-u_{i}(P)\right]$ and the Jacobian $J\left(\xi_{1}, \xi_{2}\right)$. As the interior point gets close to the boundary, $r(p, Q)$ will tend to equal to $r(P, Q)$, which cancels the singularity precisely. However, if $P$ is located somewhere else, for example a boundary node $P^{\prime}$ in Figure 3.1, $r\left(P^{\prime}, Q\right)$ may not be small enough to minimize the large quantity produced by $1 / r^{2}$ in the $T_{i j}$ terms. Therefore the closest point has to be found so that the $r(p, Q)$ is the smallest distance from $p$ to the boundary. In other words, the normalized vector $\vec{a}$ from $p$ to $P$ should be parallel to the outward normal at $P$. This is done by making components in each direction of these two vectors equal, i.e.

$$
a_{i}=n_{i}(P)
$$

An algorithm has been developed to find such a point, which first identifies the closest element to the interior point, then divides the element into 10 by 10 grids, search for the closest intersection point $P_{1}$ on the grid, then divides a smaller area around that point into a smaller grid in which a closer point $P_{2}$ is searched for. This process is repeated until the difference between $a_{i}$ and $n_{i}(P)$ falls under a certain threshold, so that these two vectors are sufficiently parallel. In this study, this threshold was set to be 0.00001 . This process is illustrated in Figure 3.2. Using this algorithm, the regularization point is mostly located inside an element where the displacement derivatives and stresses are continuous, so that the smoothness requirement of the regularization point is met, as discussed in Cruse and Richardson (1996). 


\subsection{Numerical Examples}

The self-regularization scheme described above has been implemented into the BEM code based on displacement-BIE as used in Tan and Fenner (1978) for general threedimensional isotropic elastostatics. $4 \times 4$ Gaussian and 13-point Hammer-Stroud quadrature are employed for quadrilateral and triangular elements, respectively. To verify this technique, several numerical problems involving different geometries and boundary conditions are tested. For all the cases, Young's modulus is set to 1000 units and Poisson's ratio is 0.3 unless specified otherwise. In each case, interior points are located so that the distance to the surface is gradually decreased. For comparison, the results without using self-regularization are also presented for some cases so that one can observe the deterioration of the solutions without the regularization of the singular kernels. The results are also compared with the exact solutions that can be found in literature to demonstrate the accuracy obtained with this method.

\section{(A) A Cube under Uniform Stress}

A simple solid cube with a dimension of 1 unit in each direction under uniform applied stress on its sides is tested first. The block is meshed by only 6 elements and 20 nodes, as shown in Figure 3.3. Two cases, one with uniform tensile stresses $\sigma_{11}=1, \sigma_{22}=2, \sigma_{33}=3$ and one with shear stresses $\sigma_{12}=\sigma_{13}=\sigma_{23}=1$ are tested. In the first case, three surfaces are constrained in three directions and the other three corresponding parallel surfaces are loaded with tensile stresses. In the shear loading case, the appropriate nodal constraints are applied.

Several interior points are located at positions ranging from the center of the block to close to the surfaces, as shown in Figure 3.3. The stresses are uniform within the domain, and the displacements are obtained from Hooke's law and 
strain-displacement relations as:

$$
u_{i}=\epsilon_{i j} d_{j}=\frac{1}{E}\left[\sigma_{i j}-\nu\left(\sigma_{k k} \delta_{i j}-\sigma_{i j}\right)\right] d_{j}
$$

where $d_{j}$ is the distance from a fixed point, set at the origin. The calculated stresses and displacements at the interior points are presented in Tables 3.1 through 3.4 for the two cases, both with and without self-regularization, and are compared with exact solutions.

\section{(B) Cantilever Beam under a Shear Load}

The second problem is a cantilever beam with one end fully constrained and the other subject to an unit shear load. Poisson's ratio is set to zero to enable comparison with the results of one-dimensional simple beam theory. The beam has a length of 8 units and its height and width are both 1 unit. The interior points are located at a crosssection whose distance from the fixed end is 3 , and are positioned from the lower surface to the upper surface, as shown in Figure 3.4 (a). Figure 3.4 (b) shows the mesh of the beam with 72 elements and 218 nodes. According to the beam-bending theory, the axial stress $\sigma_{x}$ and lateral displacement $v$ at a point with distance $x$ from the fixed end and distance $y$ from the neutral axis are:

$$
\sigma_{x}=\frac{M y}{I}=\frac{F(l-x) y}{I}
$$

and

$$
v=\frac{F x^{2}(3 l-x)}{6 E I}
$$

respectively, where $F$ is the shear load applied at the free end, $l$ the total length of the beam, and $I$ the moment of inertia of the cross-section. 
Table 3.5 presents the axial stress and vertical displacement at these interior points obtained, with and without self-regularization. The exact solutions calculated from the above two equations are also listed for comparison.

\section{(C) Internally Pressurized Cylinder and Sphere}

Two pressurized vessels are tested here in the shapes of a cylinder and a sphere. The case considered are for outer to inner radius ratio, $K=r_{o} / r_{i}$, of 2 and 1.5 for the cylinder and the sphere, respectively, where the inner radius $r_{i}$ is arbitrarily taken to be 1 , and $r_{o}$ is the outer radius. Due to symmetry, only $1 / 4$ of the cylinder and $1 / 8$ of the sphere are modeled. Meshes for the problems are shown in Figure 3.5 (b) and (c). The cylinder is meshed with 32 elements and 92 nodes; the mesh for the sphere has 48 elements and 146 nodes. For both cases, interior points are located on a radial line from the inner radius to the outer radius, as shown in Figure 3.5 (a). The exact solutions for hoop stress distribution in a pressurized cylinder can be expressed as:

$$
\sigma_{\theta}=\frac{p_{i}-p_{o} K^{2}}{K^{2}-1}-\frac{K^{2}\left(p_{o}-p_{i}\right)}{K^{2}-1}\left(\frac{r_{i}}{r}\right)^{2}
$$

and radial stress as

$$
\sigma_{r}=\frac{p_{i}-p_{o} K^{2}}{K^{2}-1}+\frac{K^{2}\left(p_{o}-p_{i}\right)}{K^{2}-1}\left(\frac{r_{i}}{r}\right)^{2}
$$

where $p_{i}$ and $p_{o}$ are internal and external pressure, respectively, and $r$ is the radial distance. In this example, the upper and lower end surfaces of the cylinder are constrained in the axial direction, so that the axial stress $\sigma_{a}$ is obtained for planestrain state from:

$$
\sigma_{a}=\nu\left(\sigma_{\theta}+\sigma_{r}\right)
$$


For a pressurized sphere, the hoop stress and radial stress are given by:

$$
\sigma_{\theta}=\frac{-p_{o} K^{3}+p_{i}}{K^{3}-1}-\frac{\left(p_{o}-p_{i}\right) K^{3}}{2\left(K^{3}-1\right)}\left(\frac{r_{i}}{r}\right)^{3}
$$

and

$$
\sigma_{r}=\frac{-p_{o} K^{3}+p_{i}}{K^{3}-1}+\frac{\left(p_{o}-p_{i}\right) K^{3}}{K^{3}-1}\left(\frac{r_{i}}{r}\right)^{3}
$$

The radial displacement $u_{r}$ can be obtained from hoop strain, i.e.

$$
\epsilon_{\theta}=\frac{u_{r}}{r}
$$

where

$$
\epsilon_{\theta}=\frac{1}{E}\left[\sigma_{\theta}-\nu\left(\sigma_{r}+\sigma_{\theta}\right)\right]
$$

or in case of the cylinder,

$$
\epsilon_{\theta}=\frac{1}{E}\left[\sigma_{\theta}-\nu\left(\sigma_{r}+\sigma_{a}\right)\right]
$$

The displacements and stresses at the interior points obtained from self-regularization for the cases are presented in Tables 3.6 and 3.7, respectively, and are compared with the exact solutions obtained from the above equations.

\section{(D) Stress Concentration Problems}

Stress concentrations are commonly seen in many engineering situations involving rapid changing geometries such as notches, grooves or holes. Two examples are given here to demonstrate the accuracy and efficiency of the self-regularization technique for interior point solution. In both cases, the boundary solutions from BEM and the interior point solutions are compared to a finite element analysis using ANSYS on the same numerical solution domain. 
The first problem being treated is a double-notched bar under tension, as shown in Figure 3.6. The notch radius-to-height ratio, $r / H$, is set to 0.5 where $r=1$. The thickness $t$ is 2. Due to symmetry, only one quarter of the model is meshed, as indicated by the shaded area in Figure 3.6, although the numerical problem can be further reduced by taking advantage of symmetry about the other planes. The partial model being treated is shown in Figure 3.7, whose modeled length is 10 units. The boundary element mesh shown in Figure 3.8 has 172 elements and 518 nodes. The corresponding finite element mesh, as shown in Figure 3.9, has 552 SOLID186 20-node brick quadratic elements and 3035 nodes.

Two groups of interior points are investigated. The first group is located close to the line of stress concentration along the thickness of the bar, through which the stress distribution is plotted in Figure 3.10. Also shown in the figure are the stress variations extracted at the boundary nodes along the thickness of both BEM and FEM meshes. The second group of interior points lie at the middle plane of the bar $(z=0)$ and are located from the line of stress concentration $(y=1)$ to the plane of symmetry $(y=2)$, whose stresses are calculated using the self-regularization technique. The "path operation" function in ANSYS is used to map the first principal stress onto these interior points. The stress variations from BEM interior point solution and FEM path results are plotted in Figure 3.11. The maximum stress occurring at the middle plane is 3.403 from BEM boundary solution and 3.386 from interior point solution, while in FEM it is 3.452. As seen in Figure 3.11, the deviation of the first principal stresses between the BEM and FEM is less than $2 \%$ along the designated path.

The second stress concentration problem is a long cylindrical bar with a spherical cavity subject to tension at the ends, as shown in Figure 3.12. The diameter ratio 
$d / h$ is 0.4 , where $h=1$. The modeled length of the bar is 6 units. Only a quarter of the domain is modeled due to symmetry. The boundary element mesh is shown in Figure 3.13; it has 380 nodes and 126 elements. The finite element mesh is shown in Figure 3.14; it has 5516 nodes and 1092 SOLID186 brick elements.

The first principal stress at the point of stress concentration obtained from nodal values of BEM and FEM are shown in Table 3.8 , along with that at an interior point very close to the concentration point using the self-regularization technique. They are compared with the analytical solution presented in Pilkey (2008) here in Figure 3.15. The stress variation through a series of inteior points inside the body is plotted in Figure 3.16, whose radial position $r$ range from the cavity surface $(r=0.4)$ to the free surface $(r=1)$. Path operation is again used in ANSYS to recover stresses at these points. Figure 3.16 shows the stress variations from the BEM and FEM interior point solution. At the cavity surface, the maximum stresses obtained from the BEM and FEM are as in Table 3.8, with the former being more accurate.

\subsection{Discussion of Results}

As can be seen in the results of the numerical examples, the self-regularization technique successfully cancelled the singularities of the kernels. With this scheme, less than $2 \%$ errors in stresses and less than $0.2 \%$ errors in displacements are obtained, regardless of the distances from the boundary. The errors in the stresses are generally higher than that of the displacements due to the higher order singularities in the kernels. Note that in Table 3.6, the percentage errors for the radial stresses at points close to the external surface are not of practical significance because these stresses are almost close to zero. As comparison, the original BEM algorithm without self-regularization starts to fail very sharply as the distance from the boundary 
reaches to around $10 \%$ of the thickness of the local cross-section, especially for the stresses.

The stress concentration problems demonstrated that this regularization technique recovers very accurate results near or at the surface. The boundary element method is well-known to give more accurate results for the boundary solutions than the finite element method for the same level of surface discretization. Using a very coarse mesh, the self-regularization scheme with the boundary element method gives a stress distribution very close to that obtained from finite element analysis, which had employed significantly more elements and nodes. The interior point solution using the self-regularization provides even better results than the primary boundary solutions due to the fact that the interior of the domain remains continuous, and no approximation therein is assumed. This enables the investigation of stress distribution inside a body with very high accuracies without any modification to the surface meshes.

The algorithm for locating the regularization point in this study always searches for a surface point so that the vector from the interior point to this point is always perpendicular to the surface at $P$. This point is, in most cases, located within an element where stress continuity is satisfied. More importantly, it never locates the regularization point at a corner, where stress discontinuity is most severe. It was found out in this study that if an interior point is adjacent to an element boundary or even a boundary node, so that the regularization point has to be located there, among the elements sharing this boundary or the node, no effect on the final results has been found no matter which element the regularization point belongs to. This means that although displacement gradients are discontinuous across element boundaries, the stresses and displacements at interior points obtained using 
self-regularization are still very accurate. The variety of geometries and loading cases of the numerical examples above illustrated the successful application of the self-regularization scheme in BEM for the stress analysis of 3D elastostatics.

\subsection{Summary}

In this chapter, the self-regularization for the displacement-BIE proposed in Cruse and Richardson (1996) and Richardson and Cruse (1999) has been implemented in three-dimensional elastostatics, with some modifications to the simple solutions that are added and subtracted from the displacement identity. The detailed process of the procedure is described, including derivation of the displacement derivatives and consequently the constant stress state terms, as well as locating the regularization point on the boundary. Several numerical examples were presented to demonstrate the accuracy of this technique. They showed that the displacement and stress solutions for interior points remain very accurate even when they are very close to the surface. Unlike the original formulation without the self-regularization, neither mesh refinement nor special transformations are required to obtain such accurate results for these interior points.

The next chapter will discuss the traction boundary integral equation (TBIE) combined with self-regularization technique applied to three-dimensional problems. This can be seen as an extension of this regularization method. 


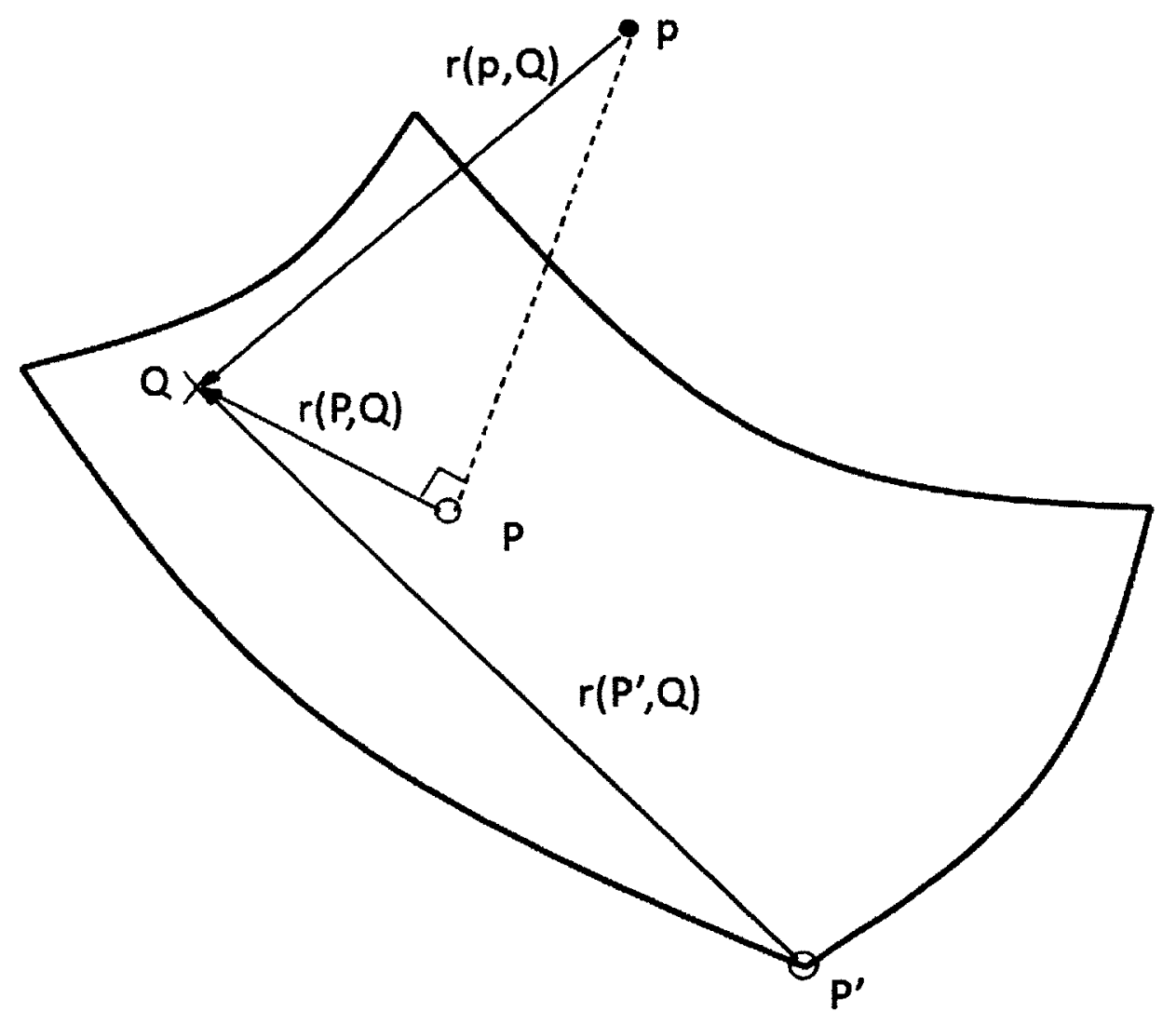

Figure 3.1: Schematic of regularization point 


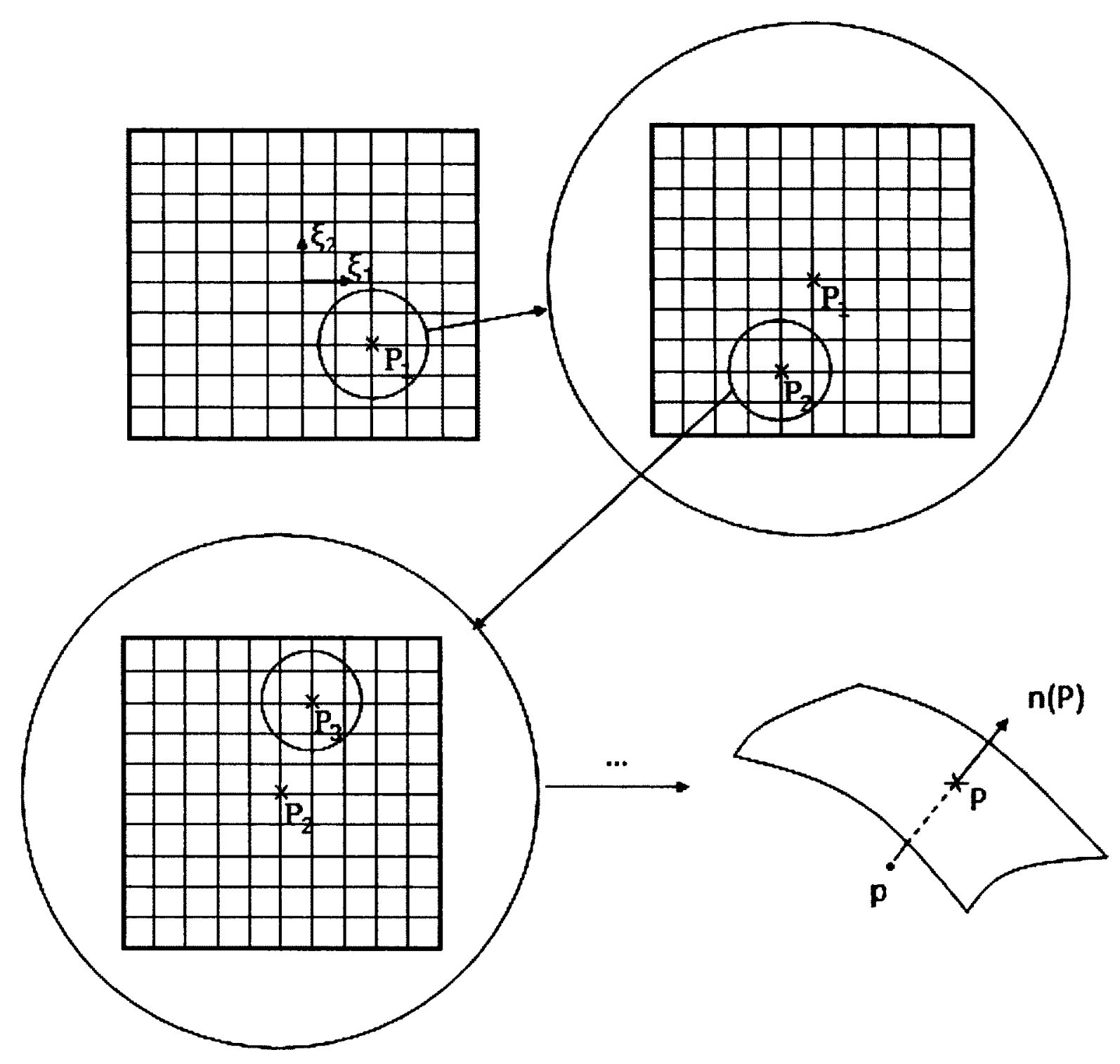

Figure 3.2: Locating of the regularization point 


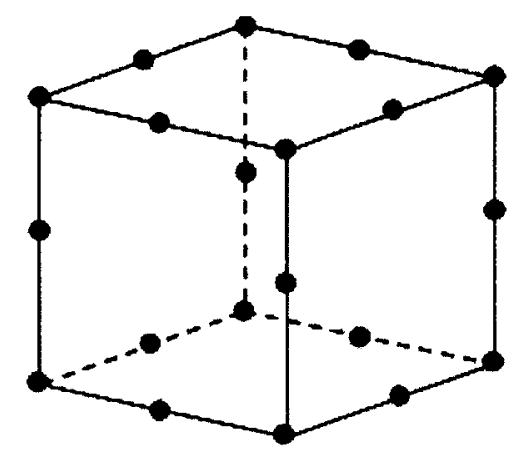

a) Mesh of a elastic cubic

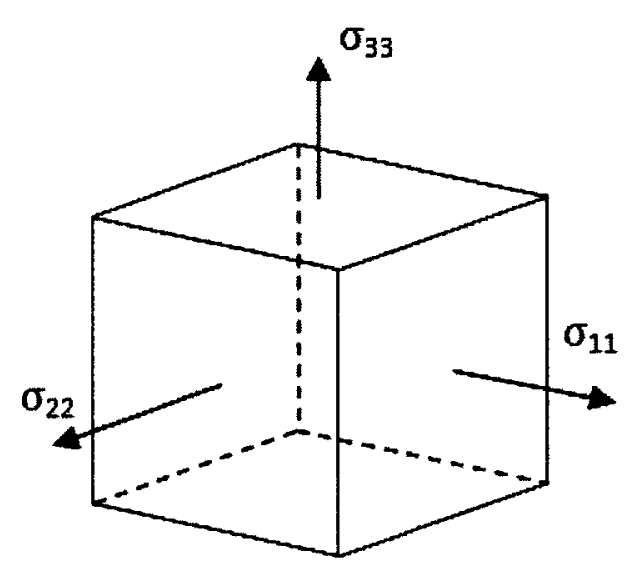

c) Applied uniform stress

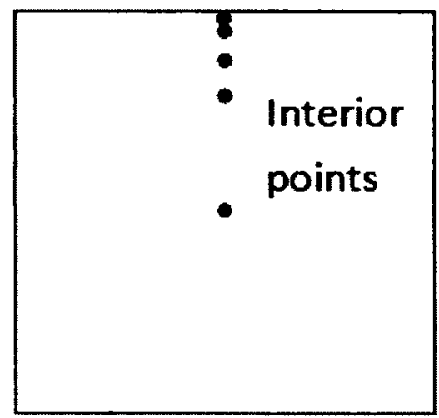

b) Interior points in a typical mid-plane

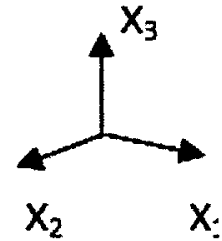

d) Applied uniform shear stresses

Figure 3.3: Schematic of the cubic body - Problem (A) 


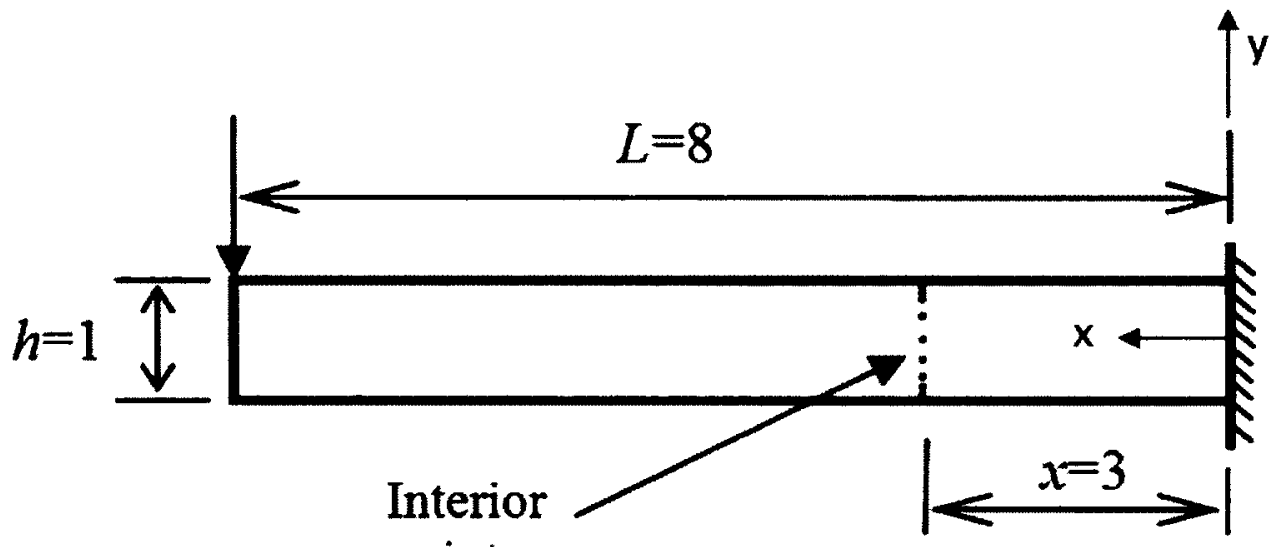
points

a) A cantilever beam-problem (B)

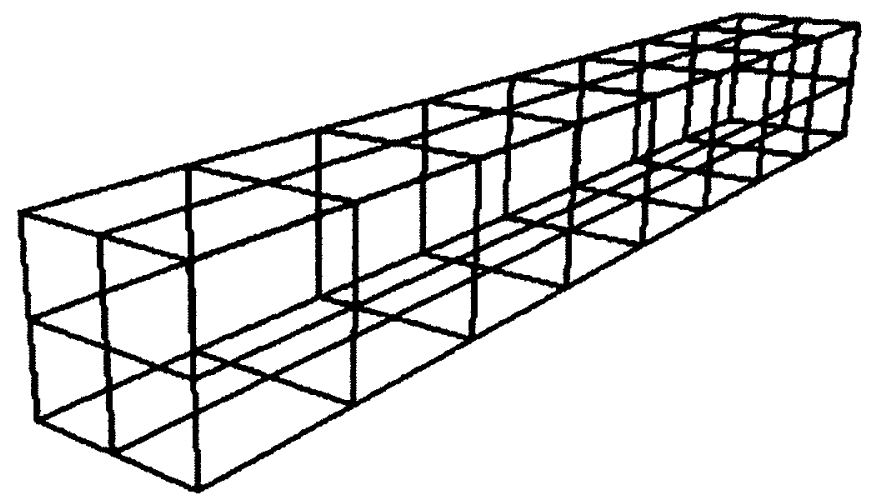

b) BEM mesh of the cantilever beam-problem (B)

Figure 3.4: Definition and mesh of the cantilever beam - Problem (B) 


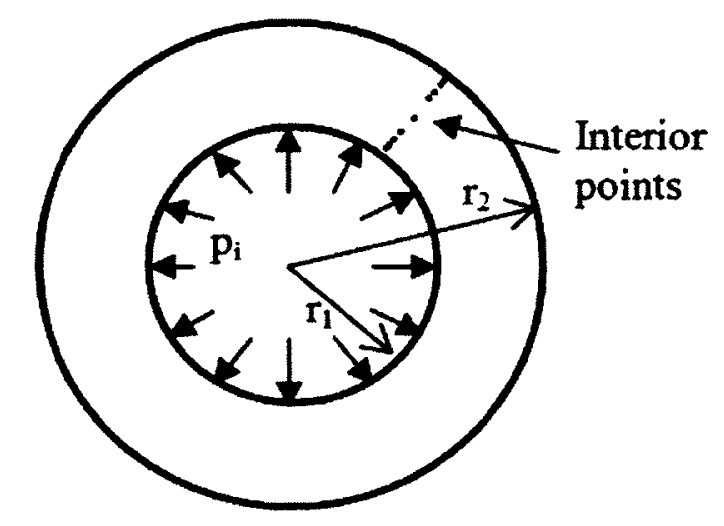

a) Schematic of the pressure vessels

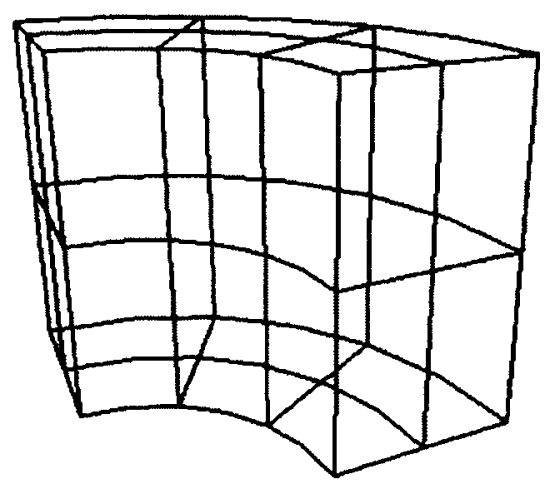

b) Mesh of the cylinder

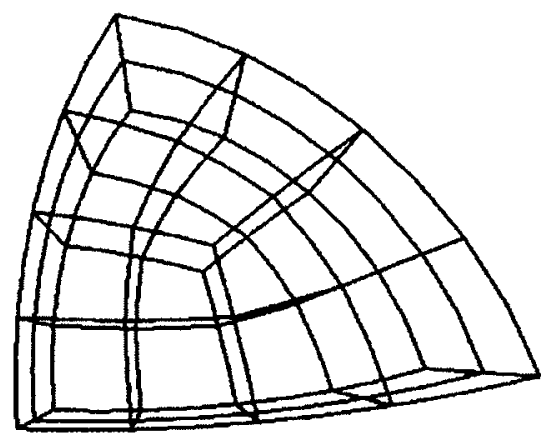

c) Mesh of the hollow sphere

Figure 3.5: Interior point locations and meshes of the pressurized vessels - Problem (C) 


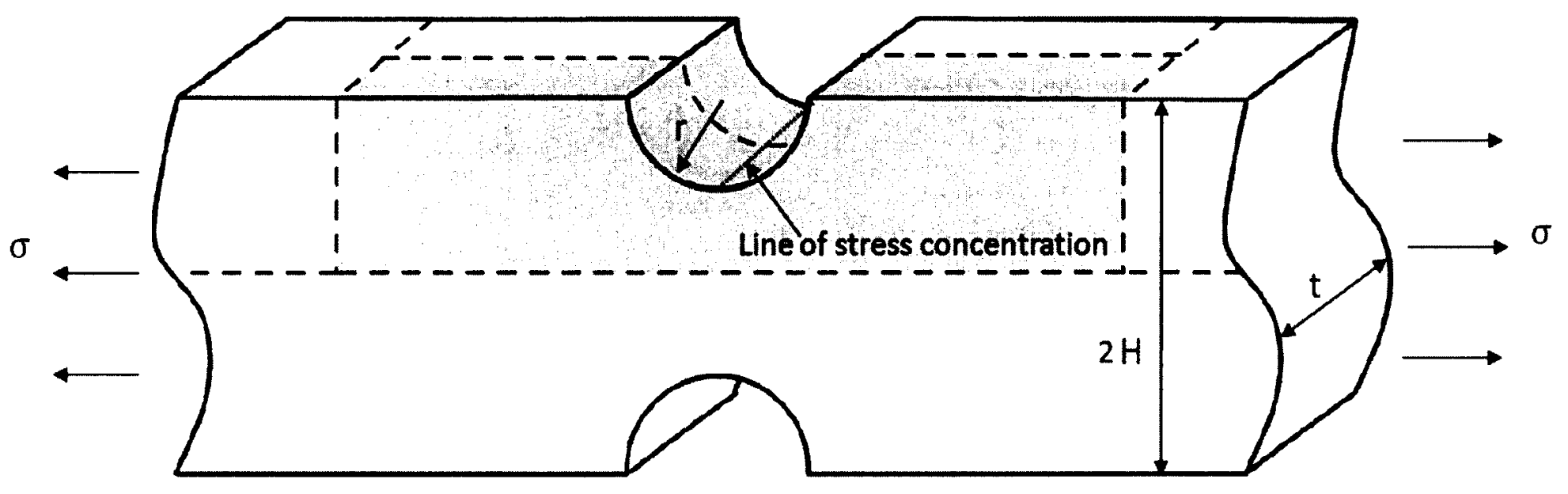

Figure 3.6: Schematic diagram of the double-notched bar - Problem (D-1) 


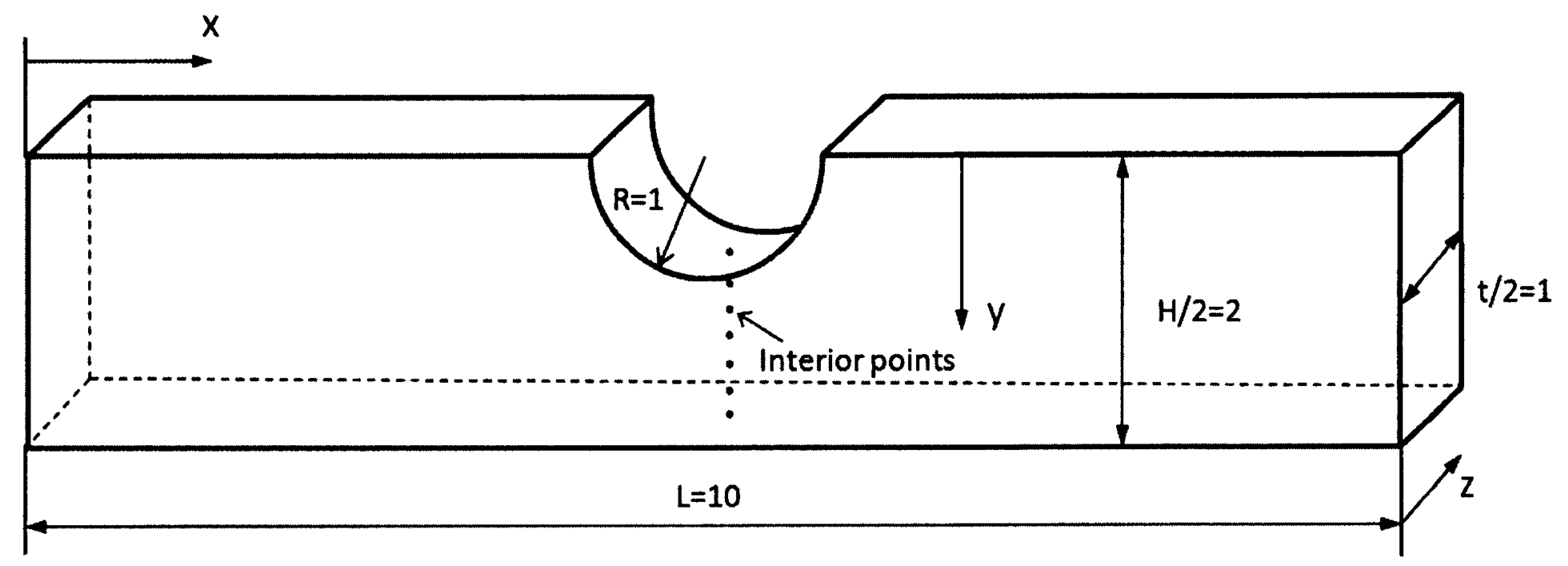

Figure 3.7: The modeled region of the double-notched bar - Problem (D-1) 


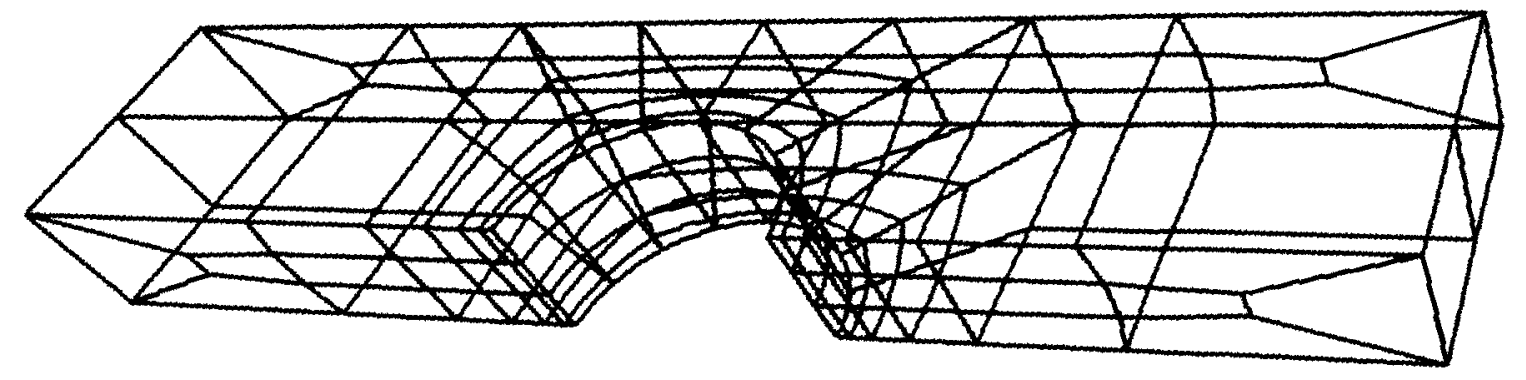

$$
\text { Figure 3.8: Boundary element mesh of the double-notched bar - Problem }(D-1)
$$




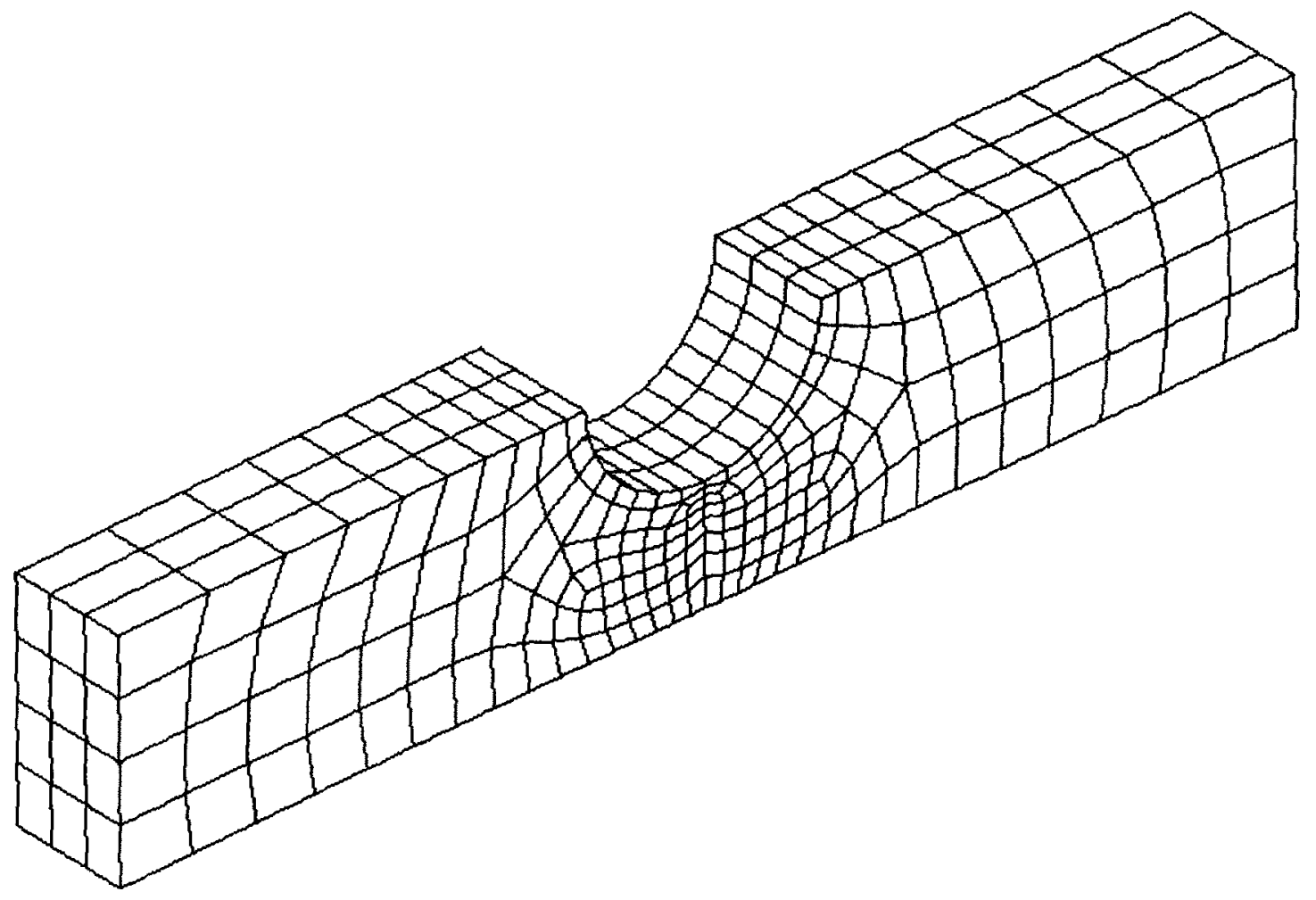

Figure 3.9: Finite element mesh of the double-notched bar - Problem (D-1) 


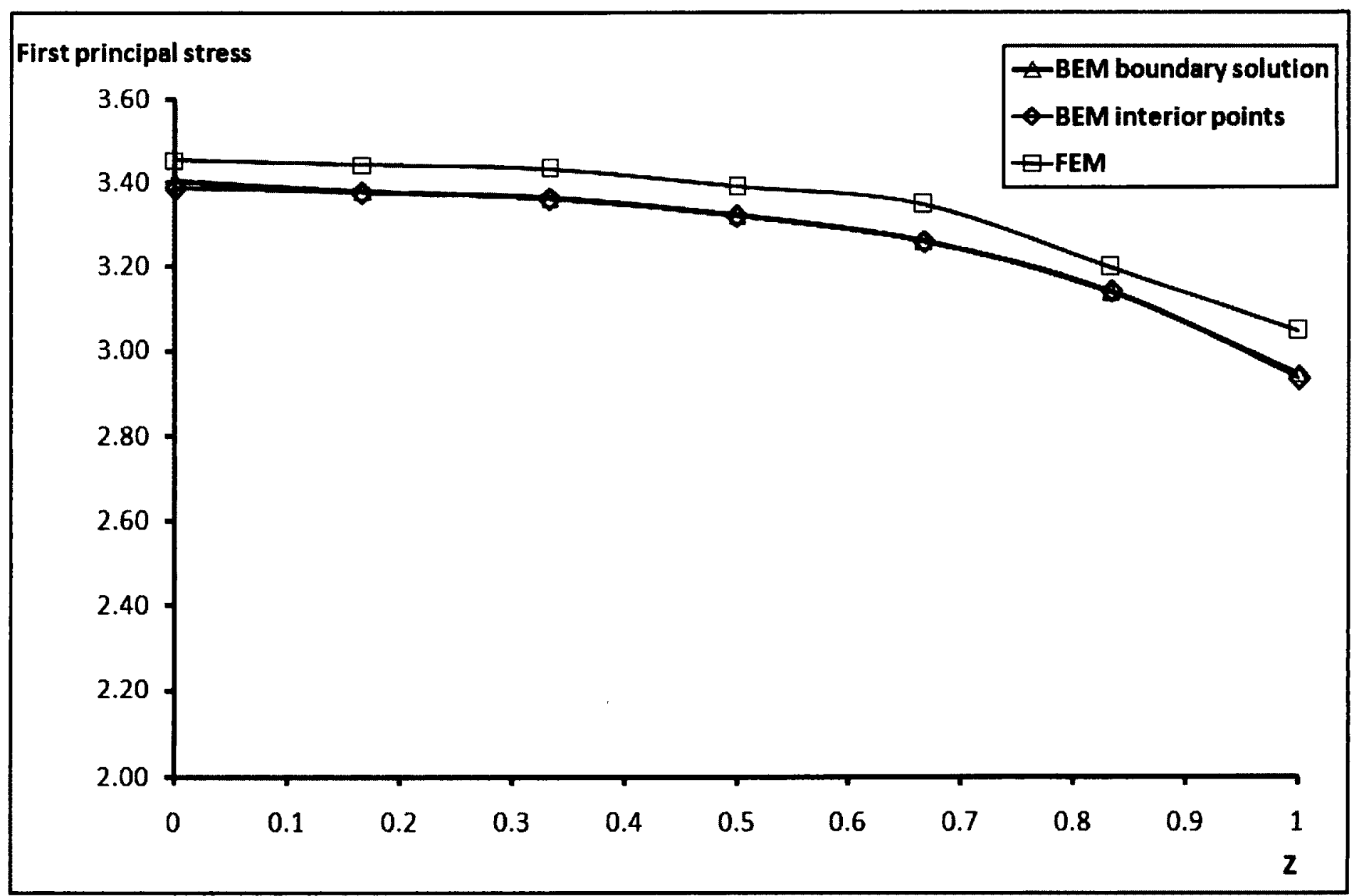

Figure 3.10: Stress variation along thickness of the double-notched bar - Problem (D-1) 


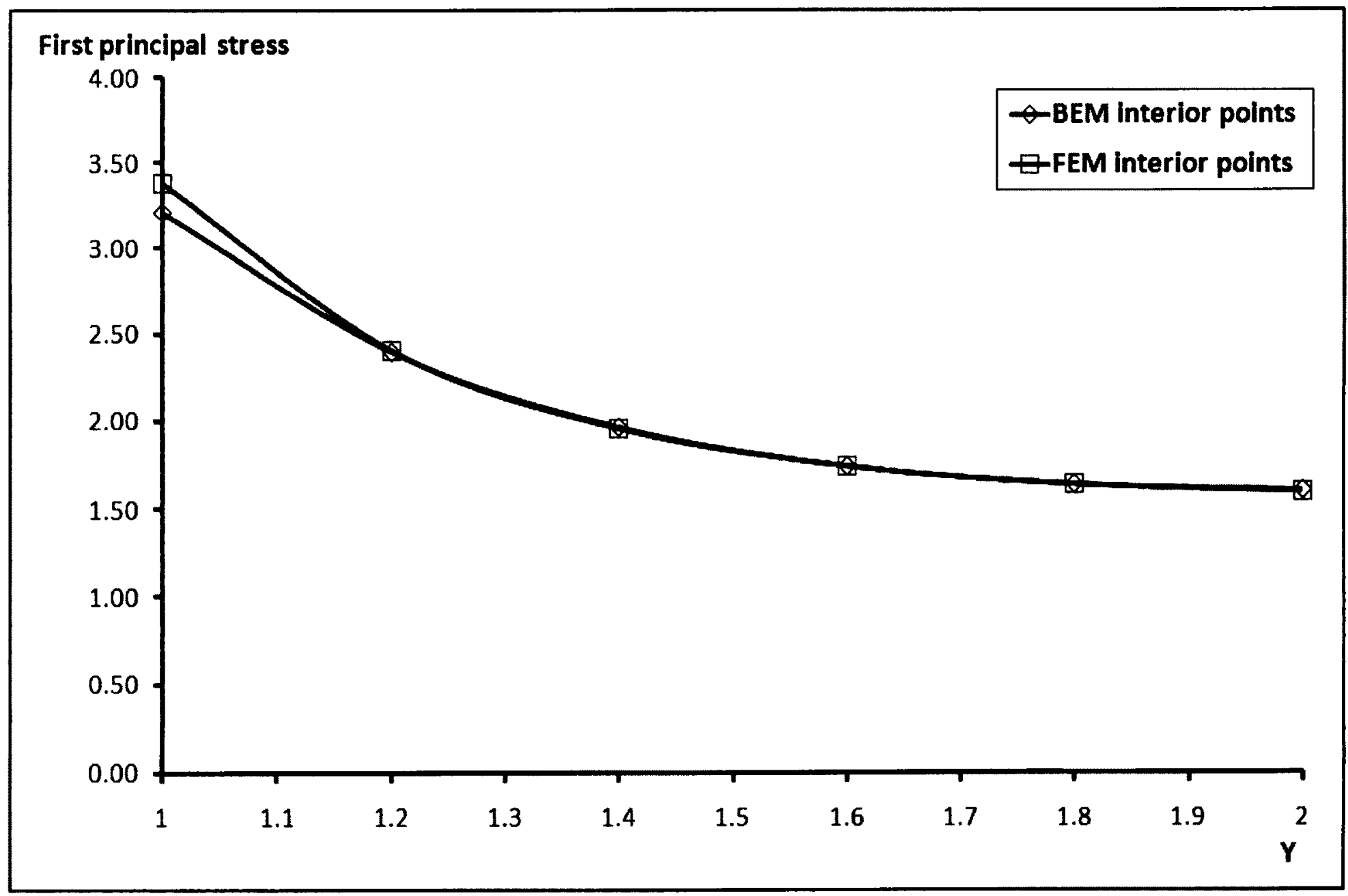

Figure 3.11: Stress variation at interior of the double-notched bar - Problem (D-1) 


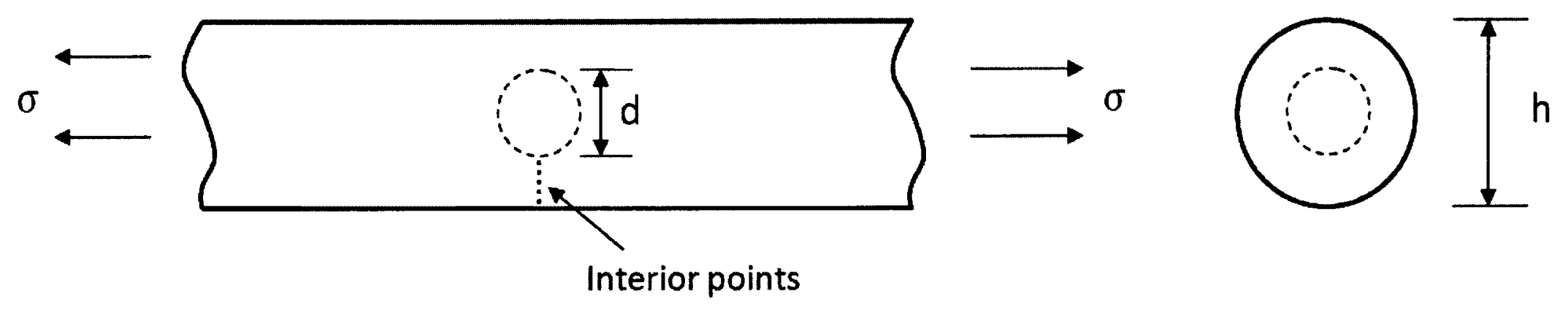

Figure 3.12: A cylinder with a spherical cavity - Problem (D-2) 


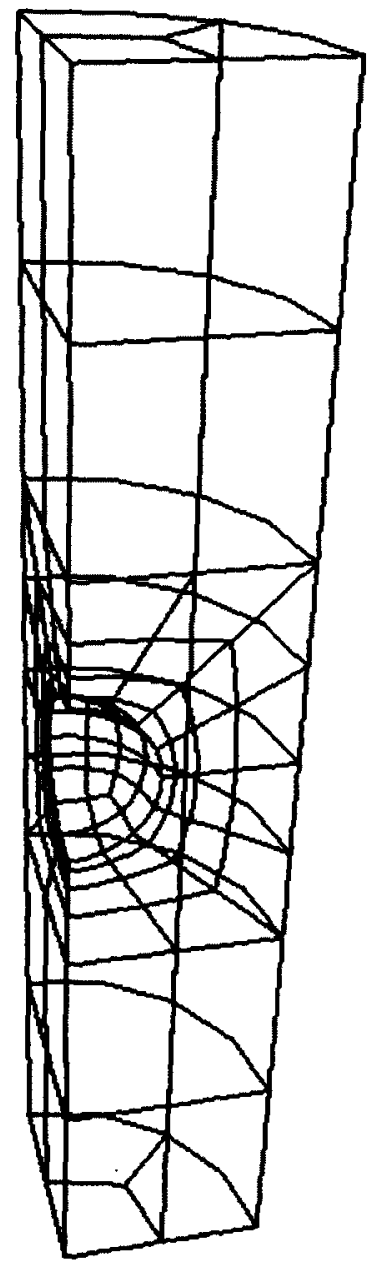

Figure 3.13: BEM mesh of the cylindrical bar with a spherical cavity - Problem (D-2) 


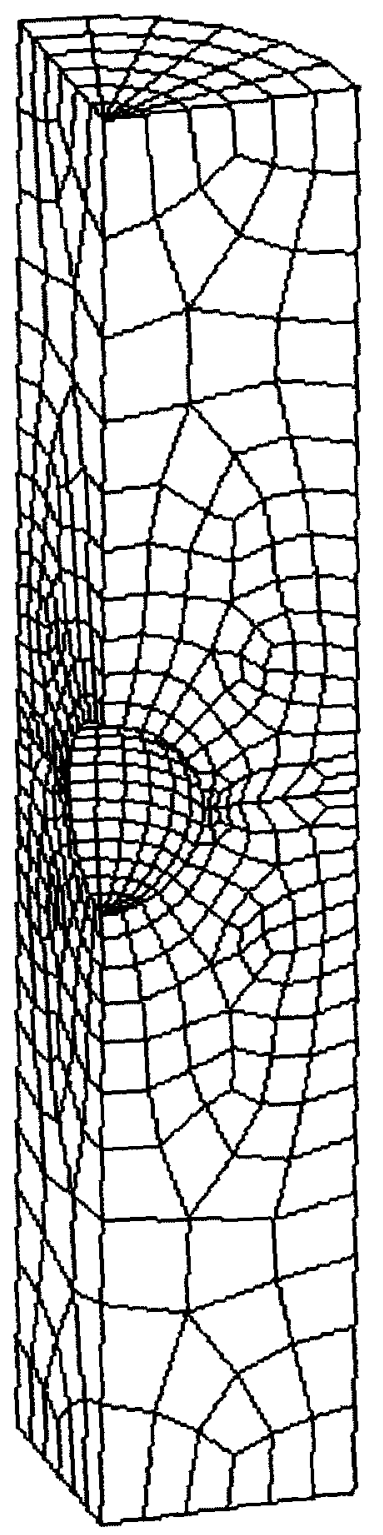

Figure 3.14: FEM mesh of the cylindrical bar with a spherical cavity - Problem (D-2) 


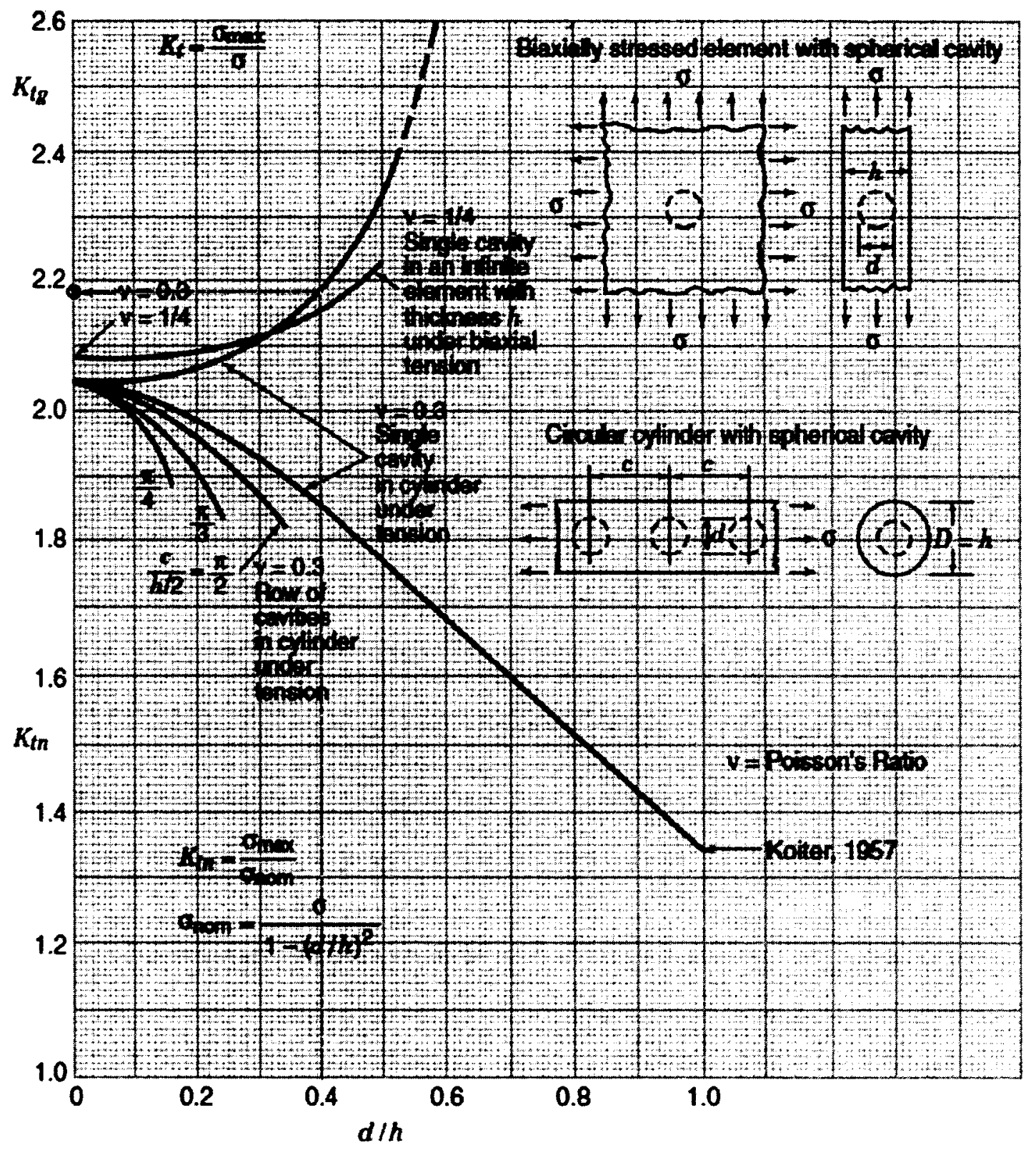

Figure 3.15: Analytical solution for the cylinder with spherical cavity (Pilkey 2008) 


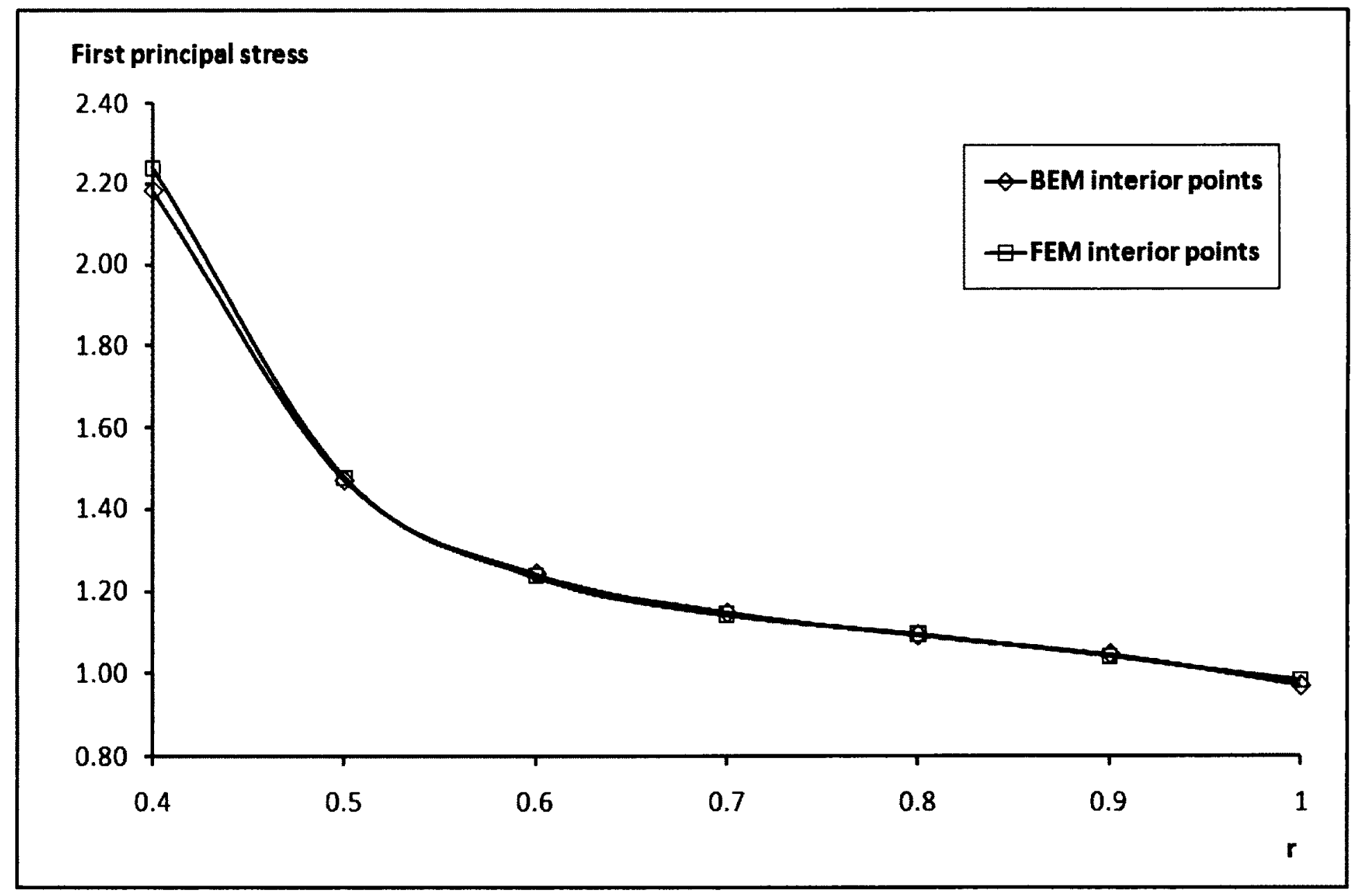

Figure 3.16: Stress distribution at the interior points of the cylinder with cavity by BEM and FEM - Problem (D-2) 


\begin{tabular}{|c|c|c|c|c|c|c|c|c|c|c|c|c|}
\hline & \multicolumn{9}{|c|}{ With self-regularization } & \multicolumn{5}{c|}{ Without self-regularization } \\
\hline Coordinates & $\sigma_{11}$ & $\sigma_{22}$ & $\sigma_{33}$ & $\sigma_{12}$ & $\sigma_{23}$ & $\sigma_{13}$ & $\sigma_{11}$ & $\sigma_{22}$ & $\sigma_{33}$ & $\sigma_{12}$ & $\sigma_{23}$ & $\sigma_{13}$ \\
\hline$(0.5,0.5,0.5)$ & 1.000 & 2.000 & 3.000 & $-8 \mathrm{E}-05$ & $6 \mathrm{E}-05$ & $-1 \mathrm{E}-05$ & 0.999 & 2.000 & 3.001 & $-8 \mathrm{E}-05$ & $6 \mathrm{E}-05$ & $-1 \mathrm{E}-05$ \\
$(0.9,0.5,0.5)$ & 1.001 & 2.000 & 3.000 & $1 \mathrm{E}-04$ & $9 \mathrm{E}-06$ & $7 \mathrm{E}-05$ & -5.376 & 3.302 & 4.398 & -1.16 & $8 \mathrm{E}-06$ & -3.05 \\
$(0.99,0.5,0.5)$ & 1.000 & 2.001 & 3.001 & $1 \mathrm{E}-04$ & $-9 \mathrm{E}-06$ & $-4 \mathrm{E}-05$ & $-7 \mathrm{E}+03$ & $1 \mathrm{E}+03$ & $1 \mathrm{E}+03$ & $-9 \mathrm{E}+02$ & $-3 \mathrm{E}-07$ & $-2 \mathrm{E}+03$ \\
$(0.999,0.5,0.5)$ & 1.000 & 2.001 & 3.001 & $1 \mathrm{E}-04$ & $-1 \mathrm{E}-05$ & $-4 \mathrm{E}-05$ & $-7 \mathrm{E}+06$ & $1 \mathrm{E}+06$ & $1 \mathrm{E}+06$ & $-9 \mathrm{E}+05$ & $-6 \mathrm{E}-07$ & $-2 \mathrm{E}+06$ \\
$(0.9999,0.5,0.5)$ & 1.000 & 2.001 & 3.001 & $1 \mathrm{E}-04$ & $-1 \mathrm{E}-05$ & $-4 \mathrm{E}-05$ & $-7 \mathrm{E}+09$ & $1 \mathrm{E}+09$ & $1 \mathrm{E}+09$ & $-9 \mathrm{E}+08$ & $-6 \mathrm{E}-07$ & $-2 \mathrm{E}+09$ \\
$(0.5,0.9,0.5)$ & 1.000 & 2.000 & 3.000 & $2 \mathrm{E}-05$ & $-2 \mathrm{E}-05$ & $-9 \mathrm{E}-06$ & -1.78 & 13.27 & 0.41 & $7 \mathrm{E}-01$ & -3.05 & $-8 \mathrm{E}-06$ \\
$(0.5,0.99,0.5)$ & 1.000 & 2.000 & 3.000 & $1 \mathrm{E}-04$ & $-1 \mathrm{E}-04$ & $-8 \mathrm{E}-06$ & $-2 \mathrm{E}+03$ & $1 \mathrm{E}+04$ & $-2 \mathrm{E}+03$ & $6 \mathrm{E}+02$ & $-2 \mathrm{E}+03$ & $-6 \mathrm{E}-06$ \\
$(0.5,0.999,0.5)$ & 1.000 & 2.000 & 3.000 & $1 \mathrm{E}-04$ & $-1 \mathrm{E}-04$ & $-8 \mathrm{E}-06$ & $-2 \mathrm{E}+06$ & $1 \mathrm{E}+07$ & $-2 \mathrm{E}+06$ & $6 \mathrm{E}+05$ & $-2 \mathrm{E}+06$ & $-6 \mathrm{E}-06$ \\
$(0.5,0.9999,0.5)$ & 1.000 & 2.000 & 3.000 & $1 \mathrm{E}-04$ & $-1 \mathrm{E}-04$ & $-8 \mathrm{E}-06$ & $-2 \mathrm{E}+09$ & $1 \mathrm{E}+10$ & $-2 \mathrm{E}+09$ & $6 \mathrm{E}+08$ & $-2 \mathrm{E}+09$ & $-6 \mathrm{E}-06$ \\
$(0.5,0.5,0.9)$ & 0.999 & 1.999 & 2.999 & $-2 \mathrm{E}-05$ & $-1 \mathrm{E}-04$ & $-7 \mathrm{E}-05$ & -5.773 & -4.677 & 31.909 & $-2 \mathrm{E}-05$ & -1.16 & $7 \mathrm{E}-01$ \\
$(0.5,0.5,0.99)$ & 0.999 & 1.999 & 2.999 & $-1 \mathrm{E}-05$ & $-1 \mathrm{E}-04$ & $1 \mathrm{E}-05$ & $-5 \mathrm{E}+03$ & $-5 \mathrm{E}+03$ & $3 \mathrm{E}+04$ & $-1 \mathrm{E}-05$ & $-9 \mathrm{E}+02$ & $6 \mathrm{E}+02$ \\
$(0.5,0.5,0.999)$ & 0.999 & 1.999 & 2.999 & $-9 \mathrm{E}-06$ & $-1 \mathrm{E}-04$ & $1 \mathrm{E}-05$ & $-5 \mathrm{E}+06$ & $-5 \mathrm{E}+06$ & $3 \mathrm{E}+07$ & $-1 \mathrm{E}-05$ & $-9 \mathrm{E}+05$ & $6 \mathrm{E}+05$ \\
$(0.5,0.5,0.9999)$ & 0.999 & 1.999 & 2.999 & $-9 \mathrm{E}-06$ & $-1 \mathrm{E}-04$ & $1 \mathrm{E}-05$ & $-5 \mathrm{E}+09$ & $-5 \mathrm{E}+09$ & $3 \mathrm{E}+10$ & $-1 \mathrm{E}-05$ & $-9 \mathrm{E}+08$ & $6 \mathrm{E}+08$ \\
\hline
\end{tabular}

Table 3.1: Interior point stress results for the cube under tensile stresses - Problem (A) 


\begin{tabular}{|c|c|c|c|c|c|c|c|c|c|c|c|c|}
\hline & \multicolumn{7}{|c|}{ With self-regularization } & \multicolumn{5}{c|}{ Without self-regularization } \\
\hline Coordinates & $\sigma_{11}$ & $\sigma_{22}$ & $\sigma_{33}$ & $\sigma_{12}$ & $\sigma_{23}$ & $\sigma_{13}$ & $\sigma_{11}$ & $\sigma_{22}$ & $\sigma_{33}$ & $\sigma_{12}$ & $\sigma_{23}$ & $\sigma_{13}$ \\
\hline$(0.5,0.5,0.5)$ & $-7 \mathrm{E}-07$ & $8 \mathrm{E}-07$ & $-4 \mathrm{E}-08$ & 1.000 & 1.000 & 1.000 & $-6 \mathrm{E}-07$ & $1 \mathrm{E}-06$ & $-1 \mathrm{E}-07$ & 0.999 & 0999 & 0999 \\
$(0.9,0.5,0.5)$ & $-8 \mathrm{E}-07$ & $9 \mathrm{E}-08$ & $-1 \mathrm{E}-06$ & 1.000 & 1.000 & 1.000 & 17.05 & -3.96 & -3.96 & -4.83 & 0.84 & -4.83 \\
$(0.99,0.5,0.5)$ & $-8 \mathrm{E}-07$ & $-6 \mathrm{E}-08$ & $-1 \mathrm{E}-06$ & 1.000 & 1.000 & 1.000 & $2 \mathrm{E}+04$ & $-3 \mathrm{E}+03$ & $-3 \mathrm{E}+03$ & $-5 \mathrm{E}+03$ & 0.54 & $-5 \mathrm{E}+03$ \\
$(0.999,0.5,0.5)$ & $-8 \mathrm{E}-07$ & $-5 \mathrm{E}-08$ & $-1 \mathrm{E}-06$ & 1.000 & 1.000 & 1.000 & $2 \mathrm{E}+07$ & $-3 \mathrm{E}+06$ & $-3 \mathrm{E}+06$ & $-5 \mathrm{E}+06$ & 0.50 & $-5 \mathrm{E}+06$ \\
$(0.9999,0.5,0.5)$ & $-8 \mathrm{E}-07$ & $-5 \mathrm{E}-08$ & $-1 \mathrm{E}-06$ & 1.000 & 1.000 & 1.000 & $2 \mathrm{E}+10$ & $-3 \mathrm{E}+09$ & $-3 \mathrm{E}+09$ & $-5 \mathrm{E}+09$ & 0.50 & $-5 \mathrm{E}+09$ \\
$(0.5,0.9,0.5)$ & $5 \mathrm{E}-07$ & $6 \mathrm{E}-07$ & $3 \mathrm{E}-07$ & 1.000 & 1.000 & 1.000 & $-4 \mathrm{E}+00$ & $2 \mathrm{E}+01$ & $-4 \mathrm{E}+00$ & $-5 \mathrm{E}+00$ & $-5 \mathrm{E}+00$ & $8 \mathrm{E}-01$ \\
$(0.5,0.99,0.5)$ & $1 \mathrm{E}-06$ & $4 \mathrm{E}-07$ & $6 \mathrm{E}-07$ & 1.000 & 1.000 & 1.000 & $-3 \mathrm{E}+03$ & $2 \mathrm{E}+04$ & $-3 \mathrm{E}+03$ & $-5 \mathrm{E}+03$ & $-5 \mathrm{E}+03$ & $5 \mathrm{E}-01$ \\
$(0.5,0.999,0.5)$ & $1 \mathrm{E}-06$ & $3 \mathrm{E}-07$ & $6 \mathrm{E}-07$ & 1.000 & 1.000 & 1.000 & $-3 \mathrm{E}+06$ & $2 \mathrm{E}+07$ & $-3 \mathrm{E}+06$ & $-5 \mathrm{E}+06$ & $-5 \mathrm{E}+06$ & $5 \mathrm{E}-01$ \\
$(0.5,0.9999,0.5)$ & $1 \mathrm{E}-06$ & $3 \mathrm{E}-07$ & $6 \mathrm{E}-07$ & 1.000 & 1.000 & 1.000 & $-3 \mathrm{E}+09$ & $2 \mathrm{E}+10$ & $-3 \mathrm{E}+09$ & $-5 \mathrm{E}+09$ & $-5 \mathrm{E}+09$ & $5 \mathrm{E}-01$ \\
$(0.5,0.5,0.9)$ & $-4 \mathrm{E}-07$ & $7 \mathrm{E}-07$ & $2 \mathrm{E}-07$ & 1.000 & 1.000 & 1.000 & $-4 \mathrm{E}+00$ & $-4 \mathrm{E}+00$ & $2 \mathrm{E}+01$ & $8 \mathrm{E}-01$ & $-5 \mathrm{E}+00$ & $-5 \mathrm{E}+00$ \\
$(0.5,0.5,0.99)$ & $9 \mathrm{E}-08$ & $8 \mathrm{E}-07$ & $8 \mathrm{E}-08$ & 1.000 & 1.000 & 1.000 & $-3 \mathrm{E}+03$ & $-3 \mathrm{E}+03$ & $2 \mathrm{E}+04$ & $5 \mathrm{E}-01$ & $-5 \mathrm{E}+03$ & $-5 \mathrm{E}+03$ \\
$(0.5,0.5,0.999)$ & $1 \mathrm{E}-07$ & $8 \mathrm{E}-07$ & $7 \mathrm{E}-08$ & 1.000 & 1.000 & 1.000 & $-3 \mathrm{E}+06$ & $-3 \mathrm{E}+06$ & $2 \mathrm{E}+07$ & $5 \mathrm{E}-01$ & $-5 \mathrm{E}+06$ & $-5 \mathrm{E}+06$ \\
$(0.5,0.5,0.9999)$ & $1 \mathrm{E}-07$ & $8 \mathrm{E}-07$ & $7 \mathrm{E}-08$ & 1.000 & 1.000 & 1.000 & $-3 \mathrm{E}+09$ & $-3 \mathrm{E}+09$ & $2 \mathrm{E}+10$ & $5 \mathrm{E}-01$ & $-5 \mathrm{E}+09$ & $-5 \mathrm{E}+09$ \\
\hline
\end{tabular}

Table 3.2: Interior point stress results for the cube under shear stresses - Problem (A) 


\begin{tabular}{|c|c|c|c|c|c|c|c|c|c|}
\hline & \multicolumn{5}{|c|}{$\mathrm{u}_{1}$} & \multicolumn{3}{c|}{$\mathrm{u}_{2}$} & \multicolumn{3}{c|}{$\mathrm{u}_{3}$} \\
\hline Coordinates & Exact & Self-reg. & No self-reg. & Exact & Self-reg. & No self-reg. & Exact & Self-reg. & No self-reg. \\
\hline$(0.5,0.5,0.5)$ & $2.500 \mathrm{E}-04$ & $-2.498 \mathrm{E}-04$ & $-2.498 \mathrm{E}-04$ & $4.000 \mathrm{E}-04$ & $3.999 \mathrm{E}-04$ & $3.999 \mathrm{E}-04$ & $1.050 \mathrm{E}-03$ & $1.045 \mathrm{E}-03$ & $1.050 \mathrm{E}-03$ \\
$(0.9,0.5,0.5)$ & $4.500 \mathrm{E}-04$ & $-4.496 \mathrm{E}-04$ & $-7.003 \mathrm{E}-04$ & $4.000 \mathrm{E}-04$ & $4.001 \mathrm{E}-04$ & $3.861 \mathrm{E}-04$ & $1.050 \mathrm{E}-03$ & $1.050 \mathrm{E}-03$ & $1.013 \mathrm{E}-03$ \\
$(0.99,0.5,0.5)$ & $4.950 \mathrm{E}-04$ & $-4.945 \mathrm{E}-04$ & $-4.163 \mathrm{E}-02$ & $4.000 \mathrm{E}-04$ & $4.001 \mathrm{E}-04$ & $4.193 \mathrm{E}-03$ & $1.050 \mathrm{E}-03$ & $1.050 \mathrm{E}-03$ & $1.100 \mathrm{E}-02$ \\
$(0.999,0.5,0.5)$ & $4.995 \mathrm{E}-04$ & $-4.990 \mathrm{E}-04$ & $-4.206 \mathrm{E}+00$ & $4.000 \mathrm{E}-04$ & $4.001 \mathrm{E}-04$ & $3.976 \mathrm{E}-01$ & $1.050 \mathrm{E}-03$ & $1.050 \mathrm{E}-03$ & $1.043 \mathrm{E}+00$ \\
$(0.9999,0.5,0.5)$ & $5.000 \mathrm{E}-04$ & $-4.995 \mathrm{E}-04$ & $-4.212 \mathrm{E}+02$ & $4.000 \mathrm{E}-04$ & $4.001 \mathrm{E}-04$ & $3.972 \mathrm{E}+01$ & $1.050 \mathrm{E}-03$ & $1.050 \mathrm{E}-03$ & $1.042 \mathrm{E}+02$ \\
$(0.5,0.9,0.5)$ & $2.500 \mathrm{E}-04$ & $-2.498 \mathrm{E}-04$ & $-2.411 \mathrm{E}-04$ & $7.200 \mathrm{E}-04$ & $7.199 \mathrm{E}-04$ & $1.139 \mathrm{E}-03$ & $1.050 \mathrm{E}-03$ & $1.050 \mathrm{E}-03$ & $1.013 \mathrm{E}-03$ \\
$(0.5,0.99,0.5)$ & $2.500 \mathrm{E}-04$ & $-2.498 \mathrm{E}-04$ & $-2.616 \mathrm{E}-03$ & $7.920 \mathrm{E}-04$ & $7.919 \mathrm{E}-04$ & $6.948 \mathrm{E}-02$ & $1.050 \mathrm{E}-03$ & $1.050 \mathrm{E}-03$ & $1.100 \mathrm{E}-02$ \\
$(0.5,0.999,0.5)$ & $2.500 \mathrm{E}-04$ & $-2.498 \mathrm{E}-04$ & $-2.481 \mathrm{E}-01$ & $7.992 \mathrm{E}-04$ & $7.991 \mathrm{E}-04$ & $6.769 \mathrm{E}+00$ & $1.050 \mathrm{E}-03$ & $1.050 \mathrm{E}-03$ & $1.043 \mathrm{E}+00$ \\
$(0.5,0.9999,0.5)$ & $2.500 \mathrm{E}-04$ & $-2.498 \mathrm{E}-04$ & $-2.479 \mathrm{E}+01$ & $7.999 \mathrm{E}-04$ & $7.998 \mathrm{E}-04$ & $6.750 \mathrm{E}+02$ & $1.050 \mathrm{E}-03$ & $1.050 \mathrm{E}-03$ & $1.042 \mathrm{E}+02$ \\
$(0.5,0.5,0.9)$ & $2.500 \mathrm{E}-04$ & $-2.500 \mathrm{E}-04$ & $-2.413 \mathrm{E}-04$ & $4.000 \mathrm{E}-04$ & $3.998 \mathrm{E}-04$ & $3.858 \mathrm{E}-04$ & $1.890 \mathrm{E}-03$ & $1.889 \mathrm{E}-03$ & $2.979 \mathrm{E}-03$ \\
$(0.5,0.5,0.99)$ & $2.500 \mathrm{E}-04$ & $-2.500 \mathrm{E}-04$ & $-2.619 \mathrm{E}-03$ & $4.000 \mathrm{E}-04$ & $3.997 \mathrm{E}-04$ & $4.187 \mathrm{E}-03$ & $2.079 \mathrm{E}-03$ & $2.078 \mathrm{E}-03$ & $1.806 \mathrm{E}-01$ \\
$(0.5,0.5,0.999)$ & $2.500 \mathrm{E}-04$ & $-2.500 \mathrm{E}-04$ & $-2.484 \mathrm{E}-01$ & $4.000 \mathrm{E}-04$ & $3.997 \mathrm{E}-04$ & $3.970 \mathrm{E}-01$ & $2.098 \mathrm{E}-03$ & $2.097 \mathrm{E}-03$ & $1.774 \mathrm{E}+01$ \\
$(0.5,0.5,0.9999)$ & $2.500 \mathrm{E}-04$ & $-2.500 \mathrm{E}-04$ & $-2.482 \mathrm{E}+01$ & $4.000 \mathrm{E}-04$ & $3.997 \mathrm{E}-04$ & $3.967 \mathrm{E}+01$ & $2.100 \mathrm{E}-03$ & $2.099 \mathrm{E}-03$ & $1.771 \mathrm{E}+03$ \\
\hline
\end{tabular}

Table 3.3: Interior point displacement results for the cube under tensile stresses - Problem (A) 


\begin{tabular}{|c|c|c|c|c|c|c|c|c|c|}
\hline & \multicolumn{3}{|c|}{$\mathrm{u}_{1}$} & \multicolumn{3}{c|}{$\mathrm{u}_{2}$} & \multicolumn{3}{c|}{$\mathrm{u}_{3}$} \\
\hline Coordinates & Exact & Self-reg. & No self-reg. & Exact & Self-reg. & No self-reg. & Exact & Self-reg. & No self-reg. \\
\hline$(0.5,0.5,0.5)$ & $1.300 \mathrm{E}-03$ & $1.302 \mathrm{E}-03$ & $1.302 \mathrm{E}-03$ & $1.300 \mathrm{E}-03$ & $1.297 \mathrm{E}-03$ & $1.297 \mathrm{E}-03$ & $1.300 \mathrm{E}-03$ & $1.299 \mathrm{E}-03$ & $1.299 \mathrm{E}-03$ \\
$(0.9,0.5,0.5)$ & $1.300 \mathrm{E}-03$ & $1.302 \mathrm{E}-03$ & $1.942 \mathrm{E}-03$ & $1.820 \mathrm{E}-03$ & $1.817 \mathrm{E}-03$ & $1.744 \mathrm{E}-03$ & $1.820 \mathrm{E}-03$ & $1.817 \mathrm{E}-03$ & $1.744 \mathrm{E}-03$ \\
$(0.99,0.5,0.5)$ & $1.300 \mathrm{E}-03$ & $1.302 \mathrm{E}-03$ & $1.106 \mathrm{E}-01$ & $1.937 \mathrm{E}-03$ & $1.934 \mathrm{E}-03$ & $2.085 \mathrm{E}-02$ & $1.937 \mathrm{E}-03$ & $1.934 \mathrm{E}-03$ & $2.085 \mathrm{E}-02$ \\
$(0.999,0.5,0.5)$ & $1.300 \mathrm{E}-03$ & $1.302 \mathrm{E}-03$ & $1.099 \mathrm{E}+01$ & $1.949 \mathrm{E}-03$ & $1.945 \mathrm{E}-03$ & $1.940 \mathrm{E}+00$ & $1.949 \mathrm{E}-03$ & $1.945 \mathrm{E}-03$ & $1.940 \mathrm{E}+00$ \\
$(0.9999,0.5,0.5)$ & $1.300 \mathrm{E}-03$ & $1.302 \mathrm{E}-03$ & $1.099 \mathrm{E}+03$ & $1.950 \mathrm{E}-03$ & $1.947 \mathrm{E}-03$ & $1.933 \mathrm{E}+02$ & $1.950 \mathrm{E}-03$ & $1.947 \mathrm{E}-03$ & $1.933 \mathrm{E}+02$ \\
$(0.5,0.9,0.5)$ & $1.820 \mathrm{E}-03$ & $1.823 \mathrm{E}-03$ & $1.749 \mathrm{E}-03$ & $1.300 \mathrm{E}-03$ & $1.297 \mathrm{E}-03$ & $1.934 \mathrm{E}-03$ & $1.820 \mathrm{E}-03$ & $1.821 \mathrm{E}-03$ & $1.748 \mathrm{E}-03$ \\
$(0.5,0.99,0.5)$ & $1.937 \mathrm{E}-03$ & $1.940 \mathrm{E}-03$ & $2.091 \mathrm{E}-02$ & $1.300 \mathrm{E}-03$ & $1.297 \mathrm{E}-03$ & $1.101 \mathrm{E}-01$ & $1.937 \mathrm{E}-03$ & $1.939 \mathrm{E}-03$ & $2.090 \mathrm{E}-02$ \\
$(0.5,0.999,0.5)$ & $1.949 \mathrm{E}-03$ & $1.951 \mathrm{E}-03$ & $1.946 \mathrm{E}+00$ & $1.300 \mathrm{E}-03$ & $1.297 \mathrm{E}-03$ & $1.095 \mathrm{E}+01$ & $1.949 \mathrm{E}-03$ & $1.950 \mathrm{E}-03$ & $1.945 \mathrm{E}+00$ \\
$(0.5,0.9999,0.5)$ & $1.950 \mathrm{E}-03$ & $1.953 \mathrm{E}-03$ & $1.939 \mathrm{E}+02$ & $1.300 \mathrm{E}-03$ & $1.297 \mathrm{E}-03$ & $1.094 \mathrm{E}+03$ & $1.950 \mathrm{E}-03$ & $1.952 \mathrm{E}-03$ & $1.938 \mathrm{E}+02$ \\
$(0.5,0.5,0.9)$ & $1.820 \mathrm{E}-03$ & $1.825 \mathrm{E}-03$ & $1.751 \mathrm{E}-03$ & $1.820 \mathrm{E}-03$ & $1.815 \mathrm{E}-03$ & $1.742 \mathrm{E}-03$ & $1.300 \mathrm{E}-03$ & $1.299 \mathrm{E}-03$ & $1.938 \mathrm{E}-03$ \\
$(0.5,0.5,0.99)$ & $1.937 \mathrm{E}-03$ & $1.942 \mathrm{E}-03$ & $2.093 \mathrm{E}-02$ & $1.937 \mathrm{E}-03$ & $1.932 \mathrm{E}-03$ & $2.082 \mathrm{E}-02$ & $1.300 \mathrm{E}-03$ & $1.299 \mathrm{E}-03$ & $1.103 \mathrm{E}-01$ \\
$(0.5,0.5,0.999)$ & $1.949 \mathrm{E}-03$ & $1.954 \mathrm{E}-03$ & $1.948 \mathrm{E}+00$ & $1.949 \mathrm{E}-03$ & $1.943 \mathrm{E}-03$ & $1.938 \mathrm{E}+00$ & $1.300 \mathrm{E}-03$ & $1.299 \mathrm{E}-03$ & $1.097 \mathrm{E}+01$ \\
$(0.5,0.5,0.9999)$ & $1.950 \mathrm{E}-03$ & $1.955 \mathrm{E}-03$ & $1.941 \mathrm{E}+02$ & $1.950 \mathrm{E}-03$ & $1.944 \mathrm{E}-03$ & $1.931 \mathrm{E}+02$ & $1.300 \mathrm{E}-03$ & $1.299 \mathrm{E}-03$ & $1.096 \mathrm{E}+03$ \\
\hline
\end{tabular}

Table 3.4: Interior point displacement results for the cube under shear stresses - Problem (A) 


\begin{tabular}{|c|c|c|c|c|c|}
\hline \multicolumn{6}{|c|}{ Axial stresses } \\
\hline $\mathrm{y}$ & Exact & Self-reg. & Error \% & Without self-reg. & Error \% \\
\hline 0.4999 & -29.994 & -29.640 & -1.180 & -7879.0 & 26168.6 \\
\hline 0.499 & -29.940 & -29.586 & -1.182 & -7791.4 & 25923.4 \\
\hline 0.49 & -29.400 & -28.804 & -2.027 & -1537.3 & 5128.9 \\
\hline 0.4 & -24.000 & -23.538 & -1.925 & -103.7 & 332.17 \\
\hline 0.2 & -12.000 & -11.786 & -1.783 & -11.875 & -1.042 \\
\hline 0 & 0.000 & $9.054 \mathrm{E}-05$ & - & $4.9 \mathrm{E}-05$ & - \\
\hline-0.2 & 12.000 & 11.786 & -1.783 & 11.875 & -1.042 \\
\hline-0.4 & 24.000 & 23.539 & -1.921 & 103.72 & 332.17 \\
\hline-0.49 & 29.400 & 28.805 & -2.024 & 1537.3 & 5128.9 \\
\hline-0.499 & 29.940 & 29.587 & -1.179 & 7791.4 & 25923.4 \\
\hline-0.4999 & 29.994 & 29.641 & -1.177 & 7879.0 & 26168.6 \\
\hline \multicolumn{6}{|c|}{ Vertical displacements } \\
\hline $\mathrm{y}$ & Exact & Self-reg. & Error \% & Without self-reg. & Error \% \\
\hline 0.4999 & 0.378 & 0.380 & 0.529 & 0.191 & -49.5 \\
\hline 0.499 & 0.378 & 0.380 & 0.529 & 0.197 & -47.8 \\
\hline 0.49 & 0.378 & 0.380 & 0.529 & 0.269 & -28.7 \\
\hline 0.4 & 0.378 & 0.380 & 0.529 & 0.381 & 0.79 \\
\hline 0.2 & 0.378 & 0.380 & 0.529 & 0.380 & 0.529 \\
\hline 0 & 0.378 & 0.380 & 0.529 & 0.380 & 0.529 \\
\hline-0.2 & 0.378 & 0.380 & 0.529 & 0.380 & 0.529 \\
\hline-0.4 & 0.378 & 0.380 & 0.529 & 0.381 & 0.79 \\
\hline-0.49 & 0.378 & 0.380 & 0.529 & 0.269 & -28.7 \\
\hline-0.499 & 0.378 & 0.380 & 0.529 & 0.197 & -47.8 \\
\hline-0.4999 & 0.378 & 0.380 & 0.529 & 0.191 & -49.5 \\
\hline
\end{tabular}

Table 3.5: Results for the cantilever beam - Problem (B) 


\begin{tabular}{|c||c|c|c|c|c|c|c|c|c|}
\hline \multicolumn{1}{|c||}{} & \multicolumn{3}{c|}{$\sigma_{\theta}$} & \multicolumn{3}{c|}{$\sigma_{r}$} & \multicolumn{3}{c|}{$\mathrm{u}_{r}$} \\
\hline $\mathrm{r}$ & Self-reg. & Exact & Error \% & Self-reg. & Exact & Error \% & Self-reg. & Exact & Error $\%$ \\
\hline 1.0001 & 1.6537 & 1.6664 & -0.76 & -1.0035 & -0.9997 & 0.38 & $1.905 \mathrm{E}-03$ & $1.907 \mathrm{E}-03$ & -0.0895 \\
1.001 & 1.6525 & 1.6640 & -0.69 & -1.0023 & -0.9973 & 0.49 & $1.903 \mathrm{E}-03$ & $1.905 \mathrm{E}-03$ & -0.0902 \\
1.01 & 1.6413 & 1.6404 & 0.05 & -0.9891 & -0.9737 & 1.58 & $1.889 \mathrm{E}-03$ & $1.891 \mathrm{E}-03$ & -0.0978 \\
1.1 & 1.4510 & 1.4353 & 1.09 & -0.7560 & -0.7686 & -1.63 & $1.762 \mathrm{E}-03$ & $1.766 \mathrm{E}-03$ & -0.2442 \\
1.5 & 0.9254 & 0.9259 & -0.06 & -0.2570 & -0.2593 & -0.88 & $1.414 \mathrm{E}-03$ & $1.416 \mathrm{E}-03$ & -0.1348 \\
1.9 & 0.6873 & 0.7027 & -2.19 & -0.0308 & -0.0360 & -14.50 & $1.240 \mathrm{E}-03$ & $1.242 \mathrm{E}-03$ & -0.1690 \\
1.99 & 0.6630 & 0.6700 & -1.06 & -0.0004 & -0.0034 & -87.20 & $1.215 \mathrm{E}-03$ & $1.216 \mathrm{E}-03$ & -0.1000 \\
1.999 & 0.6615 & 0.6670 & -0.82 & 0.0012 & -0.0003 & -44.74 & $1.212 \mathrm{E}-03$ & $1.214 \mathrm{E}-03$ & -0.0979 \\
1.9999 & 0.6614 & 0.6667 & -0.80 & 0.0013 & $-3.3 \mathrm{E}-05$ & -3969.71 & $1.212 \mathrm{E}-03$ & $1.213 \mathrm{E}-03$ & -0.0973 \\
\hline
\end{tabular}

Table 3.6: Results of the internally pressurized cylinder - Problem (C) 


\begin{tabular}{|c||c|c|c|c|c|c|}
\hline \multicolumn{1}{|c||}{} & \multicolumn{3}{c|}{$\sigma_{1}$} & \multicolumn{3}{c|}{$\sigma_{2}$} \\
\hline $\mathrm{r}$ & Exact & Self-reg. & Error \% & Exact & Self-reg. & Error \% \\
\hline 2.001 & 1.131 & 1.121 & -0.886 & 1.131 & 1.099 & -2.761 \\
2.01 & 1.121 & 1.115 & -0.502 & 1.121 & 1.095 & -2.366 \\
2.1 & 1.035 & 1.046 & 1.060 & 1.035 & 1.038 & 0.316 \\
2.5 & 0.785 & 0.792 & 0.925 & 0.785 & 0.790 & 0.593 \\
2.9 & 0.654 & 0.653 & -0.217 & 0.654 & 0.648 & -0.996 \\
2.99 & 0.634 & 0.641 & 1.105 & 0.634 & 0.634 & 0.063 \\
2.999 & 0.632 & 0.640 & 1.236 & 0.632 & 0.633 & 0.192 \\
\hline \hline \multicolumn{1}{|||}{} & \multicolumn{2}{|c|}{$\sigma_{3}$} & & & $\mathrm{u}_{\tau}$ & \\
\hline $\mathrm{r}$ & Exact & Self-reg. & Error \% & Exact & Self-reg. & Error \% \\
\hline 2.001 & -0.998 & -0.980 & -1.831 & $2.183 \mathrm{E}-03$ & $2.180 \mathrm{E}-03$ & -0.112 \\
2.01 & -0.979 & -0.969 & -1.031 & $2.168 \mathrm{E}-03$ & $2.164 \mathrm{E}-03$ & -0.171 \\
2.1 & -0.807 & -0.812 & 0.694 & $2.029 \mathrm{E}-03$ & $2.025 \mathrm{E}-03$ & -0.221 \\
2.5 & -0.307 & -0.326 & 6.190 & $1.603 \mathrm{E}-03$ & $1.598 \mathrm{E}-03$ & -0.323 \\
2.9 & -0.045 & -0.040 & -10.596 & $1.367 \mathrm{E}-03$ & $1.364 \mathrm{E}-03$ & -0.205 \\
2.99 & -0.004 & -0.008 & 91.094 & $1.330 \mathrm{E}-03$ & $1.328 \mathrm{E}-03$ & -0.193 \\
2.999 & 0.000 & -0.006 & 1276.582 & $1.327 \mathrm{E}-03$ & $1.324 \mathrm{E}-03$ & -0.225 \\
\hline
\end{tabular}

Table 3.7: Results of the internally pressurized sphere - Problem (C) 


\begin{tabular}{|c|c|c|}
\hline & $\sigma_{1}$ & Error $\%$ \\
\hline Analytical(Pilkey 2008) & 2.185 & - \\
\hline BEM boundary solution & 2.2217 & 1.67 \\
\hline BEM interior point solution & 2.1803 & 0.215 \\
\hline FEM & 2.2368 & 2.37 \\
\hline
\end{tabular}

Table 3.8: Stress concentration factors obtained from BEM and FEM solutions Problem (D-2) 


\section{Chapter 4}

\section{Self-regularized Traction-BIE}

\subsection{Introduction}

In this chapter, the self-regularization technique for interior points in Chapter 3 is extended to obtain a traction-BIE by taking the interior point to the boundary. This scheme was proposed by Richardson and Cruse (1999) in two dimensions and will be again extended to three dimensions in this study. Two approaches to treat the stress continuity issue, as discussed in Chapter 2, are employed, and are described in more detail. Numerical examples are given after the analytical treatment in which results from traction-BIE and displacement-BIE are compared.

\subsection{Self-regularized Traction-BIE}

In Equation (3.4), the stresses at an interior point $p$ are regularized by the constant stress state terms at the regularization point $P$ on the boundary. If the interior point is taken to the boundary so that $p$ and $P$ coincide, Equation (3.4) becomes:

$0=-\int_{S}\left[u_{k}(Q)-u_{k}^{L}(Q)\right] S_{k i j}(P, Q) n_{i}(P) d S(Q)+\int_{S}\left[t_{k}(Q)-t_{k}^{L}(Q)\right] D_{k i j}(P, Q) n_{i}(P) d S(Q)$ 
where $u_{k}^{L}(Q)$ and $t_{k}^{L}(Q)$ are given in Equations (3.5) and (3.6), respectively, and the outward normal $n_{i}(P)$ is used to reduce the redundancy produced by the index $i$. Numerical evaluations of the integrals will no longer be an issue since they are fully regularized. The process of sub-division of elements employed in displacement-BIE when evaluating singular integrals is therefore eliminated.

The constant stress terms $u_{k}^{L}(Q)$ and $t_{k}^{L}(Q)$ need to be expressed by the variables on the surface nodes so that the corresponding coefficients of these terms can be put into the system matrix. Following the same steps as for $2 \mathrm{D}$ described in Richardson and Cruse (1999), the displacement gradients at $P$ can be expressed by nodal tractions and displacements for 3D as:

$$
u_{k, m}(P)=A_{k m r} t_{r}\left(\xi_{1}^{P}, \xi_{2}^{P}\right)+B_{k m r} \sum_{i=1}^{8 \text { or } 6} N_{i}^{\prime} u_{r}^{i}\left(\xi_{1}^{P}, \xi_{2}^{P}\right)
$$

where $A_{k m r}$ contains the first three rows of $\left[\mathbf{A}^{*}\right]^{-1}$ and $B_{k m r}$ contains the last six rows, and $N_{i}^{\prime}$ are the derivatives of shape functions at local coordinates $\xi_{1}, \xi_{2}$ corresponding 
to $P$. The constant stress displacements $u_{k}^{L}(Q)$ can now be expressed as

$u_{k}^{L}(Q)=u_{k}(P)+[\mathbf{D}]\left[\mathbf{A}^{*}\right]^{-1}\left\{\begin{array}{c}t_{1} \\ t_{2} \\ t_{3} \\ \frac{u_{1}}{\xi_{1}} \\ \frac{u_{2}}{\xi_{1}} \\ \frac{u_{3}}{\xi_{1}} \\ \frac{u_{1}}{\xi_{2}} \\ \frac{u_{2}}{\xi_{2}} \\ \frac{u_{3}}{\xi_{2}}\end{array}\right\}=u_{k}(P)+C_{k m r} t_{r}\left(\xi_{1}^{P}, \xi_{2}^{P}\right)+D_{k m r} \sum_{i=1}^{8 r} N_{i}^{\prime} u_{r}^{i}\left(\xi_{1}^{P}, \xi_{2}^{P}\right)$

where $C_{k m r}$ and $D_{k m r}$ contain first three rows and subsequent six rows, respectively, of product of the matrices $[\mathbf{D}]$ and $\left[\mathbf{A}^{*}\right]^{-1}$. The matrix $[\mathbf{D}]$ contains the terms $\left[x_{m}(Q)-\right.$ $x_{m}(P)$, and is shown in Equation (4.4). Similarly, the constant stress tractions $t_{k}^{L}(Q)$ are expressed by multiplying the matrix $[\mathbf{S}]$ in Equation (4.5) to the inverse matrix of $\left[\mathbf{A}^{*}\right]$. 


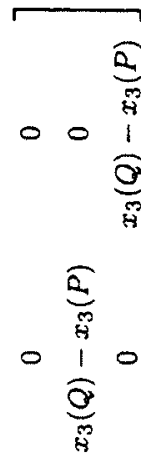

खृ

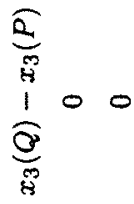

$00 \frac{\substack{\mathbb{E} \\ \frac{\pi}{\mathbb{G}}}}{1}$

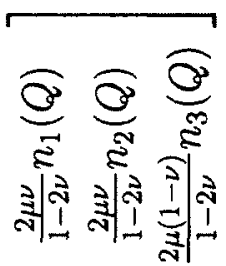

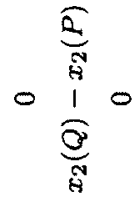

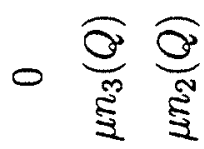

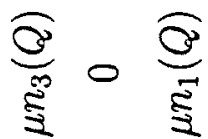

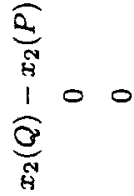

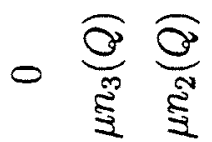

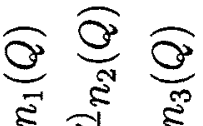

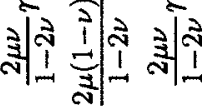

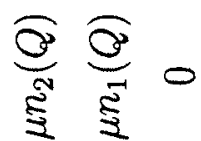

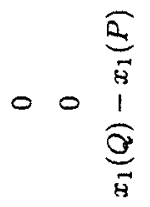

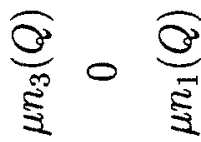

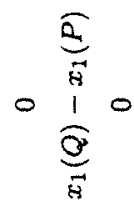

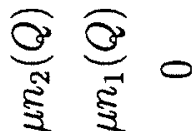
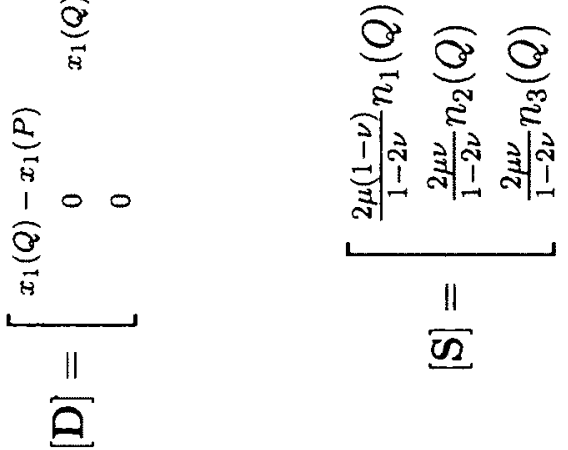
Then $t_{k}^{L}(Q)$ can be expressed by nodal values as:

$$
t_{k}^{L}(Q)=[\mathbf{S}]\left[\mathbf{A}^{*}\right]^{-1}\left\{\begin{array}{c}
t_{1} \\
t_{2} \\
t_{3} \\
\frac{u_{1}}{\xi_{1}} \\
\frac{u_{2}}{\xi_{1}} \\
\frac{u_{3}}{\xi_{1}} \\
\frac{u_{1}}{\xi_{2}} \\
\frac{u_{2}}{\xi_{2}} \\
\frac{u_{3}}{\xi_{2}}
\end{array}\right\}=E_{k m r} t_{r}\left(\xi_{1}^{P}, \xi_{2}^{P}\right)+F_{k m r} \sum_{i=1}^{8} N_{i}^{\prime} u_{r}^{i}\left(\xi_{1}^{P}, \xi_{2}^{P}\right)
$$

where $E_{k m r}$ contains the first three rows of the product of $[\mathbf{S}]$ and $\left[\mathbf{A}^{*}\right]^{-1}$, and $F_{k m r}$ contains the remaining six rows. Substituting Equations (4.3) and (4.6) into Equation (4.1) and rearranging the coefficients, the following expression is obtained:

$$
\begin{aligned}
& \int_{S}\left[u_{k}(Q) S_{k i j}(P, Q)-u_{k}(P) S_{k i j}(P, Q)\right] n_{i}(P) d S \\
+ & \int_{S}\left[F_{k m r} D_{k i j}(P, Q)-D_{k m r} S_{k i j}(P, Q)\right] N^{\prime}\left(\xi_{1}^{P}, \xi_{2}^{P}\right) n_{i}(P) u_{k}\left(P^{M}\right) d S \\
= & \int_{S} t_{k}(Q) D_{k i j}(P, Q) d S+\int_{S}\left[C_{k m r} S_{k i j}(P, Q)-E_{k m r} D_{k i j}(P, Q)\right] n_{i}(P) t_{k}\left(P^{M}\right) d S
\end{aligned}
$$

The symbols $u_{k}\left(P^{M}\right)$ and $t_{k}\left(P^{M}\right)$ denote that their coefficients will be put into the matrix depending on the element $M$ that contains the regularization point $P$. This 
will be discussed in the next section as this element will be taken differently by the two approaches in the attempt to satisfy displacement-gradient continuity. The coefficients on the left-hand side of Equation (4.7) will be stored in matrix $[\mathbf{A}]$ in Equation (2.21) while coefficients on the right-hand side are stored in matrix [B]. The singularities of the kernels are no longer of concern as they are fully regularized. The regularization is done before discretization so that the same mesh can be used as in displacement-BIE. It is evident from the above, however, that the matrix operations are significantly more involved in the $3 \mathrm{D}$ case here as compared to those in $2 \mathrm{D}$.

With the quadratic elements used, the displacement gradients required in Equation (4.1), as well as the stresses, are linearly distributed within the elements. However, there could be a discontinuity across the element boundaries. As briefly described in Chapter 2, several analytical schemes have been proposed to solve this problem, but are not easy to implement, efficient nor practical in three dimensions due to their complexity. Two strategies, namely, the relaxed continuity method, proposed by Richardson and Cruse (1999), and the collocation strategy by Gallego and Dominguez (1996) are adopted in this study and will be described in detail in the next section.

\subsection{Treatments of Displacement-gradient Discon- tinuity}

\subsubsection{Relaxed Continuity}

In Richardson and Cruse (1999), the boundary point $P$ becomes a boundary node after discretization. The displacement gradients thus would be discontinuous from 
one element to another sharing the node. It was suggested that the displacement gradients in Equation (4.3) can be expressed by the average of each of respective values from the elements sharing the node, i.e.,

$$
u_{k}^{L}(Q)= \begin{cases}u_{k}^{P}+u_{k, m}\left(P^{\Delta S}\right)\left[x_{m}(Q)-x_{m}(P)\right] & \text { for } P \in \Delta S \\ u_{k}^{P}+\left[\frac{1}{M} \sum_{J=1}^{M} u_{k, m}\left(P^{J}\right)\right]\left[x_{m}(Q)-x_{m}(P)\right] & \text { for } P \notin \Delta S\end{cases}
$$

In Equation (4.8), $M$ is the number of elements sharing the node $P, \Delta S$ the element being integrated, and the displacement gradients $u_{k, m}\left(P^{J}\right)$ are expressed by the nodal tractions and displacements that belong to the $J$-th element. When $\Delta S$ is one of the $M$ elements, no average is taken but only nodal values belonging to $\Delta S$ are involved.

\subsubsection{Collocation Strategy}

The collocation strategy moves the collocation point into the interior of the elements where the continuity requirement is satisfied, instead of at the boundary nodes. The intrinsic coordinates of the collocation points are set as, for example, $\xi_{1}, \xi_{2}= \pm 0.9$ rather than \pm 1 for quadrilateral elements, as illustrated in Figure 4.1. This approach is different from the use of discontinuous elements which form a much larger system matrix since the number of collocation points are much greater than the number of boundary nodes. In the present approach, the displacement gradients at the collocation point inside an element, and consequently the constant stress state displacements and tractions, are expressed in terms of the boundary values of the element containing the collocation point. For each node shared by $M$ elements, there will be $M$ collocation points around it, each producing one set of 3 equations. Thus for each node, there will be $M$ set of equations written onto it to yield only one set of equations, as when continuous elements are used. This is known as the Multiple Collocation Approach 
(MCA) (Dominguez et al. 2000). This scheme will be applied to the whole surface of the body instead of partial application as in Dominguez et al. (2000), Dominguez and Ariza (2000).

\subsection{Numerical Examples}

In this section, the self-regularized traction-BIE is implemented for some threedimensional elasticity problems. Both the relaxed continuity approach and collocation strategy are tested. For the collocation method, a study was conducted first to find the best location of the collocation points inside the elements. This was established to be at $\xi_{1}, \xi_{2}= \pm 0.9$ for most cases. This location will be used for all the following numerical examples. The calculated boundary values, along with those obtained from conventional displacement-BIE, are compared with the exact solutions.

\subsubsection{Thin Plate under Uniform Stress}

The first example is a rectangular plate whose material properties are the same as those used in Chapter 3. It is subject to uniform stresses of $\sigma_{11}=1, \sigma_{22}=2, \sigma_{33}=3$. The dimensions in $x_{1}$ and $x_{2}$ directions are taken to be unity, while the thickness in $x_{3}$ direction is gradually decreased from 1 to 0.01 to form a thin plate. Both relaxed continuity and collocation strategy are employed. Figures 4.2 and 4.3 show the maximum errors in stresses and displacement, respectively, obtained from both traction-BIE and displacement-BIE against the thickness.

As can be seen in the results, the traction-BIE combined with collocation method provides very accurate results, while those with the relaxed continuity start to deteriorate slightly when the thickness reduces to 0.01 . In comparison, the conventional displacement-BIE starts to fail when the thickness decreases to 0.3 . 
The collocation method in conjunction with traction-BIE thus provides a possible solution to this thin plate problem under uniform stresses.

\subsubsection{Thick-walled Cylinder under Axial Load and Internal Pressure}

The second example is a hollow cylinder with a radius ratio of 2 subject to axial loading and internal pressure. A $\frac{1}{4}$-model is first tested; the relatively coarse mesh used is shown in Figure 4.4. A series of nodes along two vertical lines on the inner and outer surfaces are observed, whose vertical location range from 0 to 1 with 1 being the total height. For the axial loading case, both approaches give very good results, as shown in Table 4.1. This is due to the applied uniform stress which has no discontinuity issues, as also seen above in the thin plate example. For the internal pressure case, however, the relaxed continuity approach was found to produce severe erroneous results, possibly caused by discontinuity at the corners; these results are not presented here. The results at these nodes obtained from the collocation method and displacement-BIE are shown in Table 4.2. This method better satisfies continuity at the corners, since collocation points are always within elements. However, the results are still not uniformly accurate, and contain more than $10 \%$ errors at some nodes. This is, again, caused by the presence of the corners in the model. Another fullymodeled cylinder is then tested, as shown in Figure 4.5, to reduce the effect of corners. The results are listed in Table 4.3 , which are somewhat better than the $\frac{1}{4}$-model, but the maximum errors are still relatively high. This suggests that neither of these two methods can sufficiently ensure displacement-gradient continuity across element boundaries, especially at the corners. No further tests on a thin-walled cylinder are therefore conducted. 


\subsubsection{Internally Pressurized Sphere}

The final example is a hollow sphere under internal pressure, whose dimensions and material properties are the same as in Chapter 3. A fully-modeled sphere is used instead of the $\frac{1}{8}$-model in Chapter 3 to avoid the corners. The boundary element mesh is shown in Figure 4.6, with 48 elements and 148 nodes. Figures 4.7 and 4.8 present the variation of maximum error in stresses and displacements, respectively, with the radius ratio $K$ ranging from 1.1 to 2 .

The results show that with the absence of corners, the traction-BIE can provide even better results (less than $2 \%$ error) than the displacement-BIE (3.3\% error) when $K$ is high, with either relaxed continuity or collocation strategy. When the thickness of the hollow sphere reduces, however, the traction-BIE starts to fail at $K=1.4$ while displacement-BIE gives good results until $K=1.1$. Refinement of the mesh for small thicknesses could provide better accuracy but this would defeat any general purpose for using the traction-BIE instead of commonly used displacement-BIE since the latter can give good results with slightly refined mesh. Indeed, no convergence of the results is observed with mesh refinement with the traction-BIE in this case. This suggests that when stresses are not uniformly distributed within the body, the traction-BIE is not able to recover good results for the thin-walled sphere.

\subsubsection{Discussion}

The numerical examples shown in the previous section exposed the problems which arise when using the self-regularized traction-BIE. When treating problems with uniform stresses, the traction-BIE gives excellent results even for very thin-body problems, since no stress-discontinuity is involved. For more general cases, the two 
approaches for eliminating stress-discontinuity in the formulation only works when no corner is present. When corners are involved, the displacement gradients and stresses cannot be ensured to be continuous at the nodes there across the elements. This causes the deterioration of the results. The self-regularized traction-BIE is thus not well-suited for general three-dimensional problems unless some other methods, which are analytically and numerically simple, can be developed to treat the displacement-gradient discontinuity issue.

Contrary to what is suggested in Richardson and Cruse (1999), the proposed self-regularized traction-BIE, unfortunately, does not seem well-suited for thin-body problems either. The reason of this deficiency can be explained as follows: when the integration point $Q$ shares the same element with the source point $P$, the singularities of the kernels are cancelled by the constant stress terms calculated at $P$, i.e., when the regularization point coincides with the source point itself. However, when dealing with thin-body problems, another surface would approach $P$. This is illustrated in Figure 4.9 where an interior point lies between boundaries $S_{a}$ and $S_{b}$, but its regularization point will be located on $S_{a}$ since it is closer. If the boundary $S_{b}$ is close to $p$, the singularities in the integrals over a field point $Q^{\prime}$ on $S_{b}$ cannot be regularized by the constant stress terms on point $P$. In Equations (3.4) and (4.1), the regularization terms are obtained by applying simple solutions to the problem being treated, thus only one regularization point can be chosen. Therefore, if two surfaces are both close to interior point $p$, only integrals on one surface are regularized, leaving those on the other surface near-singular. For this reason, when $p$ is taken to $P$ to form the traction-BIE, it is still not well-suited for solving thin-body problem unless some other special regularization scheme is used to reduce the near-singularities due to the presence of the second near surface. 


\subsection{Summary}

In this chapter, the self-regularized traction-BIE is formed by taking an interior point to the boundary using the self-regularization scheme for Somigliana's stress identity in Chapter 3. Two approaches, i.e. the relaxed continuity approach and the collocation strategy, were tested in attempt to treat the discontinuity in displacement gradients that occur at inter-element boundaries when using conforming isoparametric quadratic elements. Several numerical examples were presented. For problems subject to uniform stresses, the traction-BIE can provide excellent results with both continuity treatments, as well as treating thin-body problems. However, for more general cases where stresses are not uniform and in the presence of geometric corners, the traction-BIE fails to give consistently good results. When no corners are involved in a non-slender body, the traction-BIE combined with the two methods can provide better results than the conventional displacement-BIE. For thin-body problems, the present traction-BIE fails again due to the fact that near-singularities at the second near surface are not regularized. This is contrary to the statement in Richardson and Cruse (1999) that the whole-body regularization should be well suited for thin-body problems.

Inspired by the simplicity of the numerical implementation of the self-regularization scheme, and the analysis in the previous section showing the failure of the traction-BIE to solve thin-body problem, a new scheme is proposed using the displacement-BIE. This will be discussed in the next chapter. 


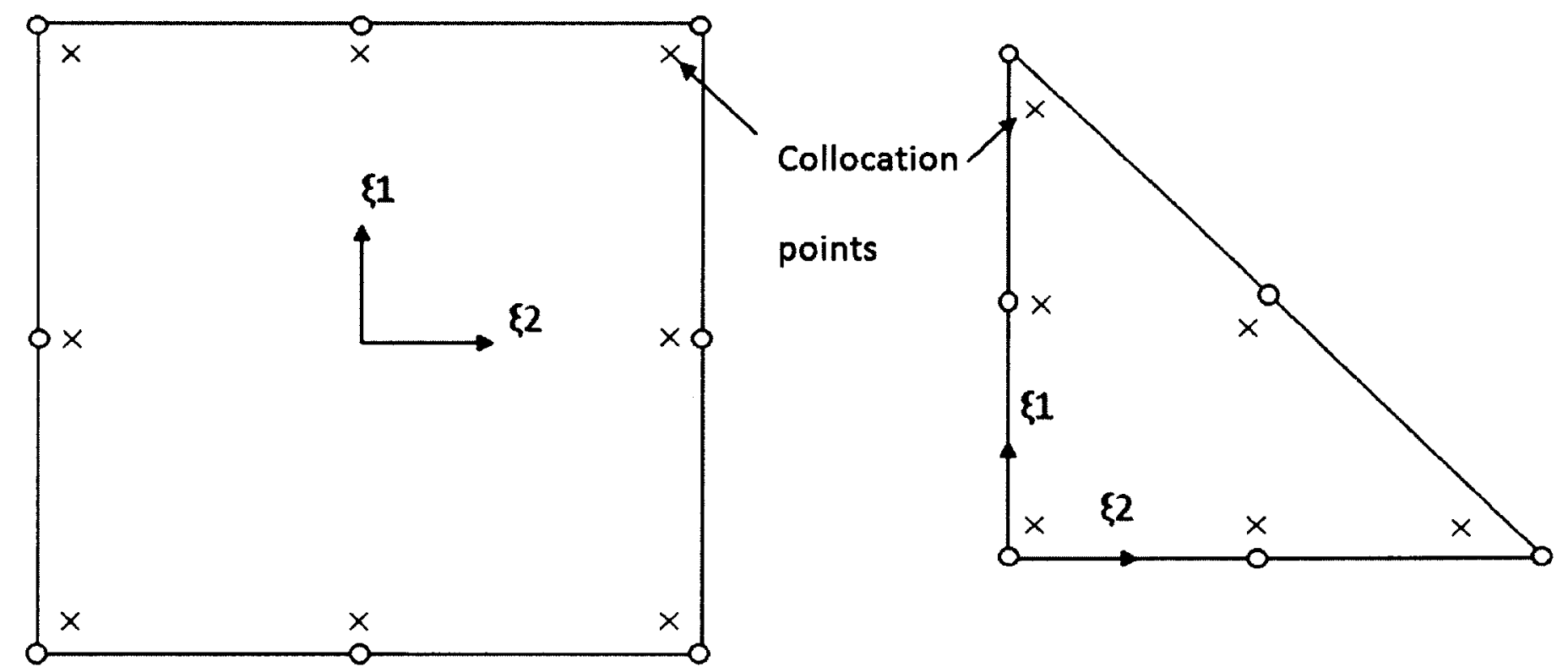

Figure 4.1: Location of the collocation points inside an element 


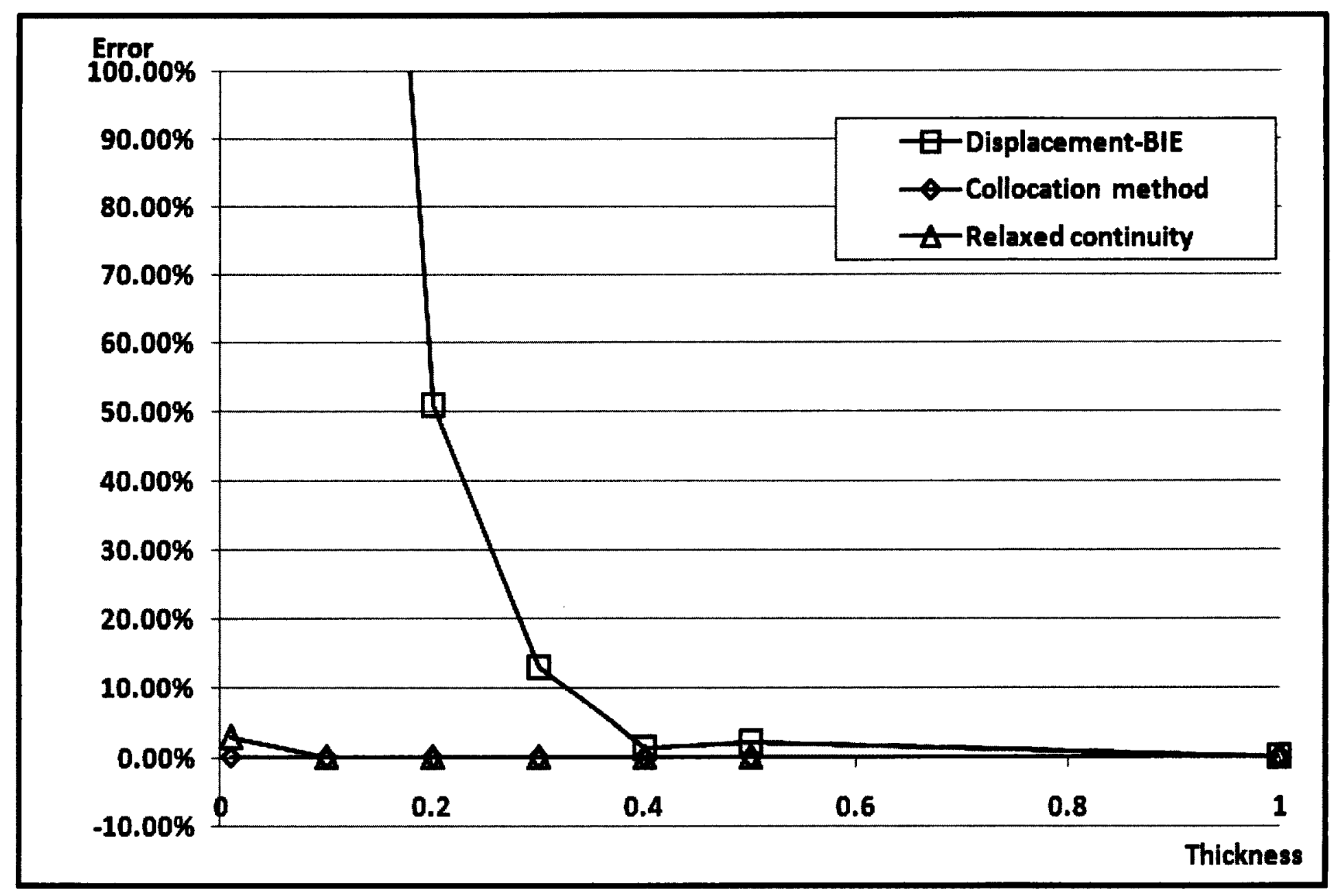

Figure 4.2: Variation of the maximum error in stresses with thickness for the thin plate problem 


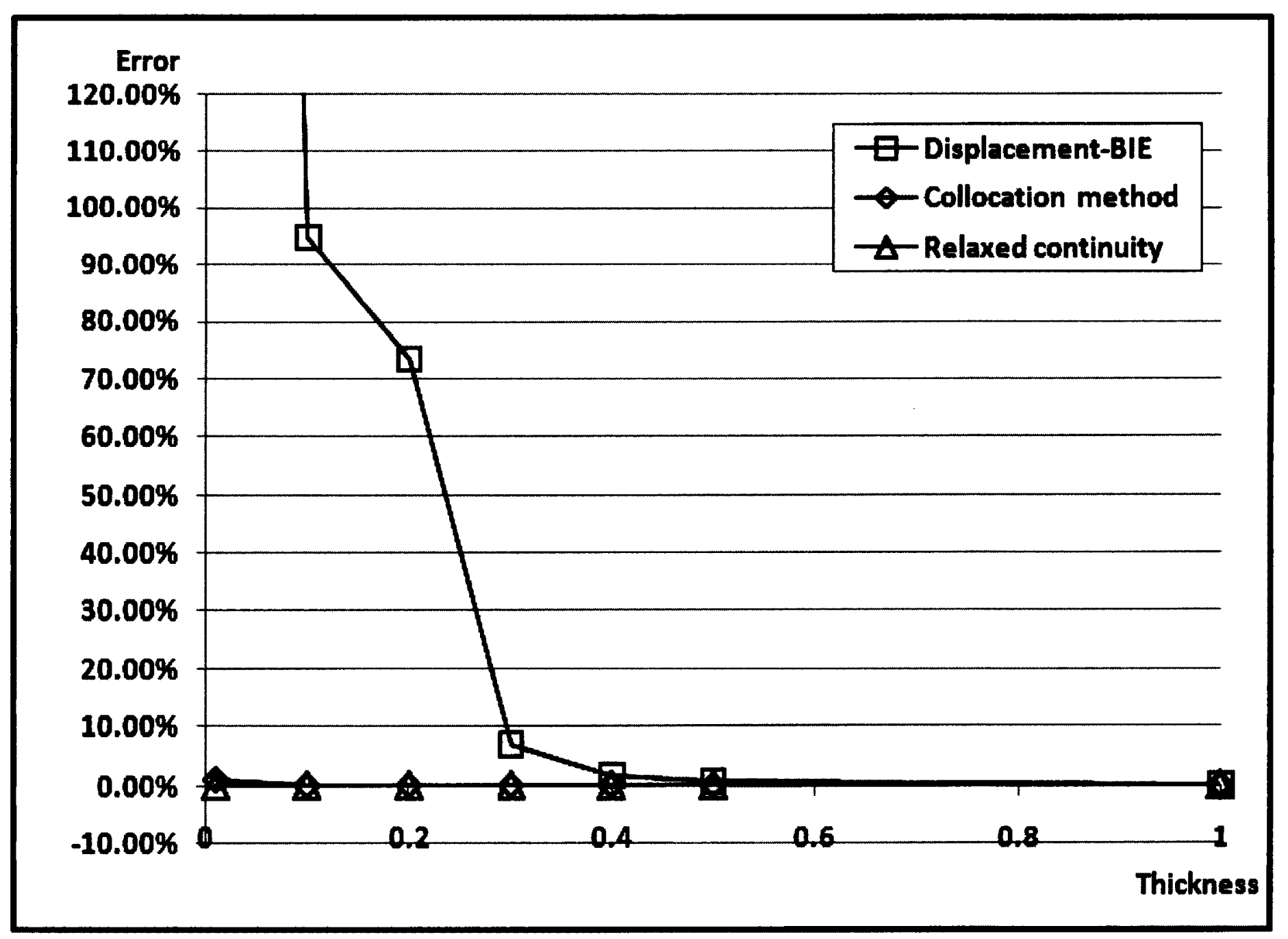

Figure 4.3: Variation of the maximum error in displacements with thickness for the thin plate problem 


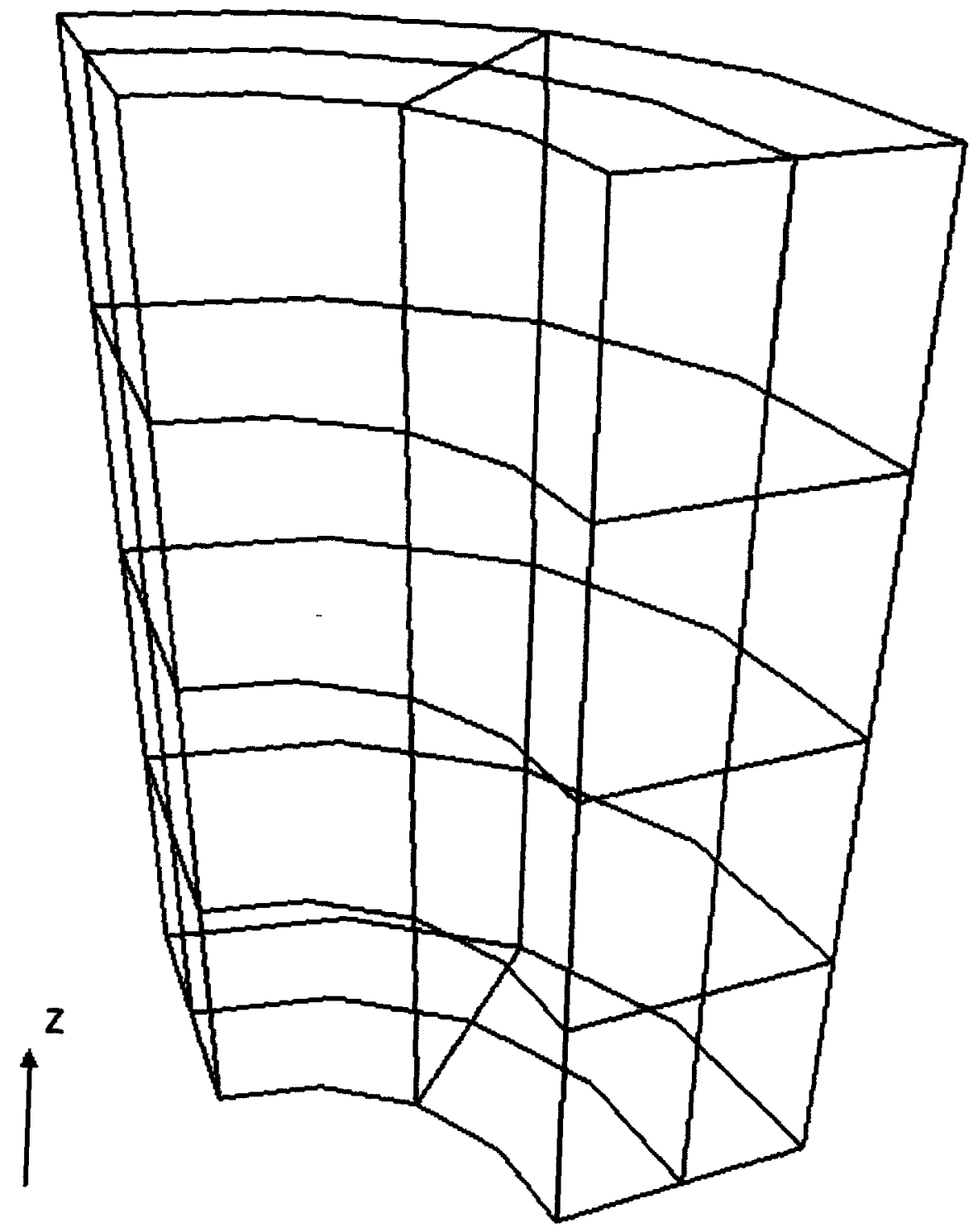

Figure 4.4: $B E M$ mesh of the $1 / 4$ cylinder $(K=2)$ 


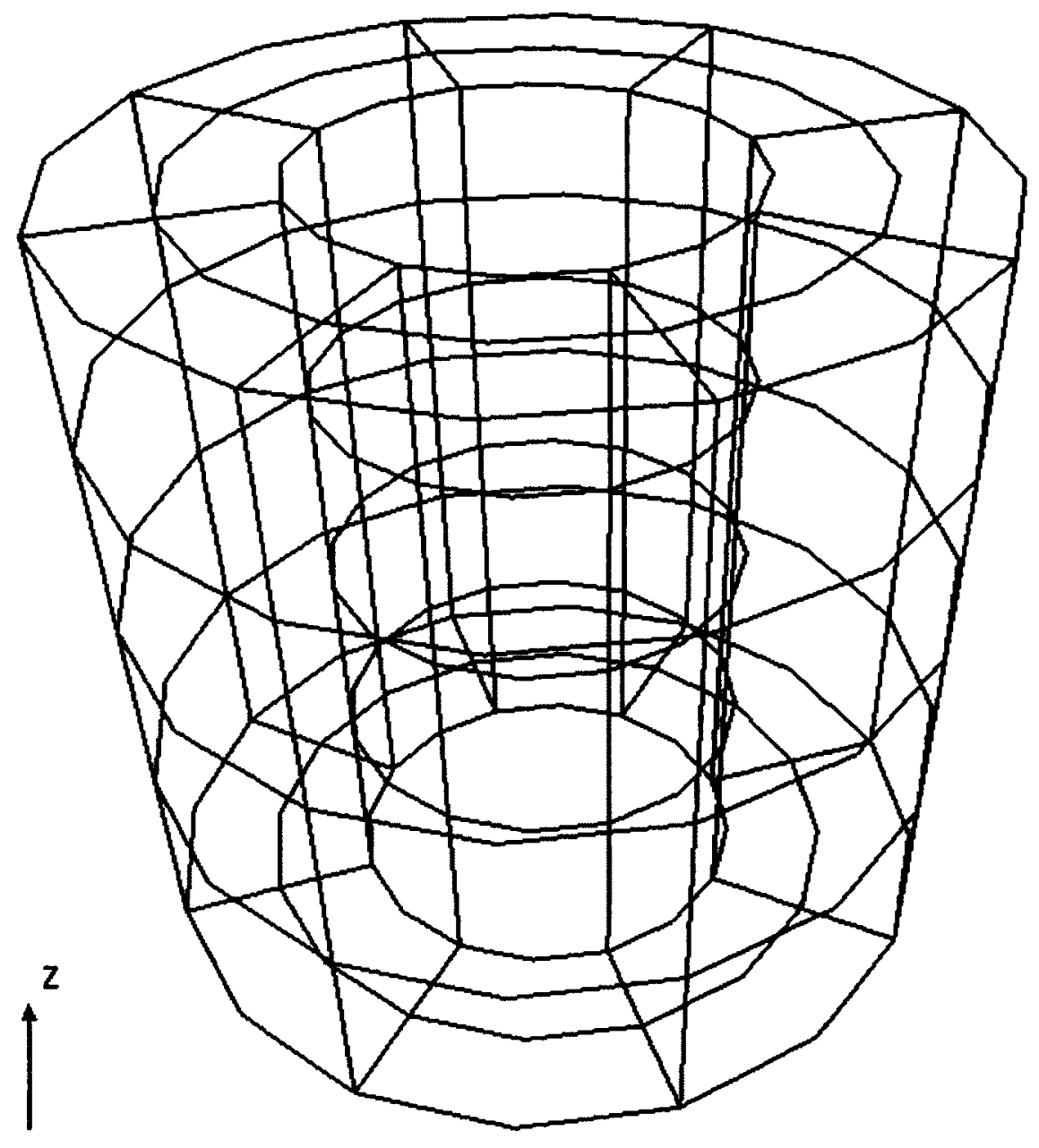

Figure 4.5: $B E M$ mesh of the fully-modeled cylinder $(K=2)$ 


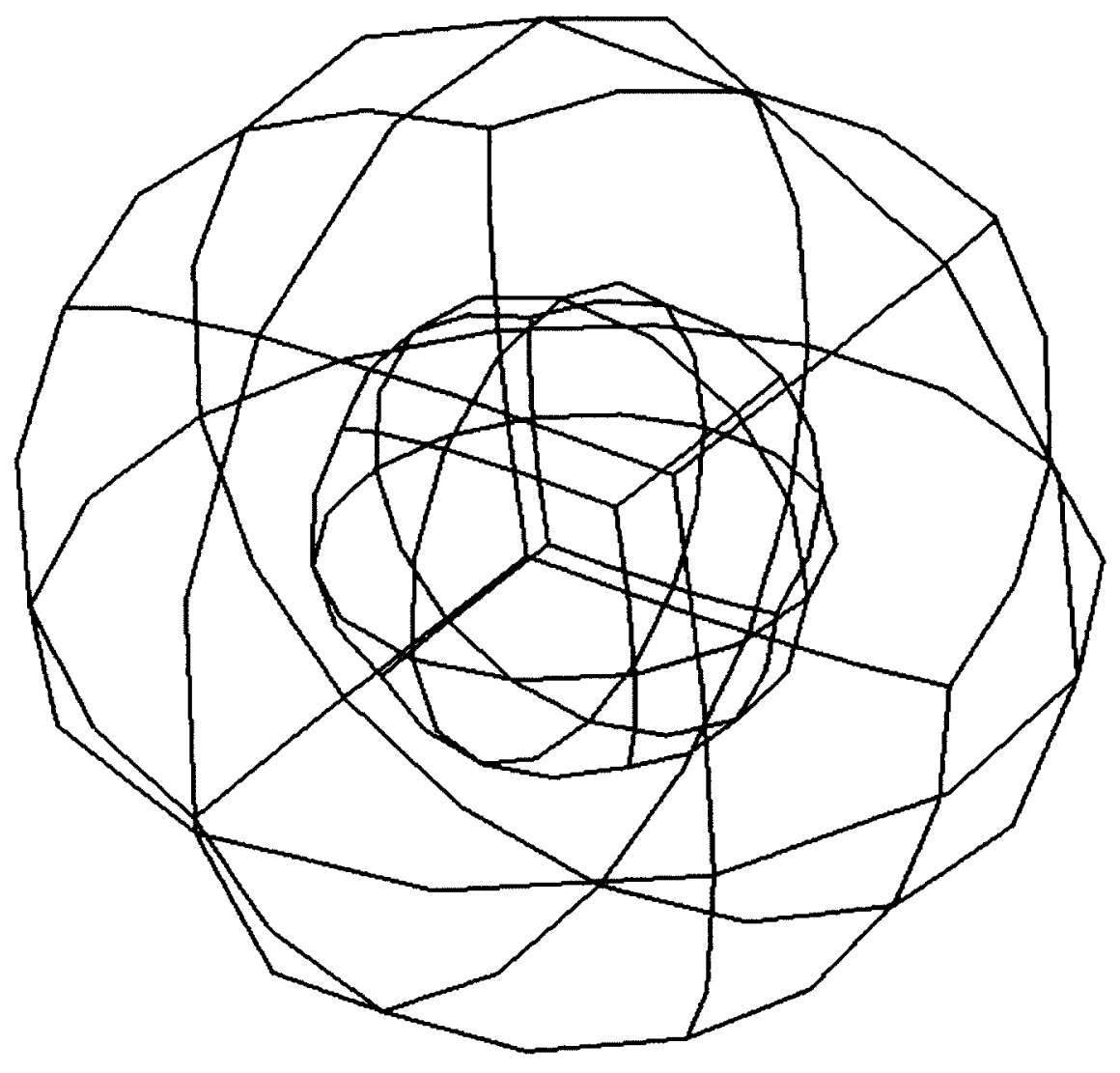

Figure 4.6: BEM mesh of the fully-modeled sphere $(K=2)$ 


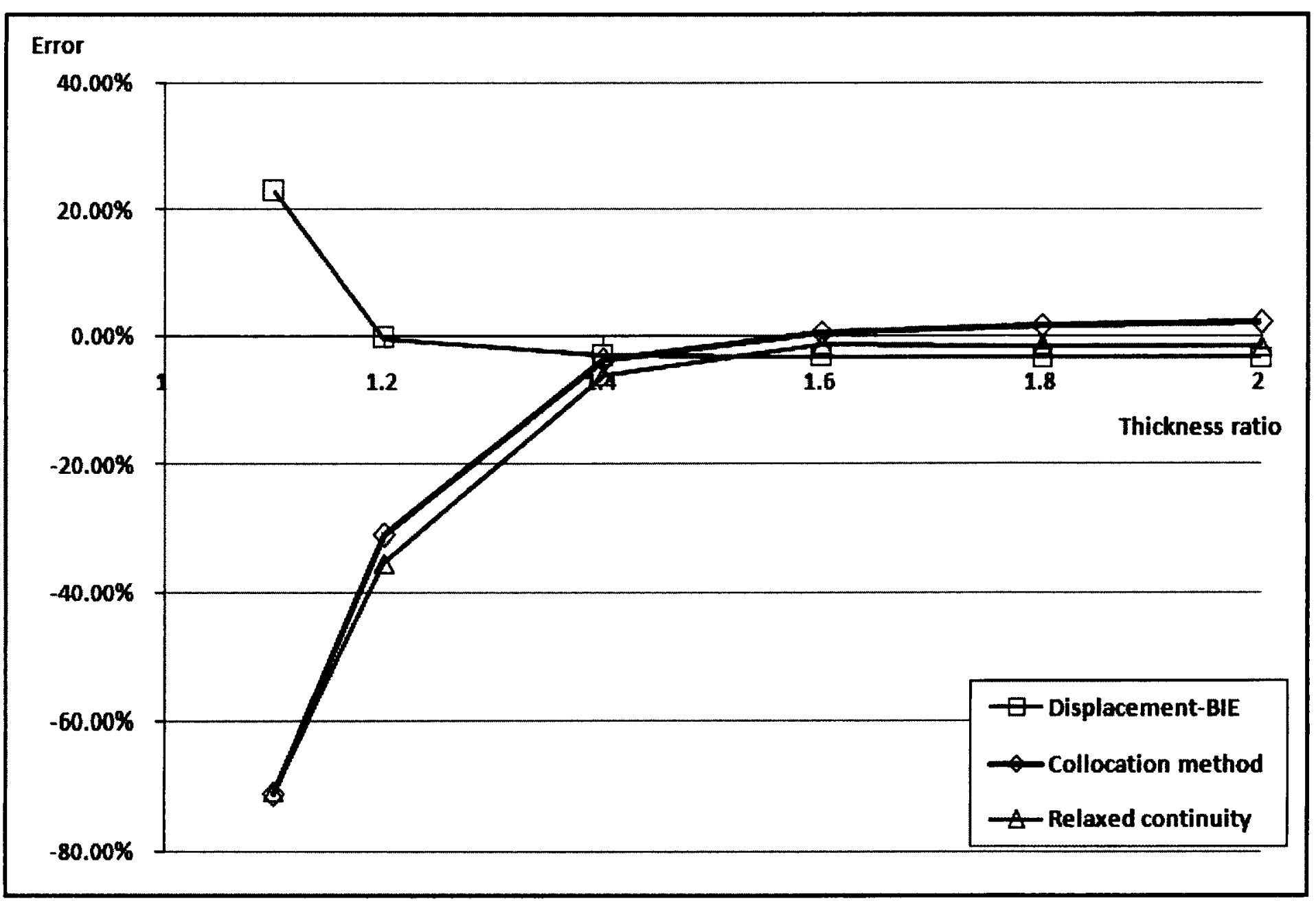

Figure 4.7: Variation of maximum error in stresses with radius ratio of the hollow sphere 


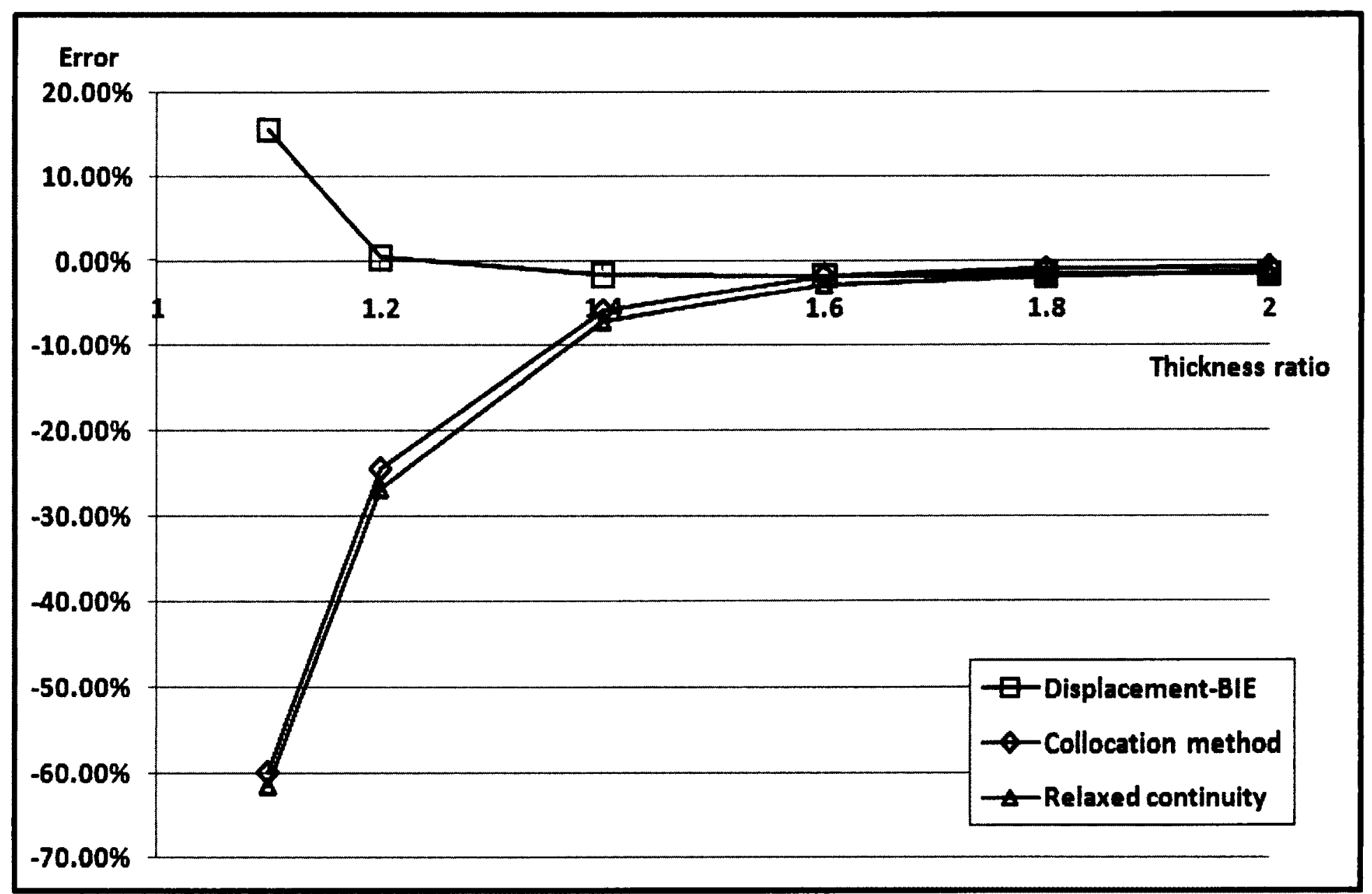

Figure 4.8: Variation of maximum error in displacements with radius ratio of the hollow sphere 


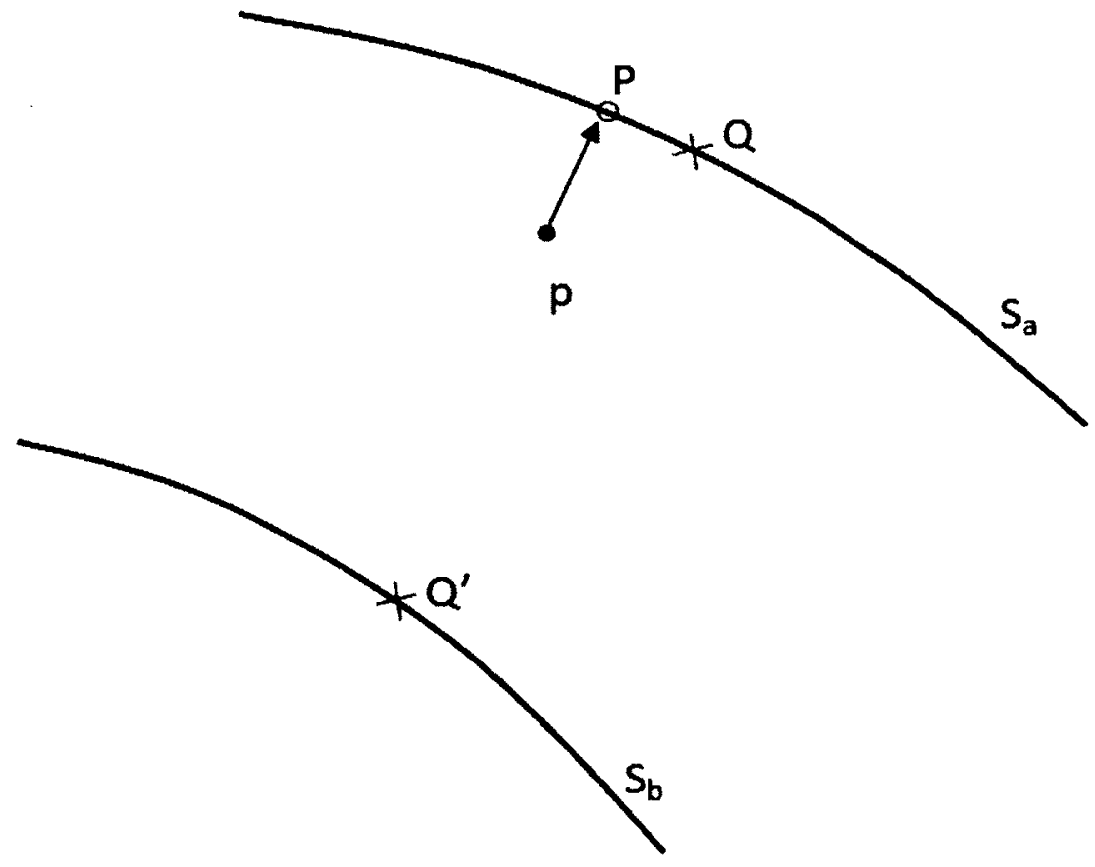

Figure 4.9: Schematic of the interior point between two surfaces 


\begin{tabular}{|c|c|c|c|c|c|c|}
\hline \multicolumn{7}{|c|}{ Relaxed continuity } \\
\hline Location & \multicolumn{3}{|c|}{ Outer surface } & \multicolumn{3}{c|}{ Inner surface } \\
\hline $\mathrm{z}$ & $\sigma_{1}$ & $\sigma_{2}$ & $\sigma_{3}$ & $\sigma_{1}$ & $\sigma_{2}$ & $\sigma_{3}$ \\
\hline 0 & 1.003 & $-6.5 \mathrm{E}-04$ & $-6.5 \mathrm{E}-04$ & 1.010 & $-1.4 \mathrm{E}-03$ & $-1.4 \mathrm{E}-03$ \\
\hline 0.125 & 1.016 & $4.4 \mathrm{E}-03$ & $4.4 \mathrm{E}-03$ & 1.003 & $-1.1 \mathrm{E}-03$ & $-1.1 \mathrm{E}-03$ \\
\hline 0.25 & 1.000 & $-3.1 \mathrm{E}-03$ & $-3.1 \mathrm{E}-03$ & 1.004 & $-1.9 \mathrm{E}-03$ & $-1.9 \mathrm{E}-03$ \\
\hline 0.375 & 0.998 & $-3.2 \mathrm{E}-03$ & $-3.2 \mathrm{E}-03$ & 1.004 & $-9.6 \mathrm{E}-04$ & $-9.6 \mathrm{E}-04$ \\
\hline 0.5 & 0.999 & $-8.0 \mathrm{E}-04$ & $-8.0 \mathrm{E}-04$ & 0.981 & $-1.2 \mathrm{E}-02$ & $-1.2 \mathrm{E}-02$ \\
\hline 0.625 & 0.999 & $-8.1 \mathrm{E}-04$ & $-8.1 \mathrm{E}-04$ & 0.996 & $3.5 \mathrm{E}-03$ & $3.5 \mathrm{E}-03$ \\
\hline 0.75 & 1.000 & $-4.4 \mathrm{E}-04$ & $-4.4 \mathrm{E}-04$ & 1.009 & $1.1 \mathrm{E}-02$ & $1.1 \mathrm{E}-02$ \\
\hline 0.875 & 1.000 & $-1.9 \mathrm{E}-03$ & $-1.9 \mathrm{E}-03$ & 1.009 & $1.2 \mathrm{E}-02$ & $1.2 \mathrm{E}-02$ \\
\hline 1 & 1.002 & $-1.9 \mathrm{E}-03$ & $-1.9 \mathrm{E}-03$ & 1.009 & $1.3 \mathrm{E}-02$ & $1.3 \mathrm{E}-02$ \\
\hline Exact & 1.00 & 0.00 & 0.00 & 1.00 & 0.00 & 0.00 \\
\hline \hline \multicolumn{6}{|c|}{ Collocation strategy } \\
\hline Location & \multicolumn{7}{|c|}{ Outer surface } & & Inner surface \\
\hline $\mathrm{z}$ & $\sigma_{1}$ & $\sigma_{2}$ & $\sigma_{3}$ & $\sigma_{1}$ & $\sigma_{2}$ & $\sigma_{3}$ \\
\hline 0 & 1.000 & $-2.4 \mathrm{E}-05$ & $-2.4 \mathrm{E}-05$ & 1.000 & $7.8 \mathrm{E}-05$ & $7.8 \mathrm{E}-05$ \\
\hline 0.125 & 1.000 & $-1.8 \mathrm{E}-05$ & $-1.8 \mathrm{E}-05$ & 1.000 & $-1.2 \mathrm{E}-05$ & $-1.2 \mathrm{E}-05$ \\
\hline 0.25 & 1.000 & $-1.2 \mathrm{E}-05$ & $-1.2 \mathrm{E}-05$ & 1.000 & $1.8 \mathrm{E}-06$ & $1.7 \mathrm{E}-06$ \\
\hline 0.375 & 1.000 & $-1.0 \mathrm{E}-05$ & $-1.0 \mathrm{E}-05$ & 1.000 & $6.0 \mathrm{E}-07$ & $5.1 \mathrm{E}-07$ \\
\hline 0.5 & 1.000 & $-1.3 \mathrm{E}-05$ & $-1.3 \mathrm{E}-05$ & 1.000 & $3.7 \mathrm{E}-07$ & $2.8 \mathrm{E}-07$ \\
\hline 0.625 & 1.000 & $-1.5 \mathrm{E}-05$ & $-1.5 \mathrm{E}-05$ & 1.000 & $-2.5 \mathrm{E}-06$ & $-2.6 \mathrm{E}-06$ \\
\hline 0.75 & 1.000 & $-7.7 \mathrm{E}-06$ & $-7.8 \mathrm{E}-06$ & 1.000 & $1.2 \mathrm{E}-07$ & $3.0 \mathrm{E}-08$ \\
\hline 0.875 & 1.000 & $6.8 \mathrm{E}-07$ & $5.9 \mathrm{E}-07$ & 1.000 & $-3.7 \mathrm{E}-07$ & $-4.6 \mathrm{E}-07$ \\
\hline 1 & 1.000 & $-2.7 \mathrm{E}-05$ & $-2.7 \mathrm{E}-05$ & 1.000 & $1.3 \mathrm{E}-05$ & $1.3 \mathrm{E}-05$ \\
\hline Exact & 1.00 & 0.00 & 0.00 & 1.00 & 0.00 & 0.00 \\
\hline & & & & \\
\hline & & & & \\
\hline
\end{tabular}

Table 4.1: Stress results for the thick-walled cylinder under axial load 


\begin{tabular}{|c|c|c|c|c|c|c|c|}
\hline & Location & \multicolumn{3}{|c|}{ Displacement-BIE } & \multicolumn{3}{c|}{ Collocation method } \\
\hline & $\mathrm{z}$ & $\sigma_{1}$ & $\sigma_{2}$ & $\sigma_{3}$ & $\sigma_{1}$ & $\sigma_{2}$ & $\sigma_{3}$ \\
\hline & 0 & 0.6745 & 0.1960 & 0.0372 & 0.7237 & 0.1913 & -0.0186 \\
\cline { 2 - 8 } & 0.125 & 0.6640 & 0.1993 & 0.0001 & 0.7587 & 0.2175 & 0.0001 \\
\cline { 2 - 8 } & 0.25 & 0.6639 & 0.1984 & 0.0001 & 0.7547 & 0.2295 & 0.0001 \\
\cline { 2 - 8 } & 0.375 & 0.6640 & 0.1992 & 0.0001 & 0.7732 & 0.2422 & 0.0001 \\
\cline { 2 - 8 } & 0.5 & 0.6639 & 0.1991 & 0.0001 & 0.7624 & 0.2444 & 0.0001 \\
\cline { 2 - 8 } & 0.625 & 0.6640 & 0.1992 & 0.0001 & 0.7732 & 0.2422 & 0.0001 \\
\cline { 2 - 8 } & 0.75 & 0.6639 & 0.1984 & 0.0001 & 0.7547 & 0.2295 & 0.0001 \\
\cline { 2 - 8 } & 0.875 & 0.6640 & 0.1993 & 0.0001 & 0.7587 & 0.2175 & 0.0001 \\
\cline { 2 - 8 } & 1 & 0.6745 & 0.1960 & 0.0372 & 0.7237 & 0.1913 & -0.0185 \\
\cline { 2 - 8 } & Exact & 0.6667 & 0.2000 & 0.0000 & 0.6667 & 0.2000 & 0.0000 \\
\hline & 0 & 1.6851 & 0.1890 & -0.9197 & 1.7846 & 0.3325 & -0.8697 \\
\cline { 2 - 8 } & 0.125 & 1.6639 & 0.1991 & -0.9995 & 1.9903 & 0.3106 & -0.9994 \\
\cline { 2 - 8 } & 0.25 & 1.6640 & 0.2011 & -0.9995 & 1.8673 & 0.2443 & -0.9994 \\
\cline { 2 - 7 } & 0.375 & 1.6633 & 0.1994 & -0.9995 & 2.0797 & 0.3108 & -0.9994 \\
\cline { 2 - 7 } & 0.5 & 1.6632 & 0.1991 & -0.9995 & 1.8719 & 0.2488 & -0.9995 \\
\cline { 2 - 8 } & 0.625 & 1.6633 & 0.1994 & -0.9995 & 2.0797 & 0.3108 & -0.9994 \\
\hline & 0.75 & 1.6640 & 0.2011 & -0.9995 & 1.8672 & 0.2443 & -0.9995 \\
\hline & 0.875 & 1.6639 & 0.1991 & -0.9995 & 1.9902 & 0.3105 & -0.9994 \\
\hline 1 & 1.6851 & 0.1890 & -0.9197 & 1.7846 & 0.3326 & -0.6264 \\
\hline & Exact & 1.6667 & 0.2000 & -1.0000 & 1.6667 & 0.2000 & -1.0000 \\
\hline
\end{tabular}

Table 4.2: Stress results for the 1/4-model of the thick-walled cylinder under internal pressure 


\begin{tabular}{|c|c|c|c|c|c|c|c|}
\hline & Location & \multicolumn{3}{|c|}{ Displacement-BIE } & \multicolumn{3}{c|}{ Collocation method } \\
\hline & $\mathrm{z}$ & $\sigma_{1}$ & $\sigma_{2}$ & $\sigma_{3}$ & $\sigma_{1}$ & $\sigma_{2}$ & $\sigma_{3}$ \\
\hline & 0 & 0.6744 & 0.1933 & 0.0367 & 0.6573 & 0.1904 & 0.0267 \\
\cline { 2 - 8 } & 0.125 & 0.6647 & 0.1988 & 0.0001 & 0.6663 & 0.1922 & 0.0001 \\
\hline 0.25 & 0.6656 & 0.2007 & 0.0001 & 0.6870 & 0.2124 & 0.0001 \\
\cline { 2 - 8 } & 0.375 & 0.6655 & 0.2003 & 0.0001 & 0.6997 & 0.2176 & 0.0001 \\
\cline { 2 - 8 } & 0.5 & 0.6653 & 0.1997 & 0.0001 & 0.6975 & 0.2189 & 0.0001 \\
\cline { 2 - 8 } & 0.625 & 0.6654 & 0.2003 & 0.0001 & 0.6996 & 0.2176 & 0.0001 \\
\cline { 2 - 8 } & 0.75 & 0.6656 & 0.2007 & 0.0001 & 0.6869 & 0.2125 & 0.0001 \\
\cline { 2 - 8 } & 0.875 & 0.6647 & 0.1989 & 0.0001 & 0.6661 & 0.1922 & 0.0001 \\
\cline { 2 - 8 } & 1 & 0.6744 & 0.1934 & 0.0367 & 0.6570 & 0.1900 & 0.0264 \\
\cline { 2 - 8 } & Exact & 0.6667 & 0.2000 & 0.0000 & 0.6667 & 0.2000 & 0.0000 \\
\hline & 0 & 1.6886 & 0.1857 & -0.9017 & 1.7298 & 0.3583 & -0.7312 \\
\cline { 2 - 8 } & 0.125 & 1.6631 & 0.1991 & -0.9995 & 1.7260 & 0.2377 & -0.9995 \\
\cline { 2 - 8 } & 0.25 & 1.6635 & 0.2002 & -0.9995 & 1.7051 & 0.2019 & -0.9995 \\
\cline { 2 - 7 } & 0.375 & 1.6631 & 0.1992 & -0.9995 & 1.7797 & 0.2147 & -0.9994 \\
\hline & 0.5 & 1.6631 & 0.1990 & -0.9995 & 1.7180 & 0.1869 & -0.9995 \\
\cline { 2 - 8 } & 0.625 & 1.6742 & 0.2031 & -0.9995 & 1.7797 & 0.2147 & -0.9994 \\
\cline { 2 - 7 } & 0.75 & 1.6635 & 0.2001 & -0.9995 & 1.7051 & 0.2015 & -0.9995 \\
\hline & 0.875 & 1.6634 & 0.1991 & -0.9995 & 1.7251 & 0.2373 & -0.9995 \\
\hline & 1 & 1.6889 & 0.1861 & -0.9003 & 1.7295 & 0.3607 & -0.7251 \\
\cline { 2 - 7 } & Exact & 1.6667 & 0.2000 & -1.0000 & 1.6667 & 0.2000 & -1.0000 \\
\hline
\end{tabular}

Table 4.3: Stress results for the fully-modeled thick-walled cylinder under internal pressure 


\section{Chapter 5}

\section{Self-regularized Displacement-BIE for Thin-body Problems}

\subsection{Introduction}

In the previous chapter, the self-regularized traction-BIE proposed by Richardson and Cruse (1999) was shown to be not suitable for treating thin-body problems. This is due to the near singularity arising from the second near surface for which the scheme could not accommodate. In this chapter, a modified scheme similar to the self-regularized traction-BIE, but through a different approach applied to the displacement-BIE, is proposed. This new scheme is first described and a preliminary study is carried out with some simple examples to establish the veracity of the formulation and its implementation.

\subsection{Self-regularized Displacement-BIE}

As discussed in Section 4.4.4, there are two groups of integrals that need to be regularized when treating thin-body problems, i.e. those over $S_{a}$ and the others over $S_{b}$, as in Figure 4.9. The self-regularized traction-BIE in the previous chapter only 
regularized the integrals over the elements on $S_{a}$, but provides no means to regularize those on $S_{b}$. The method proposed in this chapter uses the displacement-BIE along with the sub-division of elements, as discussed in Chapter 2, to regularize integrals on $S_{a}$, and then adopts the idea of self-regularization to regularize other integrals on $S_{b}$.

Following the development in Chapter 3 , for a reference boundary point $P_{0}$ on $S_{b}$, the displacement-BIE for constant stress state expression at $P_{0}$ may be written as:

$$
C_{i j}\left(u_{j}(Q)-u_{j}^{L}\left(P_{0}, P\right)\right)+\int_{S} T_{i j}(P, Q)\left(u_{j}(Q)-u_{j}^{L}\left(P_{0}, Q\right)\right) d S(Q)=\int_{S} U_{i j}(P, Q)\left(t_{j}(Q)-t_{j}^{L}\left(P_{0}, Q\right)\right) d i
$$

where

$$
\begin{aligned}
u_{j}^{L}\left(P_{0}, P\right) & =u_{j}\left(P_{0}\right)+u_{j, m}\left[x_{m}(P)-x_{m}\left(P_{0}\right)\right] \\
u_{j}^{L}\left(P_{0}, Q\right) & =u_{j}\left(P_{0}\right)+u_{j, m}\left[x_{m}(Q)-x_{m}\left(P_{0}\right)\right] \\
t_{j}^{L}\left(P_{0}, Q\right) & =\sigma_{j m}\left(P_{0}\right) n_{m}(Q)
\end{aligned}
$$

Subtracting Equation (5.1) from Equation (2.1) yields:

$$
\begin{aligned}
C_{i j}\left[u_{j}(P)-u_{j}^{L}\left(P_{0}, P\right)\right] & +\int_{S} T_{i j}(P, Q)\left[u_{j}(Q)-u_{j}^{L}\left(P_{0}, Q\right)\right] d S(Q) \\
& =\int_{S} U_{i j}(P, Q)\left[t_{j}(Q)-t_{j}^{L}\left(P_{0}, Q\right)\right] d S(Q)
\end{aligned}
$$

Similar to the self-regularized traction-BIE, the constant stress terms $u_{j}^{L}\left(P_{0}, Q\right)$ and $t_{j}^{L}\left(P_{0}, Q\right)$ are expressed by the displacements and tractions at the nodes of the element 
containing $P_{0}$, i.e.

$$
u_{j}^{L}\left(P_{0}, P \text { or } Q\right)=\sum_{i=1}^{8 \text { or } 6} N_{i} u_{j}^{i}\left(\xi_{1}^{P_{0}}, \xi_{2}^{P_{0}}\right)+C_{k m r} t_{r}\left(\xi_{1}^{P_{0}}, \xi_{2}^{P_{0}}\right)+D_{k m r} \sum_{i=1}^{8 \text { or } 6} N_{i}^{\prime} u_{r}^{i}\left(\xi_{1}^{P_{0}}, \xi_{2}^{P_{0}}\right)
$$

and

$$
t_{j}^{L}\left(P_{0}, Q\right)=E_{k m r} t_{r}\left(\xi_{1}^{P_{0}}, \xi_{2}^{P_{0}}\right)+F_{k m r} \sum_{i=1}^{8 \text { or } 6} N_{i}^{\prime} u_{r}^{i}\left(\xi_{1}^{P_{0}}, \xi_{2}^{P_{0}}\right)
$$

Note that in the matrix $[D]$ in Equation (4.4), point $P$ is now $P_{0}$ and $Q$ is either $Q$ or $P$, while the matrix $[\mathbf{S}]$ in Equation (4.5) remains unchanged.

Rearranging the coefficients so that those corresponding to the displacements are placed into the matrix $[\mathbf{A}]$ in Equation (2.21) and those corresponding to the tractions placed into $[\mathrm{B}]$, the system of equations is now ready to be solved.

In conventional displacement-BIE, as described in Chapter 2 , the free terms $C_{i j}(P)$ are calculated indirectly by considering the rigid-body motion condition. In Equation (5.3), however, the $C_{i j}(P)$ terms appear not only at the diagonal sub-matrices of the system matrix $[\mathbf{A}]$, they are also placed in columns corresponding to $P_{0}$ with the negative sign. If the off-diagonal coefficients in each line of the matrix [A] are summed up, the $C_{i j}$ terms at these two places will cancel each other out. The use of rigid-body motion conditions is thus not appropriate for calculating the $C_{i j}$ terms here. An alternative approach of direct evaluation of the free terms is thus employed. This will be discussed in the next section.

Using the above self-regularized displacement-BIE, the integrals over the boundary elements on the surface containing $P$ and on the surface near $P$ can be regularized at the same time. No singularity issues are of concern now and this BIE should thus 
be able to solve problems involving thin bodies.

\subsection{Evaluation of the Free Terms $C_{i j}$}

As discussed above, the $C_{i j}$ terms at source point $P$ cannot be calculated indirectly by considering the rigid-body motion condition as is usually done in conventional displacement-BIE. An alternative way is to evaluate these terms analytically, although it is numerically not as simple. Such an approach has been proposed by Dangla et al. (2005), and is adopted in this study. The expression for $C_{i j}$ is given as follows:

$$
C_{i j}(P)=\frac{\psi}{4 \pi} \delta_{i j}-\frac{1}{8 \pi(1-\nu)} \sum_{e=1}^{E} \sin \left(\frac{\theta^{e}}{2}\right)\left(b_{i}^{e} n_{j}^{e}+b_{j}^{e} n_{i}^{e}\right)
$$

where $E$ is the number of elements sharing the node $P, \theta^{e}$ is the angle formed by the two edges of element $e$ at $P, b_{i}^{e}$ is the unit vector of the bisecting line of these two edges, and $n_{i}^{e}$ is the unit outward normal of element $e$. These quantities are illustrated in Figure 5.1, with a unit sphere centered at $P$.

The solid angle, $\psi$, can be calculated using

$$
\psi=\sum_{e=1}^{E}\left(\psi_{1}^{e}+\psi_{2}^{e}+\psi_{3}^{e}-\pi\right)
$$

where $\psi_{i}^{e}$ are the angles formed by the planes of the trihedron as shown in Figure 5.2. This trihedron is formed by the two edges of element $e$, the semi-axis in the direction of $-n(P)$, and the part of the unit sphere cut off by these three lines, where $n(P)$ is an arbitrarily chosen normal at $P$, usually taken as the average of outward normals of the elements sharing $P$. It was stated in Dangla et al. (2005) that the choice of this $n(P)$ does not affect the accuracy of the free terms since the solid angle 
will be recovered whatever the $n(P)$ is. Since $n(P)$ is the average of the normals of the elements sharing $P$, it will always be located between the edges of the elements. Thus, the sum of $\psi_{3}^{e}$ will become $2 \pi$, and Equation (5.7) becomes

$$
\psi=\sum_{e=1}^{E}\left(\psi_{1}^{e}+\psi_{2}^{e}\right)+2 \pi-E \pi
$$

To obtain the angles $\psi_{i}^{e}$, we need the vectors $\mathbf{v}_{\mathbf{i}}$ as shown in Figure 5.2. These vectors are obtained as follows:

$$
\begin{array}{r}
\mathbf{v}_{1}=\mathbf{n}^{\mathbf{e}} \times \mathbf{a}_{1} \\
\mathbf{v}_{\mathbf{2}}=\mathbf{a}_{1} \times\left(\mathbf{n}(\mathbf{P}) \times \mathbf{a}_{1}\right) \\
\mathbf{v}_{3}=\mathbf{a}_{2} \times \mathbf{n}^{\mathbf{e}} \\
\mathbf{v}_{4}=\mathbf{a}_{2} \times\left(\mathbf{n}(\mathbf{P}) \times \mathbf{a}_{2}\right)
\end{array}
$$

where $\mathbf{a}_{1}$ and $\mathbf{a}_{\mathbf{2}}$ are the unit vectors of the two edges. With $\mathbf{v}_{\mathbf{i}}, \psi_{i}$ can be obtained by:

$$
\begin{aligned}
& \psi_{1}=\cos ^{-1}\left(\frac{\mathbf{v}_{\mathbf{1}} \mathbf{v}_{\mathbf{2}}}{\left|\mathbf{v}_{\mathbf{1}}\right|\left|\mathbf{v}_{\mathbf{2}}\right|}\right) \\
& \psi_{2}=\cos ^{-1}\left(\frac{\mathbf{v}_{\mathbf{3}} \mathbf{v}_{\mathbf{4}}}{\left|\mathbf{v}_{\mathbf{3}}\right|\left|\mathbf{v}_{\mathbf{4}}\right|}\right)
\end{aligned}
$$

Once all the quantities in Equation (5.6) are obtained, the free terms $C_{i j}$ can be computed and appropriately placed into the matrix $[\mathbf{A}]$, corresponding to the node $P$ and nodes of the element containing $P_{0}$.

The algorithm for locating the regularization point in Chapter 3 has been 
modified so that it searches for a surface point $P_{0}$ whose outward normal is parallel to the vector from source point $P$ to $P_{0}$. Note that $P_{0}$ and $P$ do not belong to the same boundary element. This can ensure the regularization point is located on the second near surface containing near singularities, when dealing with thin-body problems. Using the self-regularization technique, the displacement-BIE in Equation (5.3) can be used for treating such problems without the need of very significant mesh refinement as in conventional BEM analysis.

\subsection{Numerical Examples}

Some numerical examples involving thin bodies are presented here to support the selfregularized displacement-BIE proposed in this chapter. These examples are similar to those used in the previous chapters but are made thinner across the solid crosssections.

\subsubsection{Thin Plate under Uniform Stresses}

The simple thin plate problem in Chapter 4 is tested again here; Figure 5.3 shows the BEM mesh of the problem, with one element on each surface. The material properties and loadings are the same as before. The thickness of the $1 \times 1$ plate is gradually reduced from 1 to 0.0001 . The maximum errors in stresses and displacements are plotted with variation of the thickness in Figure 5.4. As can be seen in the figure, the errors are very low until the thickness reaches 0.0001 ; this is in contrast to the results from using the conventional displacement-BIE seen in the previous chapter, where the errors increase significantly when the relative thickness is decreased below 0.3 using the same mesh. The deterioration at $t=0.0001$ was established to be caused by the large aspect ratio of the elements across the thickness, rather than the algorithm itself. This issue will be further discussed later. 


\subsubsection{Thin-walled Cylinder and Sphere}

The internally pressurized cylinder and sphere problems that the self-regularized traction-BIE failed in Chapter 4 are tested again, but only in the category of thin-walled vessels. The Young's modulus is set to 1000 units and Poisson's ratio is 0.3. The requirement for $C^{1, \alpha}$-continuity in the traction-BIE formulation is no longer an issue here. Thus, a quarter-model for the cylinder and an one-eighth model for the sphere are used instead of full models. The regularization points of the nodes on the inner/outer surface are located on the opposing surface to regularize the singular kernels.

The initial mesh for the thin-walled cylinder is shown in Figure 5.5; it has 28 elements and 86 nodes. The inner radius of the cylinder remains at 1 while the outer radius is reduced from 1.1 to 1.001 , so that the thickness $t$ decreases from 0.1 to 0.001 . The results at a series of nodes on the inner surface, located from the bottom $(z / H=0)$ to the top surface $(z / H=1)$, are examined. The errors in stresses are shown in Figures 5.6, 5.7 and 5.8. It can be seen that the errors at the nodes on the smooth surface are within $2 \%$ until $t=0.001$. The degeneration of the accuracy at $t=0.001$ is possibly due to the relatively coarse mesh, thus a refined mesh is then tested for this case, as shown in Figure 5.9. The refined mesh has 160 elements and 482 nodes. The variation of errors along a vertical line on the inner surface is shown in Figure 5.10. The errors at smooth surface have now been reduced to within $2 \%$ again, which demonstrates that with an appropriate mesh refinement, the method can still give very good results.

In all the results presented above, at the corner nodes, i.e., where vertical location is 0 or 1 , the radial stresses have very high errors. The possible reason for 
this is, again, the very large aspect ratio (up to 1000) of the elements presenting the wall thickness. This problem is actually experienced in FEM as well, where elements with large aspect ratios are not considered to be desirable. To verify that this is indeed the source of error, a quick investigation is conducted where the mesh is refined progressively by dividing the length of the cylinder and the quarter arcs into more elements, for both BEM and FEM. 20-node brick elements are used in the FEM analysis for direct equivalent comparison. Figure 5.11 shows the variation of radial stresses at the corner adjacent to inner surface obtained from BEM solution, as well as those obtained at an arbitrary node from the FEM solution. As the aspect ratio decreases, the errors in the radial stress decrease in both BEM and FEM, but with a very slow convergency; a very refined mesh will be needed to recover the correct radial stress. Due to this reason, no more tests were conducted below the aspect ratio of 6.66667 which gives the radial stresses of error of $20 \%$, but a partially-refined mesh, as shown in Figure 5.12, is tested for $t=0.1$. The mesh is only refined around the corners so that the aspect ratio has been reduced to around 1. Using this mesh, the errors of radial stresses at the corners are found to be within $3 \%$. This shows that by eliminating the large aspect ratio, the approach can give accurate results everywhere on the surface. Notwithstanding the percentage errors in the radial stress discussed above, it should be emphasized that for the thin-walled cylinder, the hoop stress is at least an order of magnitude larger that the radial stress.

It should be noted that for the FEM analysis, all the nodes on the inner/outer surface have the same principal stresses since the interior of the body is meshed by elements having surfaces of large aspect ratio. In the BEM, on the other hand, since the interior of the body remains continuous, the error only occurs at corners with elements having large aspect ratios, while the nodes on the smooth surface still give very accurate radial stresses. This shows that when using BEM for a particular 
problem, mesh refinement might be only needed for elements with relatively large aspect ratios. In FEM, however, every element having surfaces with large aspect ratio needs to be refined as the elements are three-dimensional. This would significantly increase the number of elements and nodes, as well as computing resources required.

The mesh of the $\frac{1}{8}$-th model for the pressurized sphere is shown in Figure 5.13. It has 36 elements and 110 nodes. The inner radius is kept at unity, and the thickness $t$ is reduced from 0.1 to 0.001 so that the thickness ratio $K$ decreases from 1.1 to 1.001 . As discussed above, the stresses at the corners have relatively high errors due to the large aspect ratio, thus only those on the smooth surface are investigated. Figure 5.14 shows the maximum error of the circumference stresses at an arbitrary node on the inner surface with the variation of thickness ratio $K$. The regularized displacement-BIE gives less than $3 \%$ errors for thickness up to 0.005 and starts to deteriorate at $t=0.001$. This is, again, due to the large aspect ratio of the elements across the wall thickness. Note that in Chapter 4 , the conventional displacement-BIE starts to fail at $t=0.1$ and the self-regularized traction-BIE fails at $t=0.4$. This example demonstrates again the accuracy and efficiency of the proposed self-regularized displacement-BIE when dealing with thin-body problems, as long as due care is taken to avoid elements with large aspect ratios at the points of interest.

\subsection{Concluding Remarks}

In this chapter, a self-regularized displacement-BIE for thin-body problems has been proposed similar to the traction-BIE, but through a different approach. The $C_{i j}$ terms have to be computed analytically rather than using rigid body motion considerations as in conventional BEM analysis. The numerical examples have 
demonstrated that the thickness of a thin-walled vessel can be reduced to a very small value, if the mesh is refined so that the aspect ratio of elements across thickness remains in a reasonable range.

The proposed self-regularized displacement-BIE is relatively simple to implement, unlike most of the regularization algorithms that have hitherto been employed in BEM. The preliminary tests carried out and presented in this chapter show very promising results indeed for this formulation to be used in the elastic stress analysis of thin bodies in BEM. Clearly, a more extensive range of tests involving complex boundary conditions and geometries need to be conducted to finally establish the veracity of this scheme. The issue of having an appropriate aspect ratio of the elements at corners of the numerical solution domain also needs to be further investigated for perhaps a better remedy than more mesh refinement. The key advantage of the proposed scheme is that the same numerical algorithm and type of elements ( $C^{0}$-continuous) can be employed to treat physical problems with cross-sections varying from relatively thick to very thin, such as aircraft turbine blades with the root attachments. 


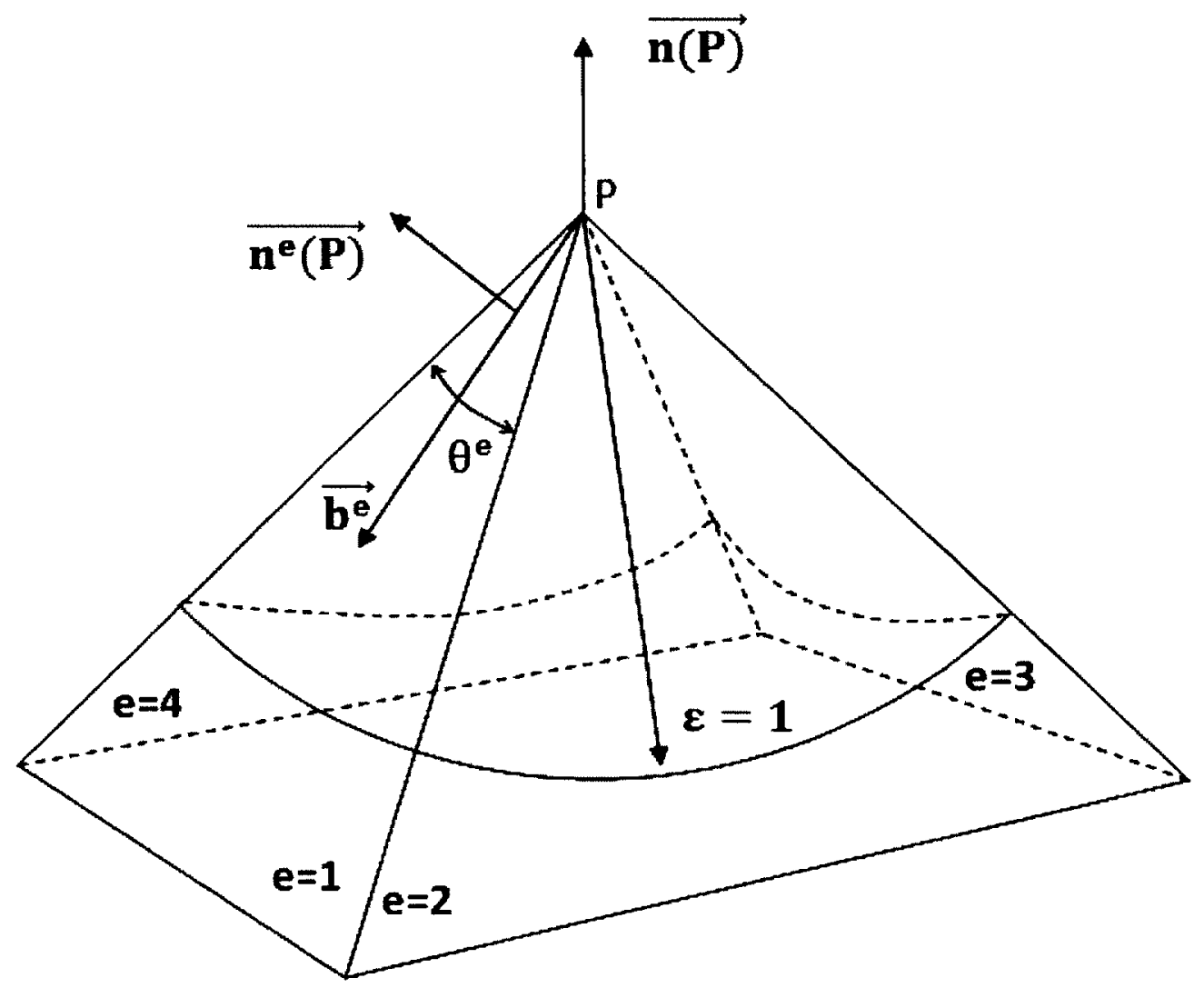

Figure 5.1: Schematic of elements sharing source point $P$ 


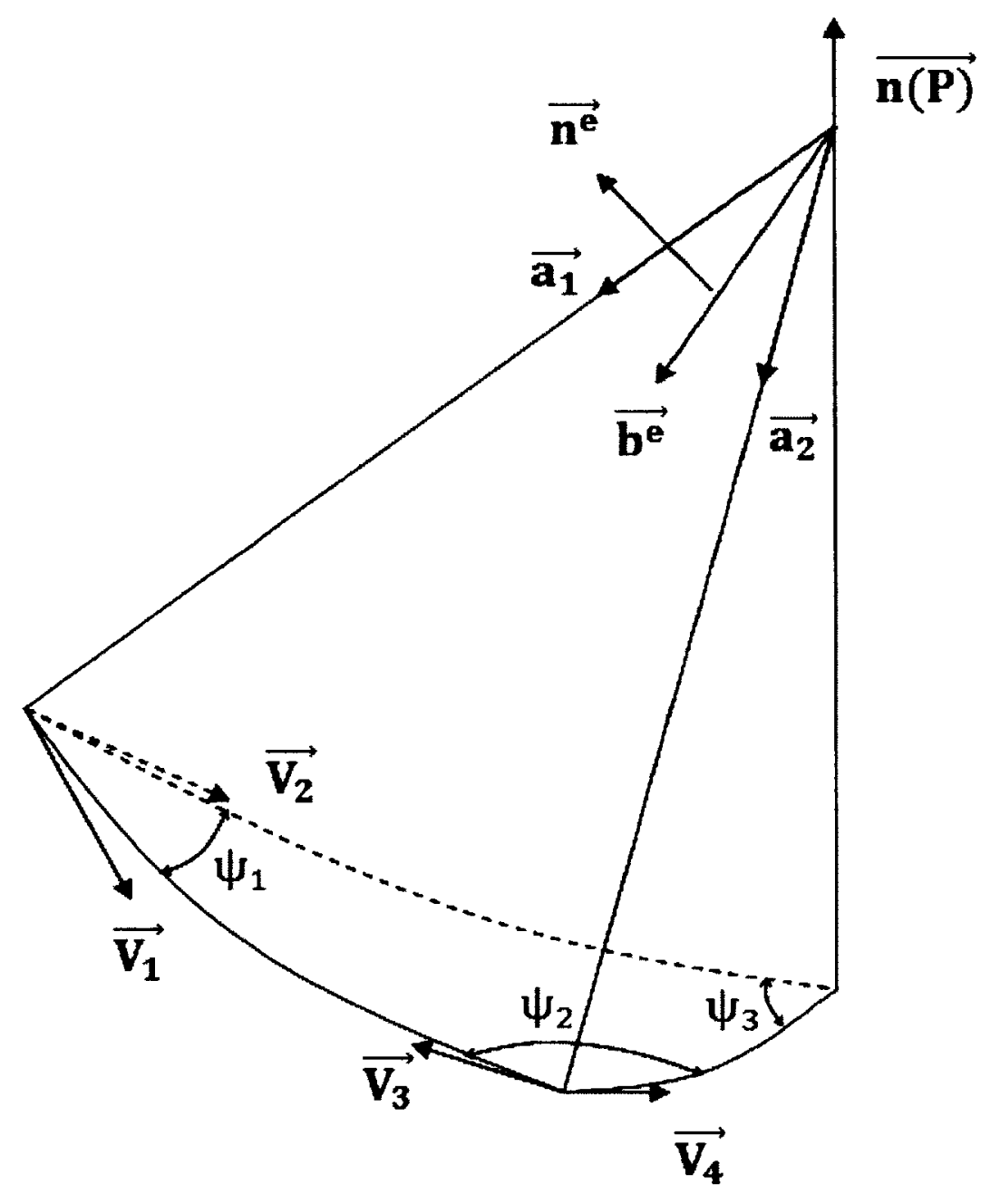

Figure 5.2: Illustrative figure for solid angle $\psi$ 


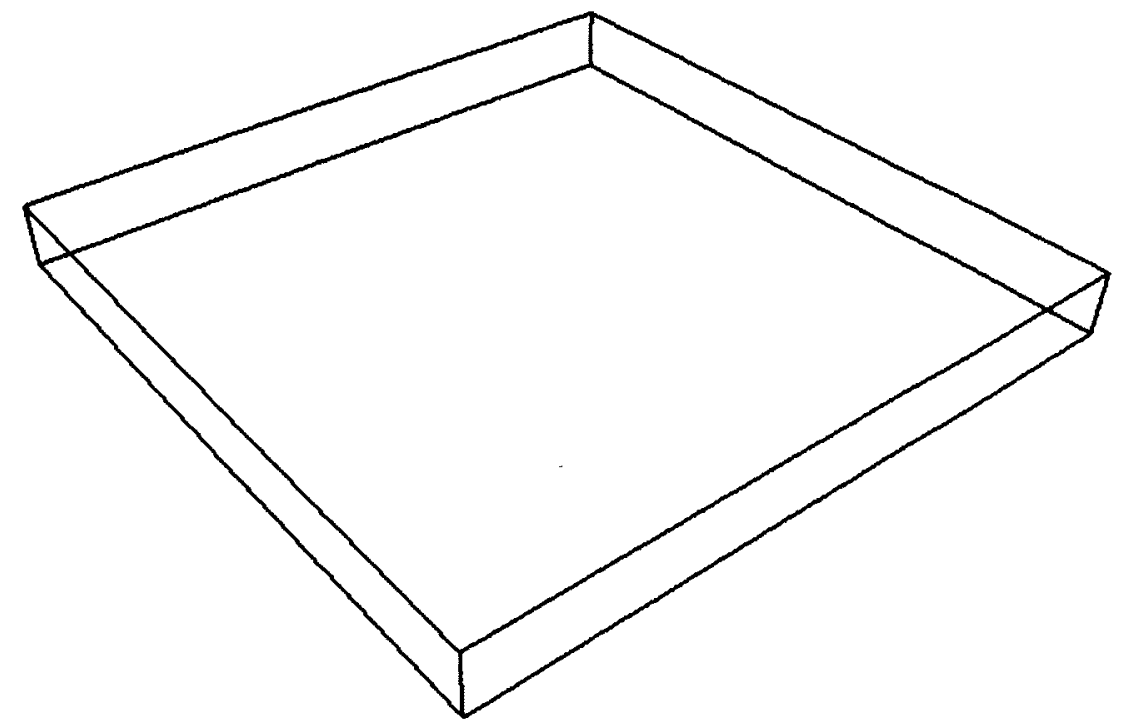

Figure 5.3: Boundary element mesh of the thin plate problem 


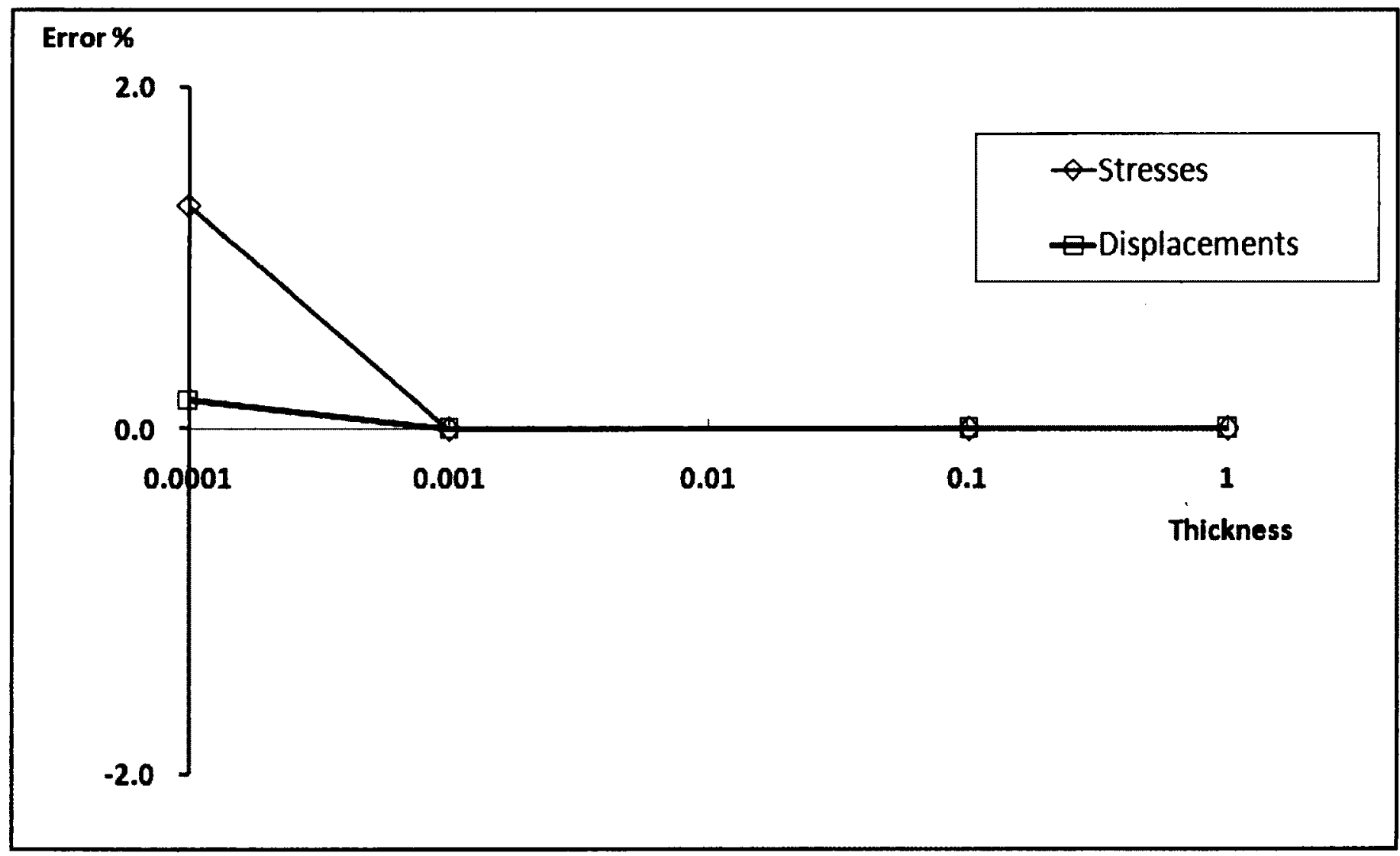

Figure 5.4: Variation of the percentage errors of the numerical results for the thin-plate problem with thickness 


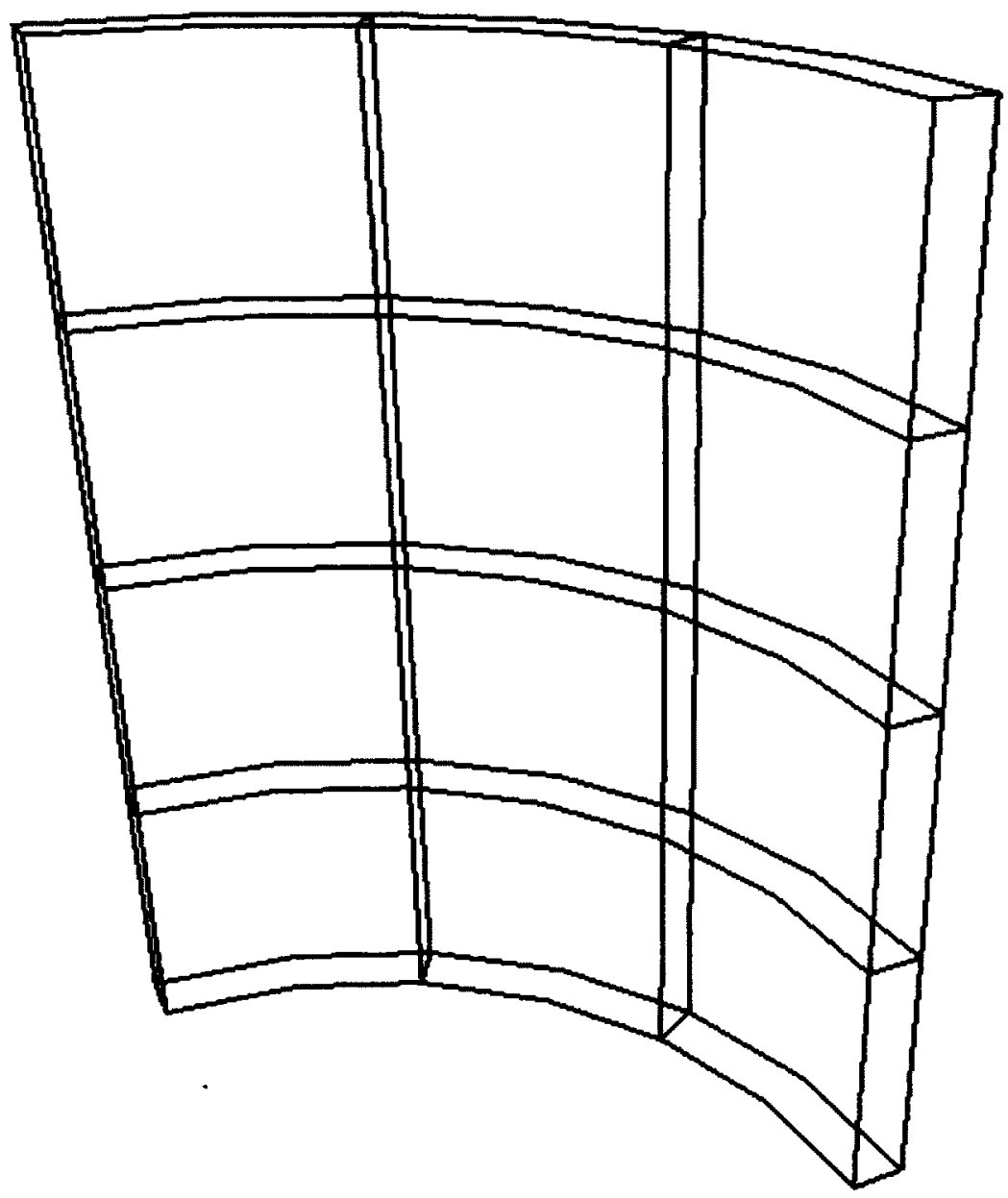

Figure 5.5: Initial boundary element mesh of the thin-walled cylinder 


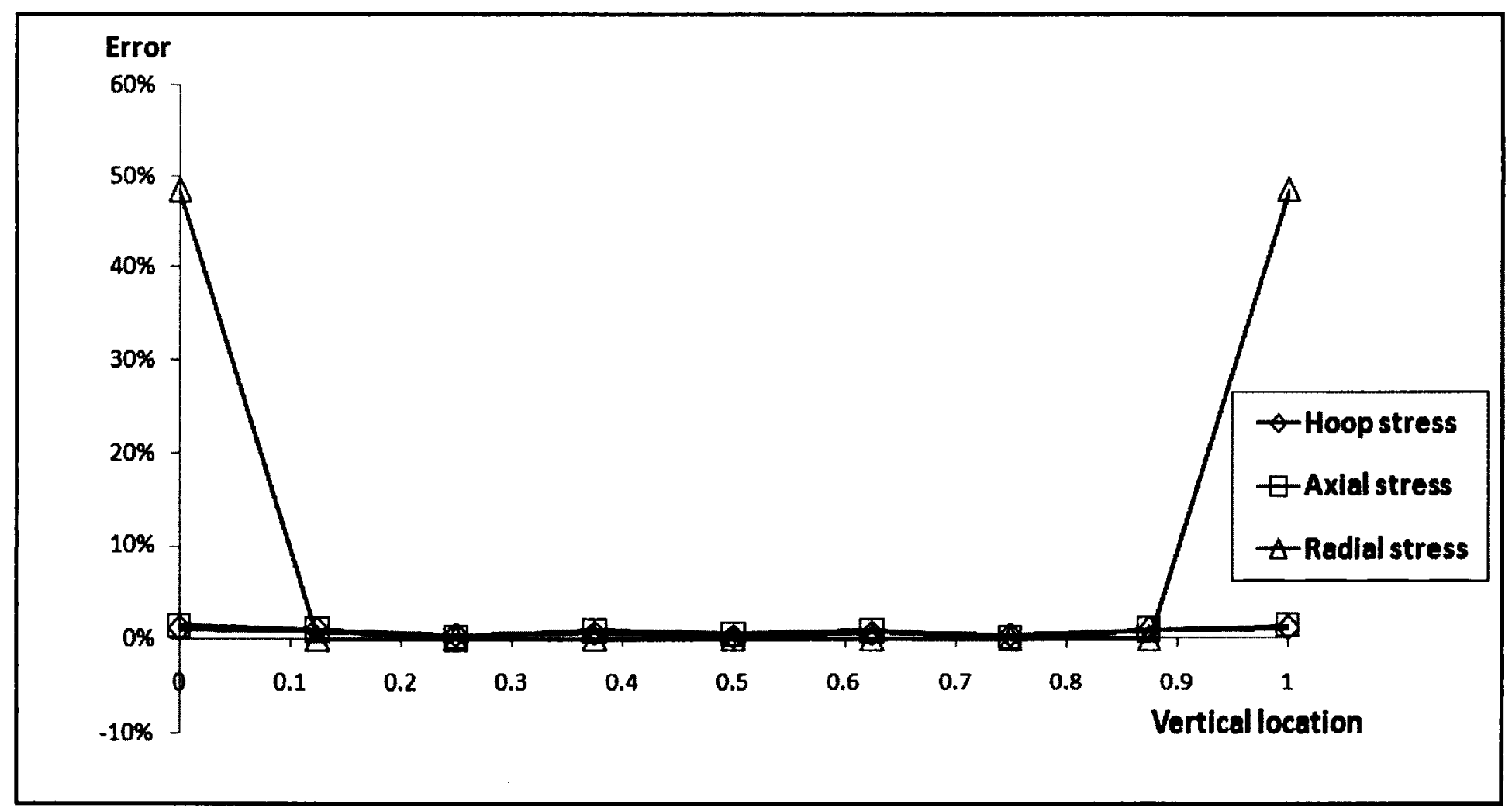

Figure 5.6: Percentage errors in the stresses of the pressurized thin-walled cylinder problem $(t=0.1)$ 


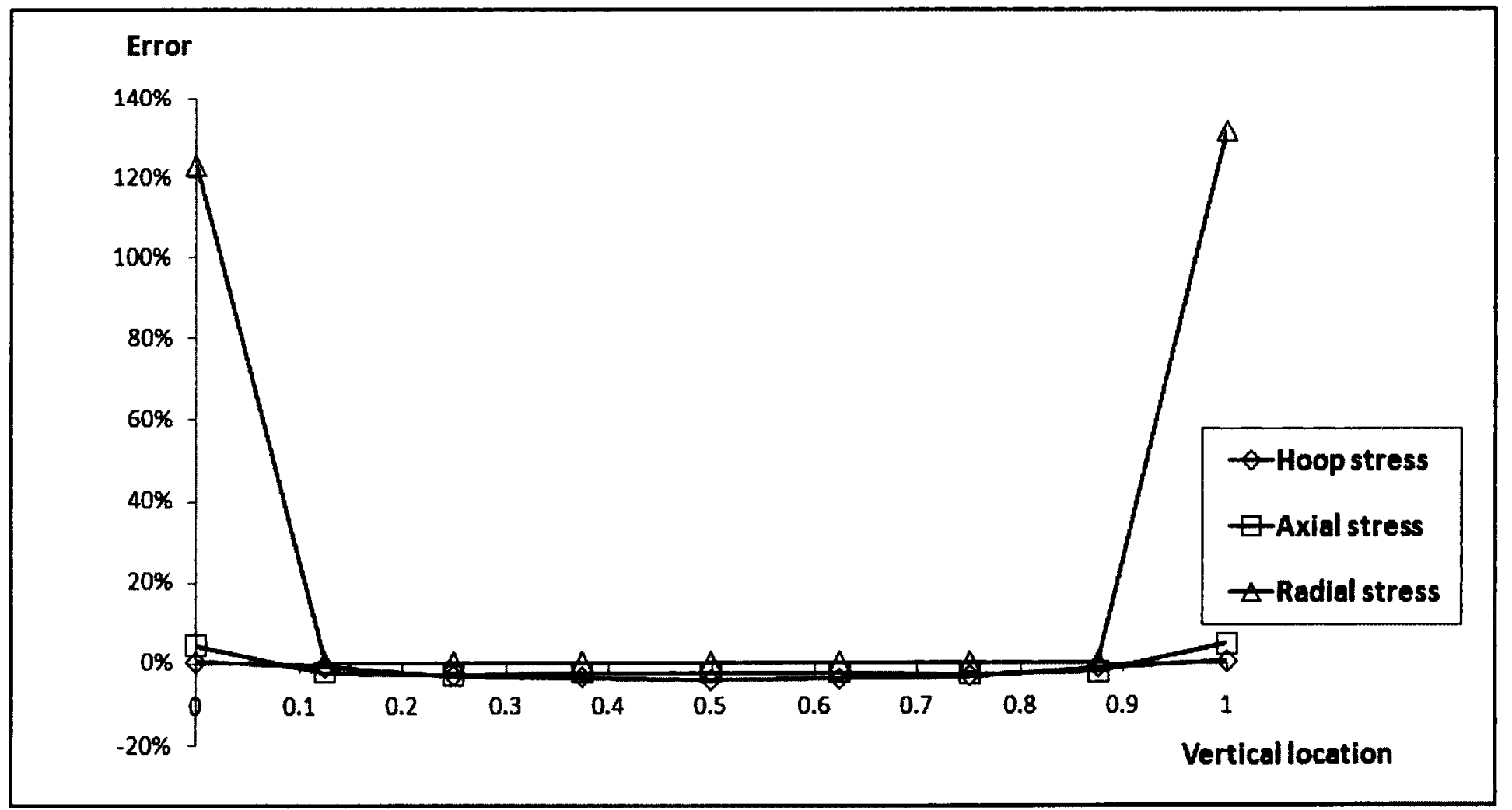

Figure 5.7: Percentage errors in the stresses of the pressurized thin-walled cylinder problem $(t=0.01)$ 


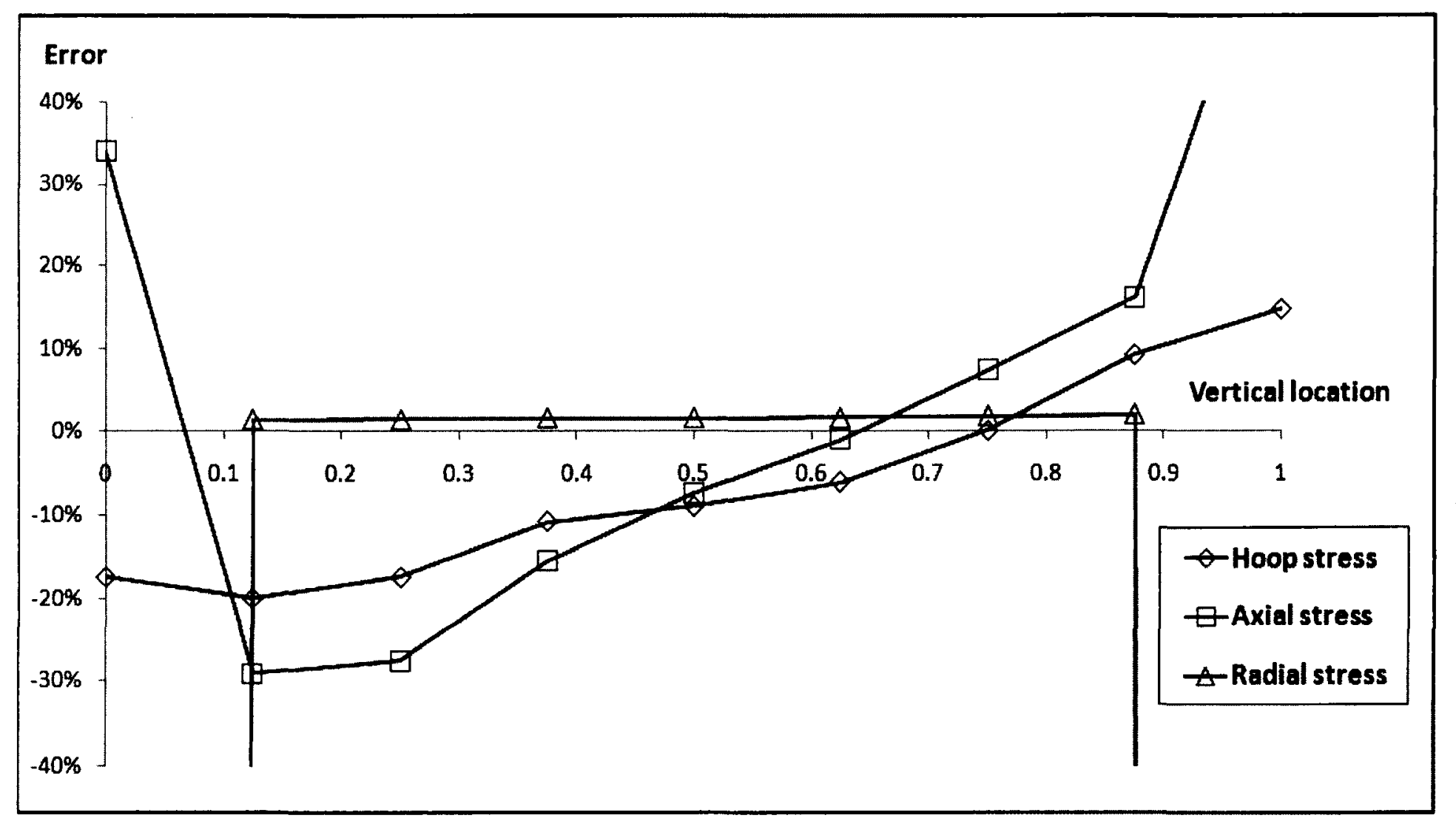

Figure 5.8: Percentage errors in the stresses of the pressurized thin-walled cylinder problem $(t=0.001)$ 


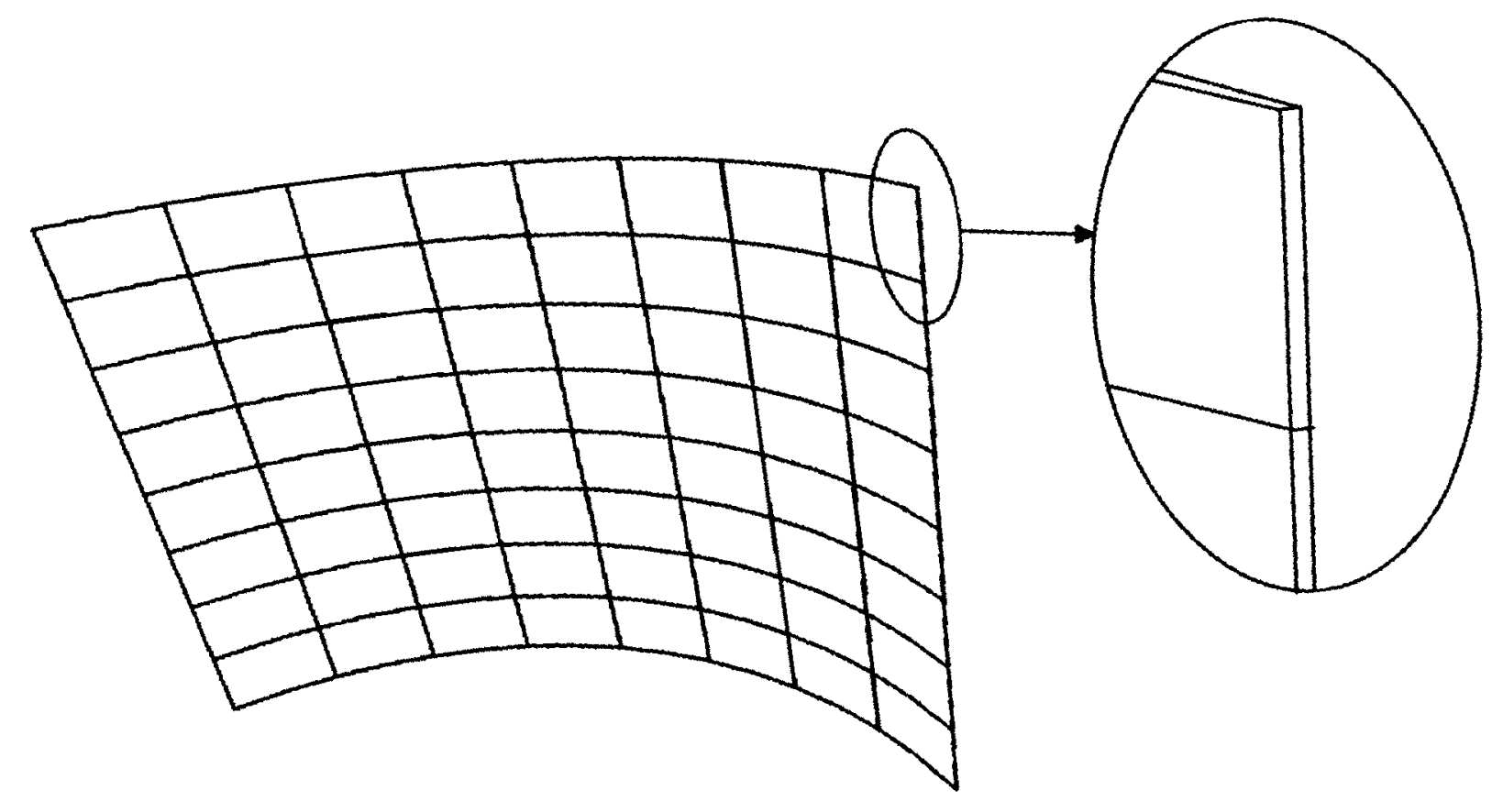

5.9: Refined mesh of the thin-walled cylinder 


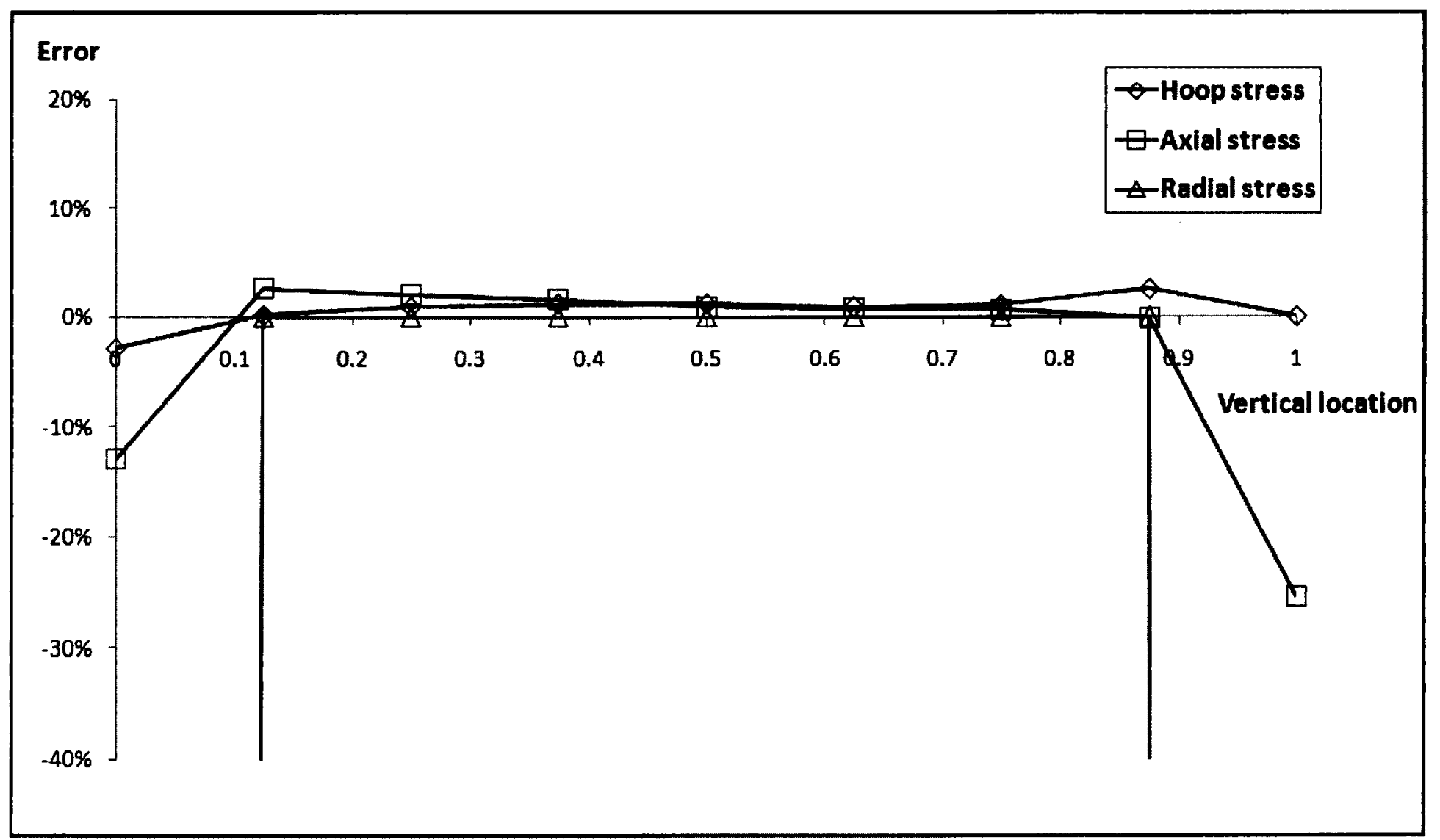

Figure 5.10: Percentage errors in the stresses in the refined BEM mesh for the thin-walled cylinder $(t=0.001)$ 


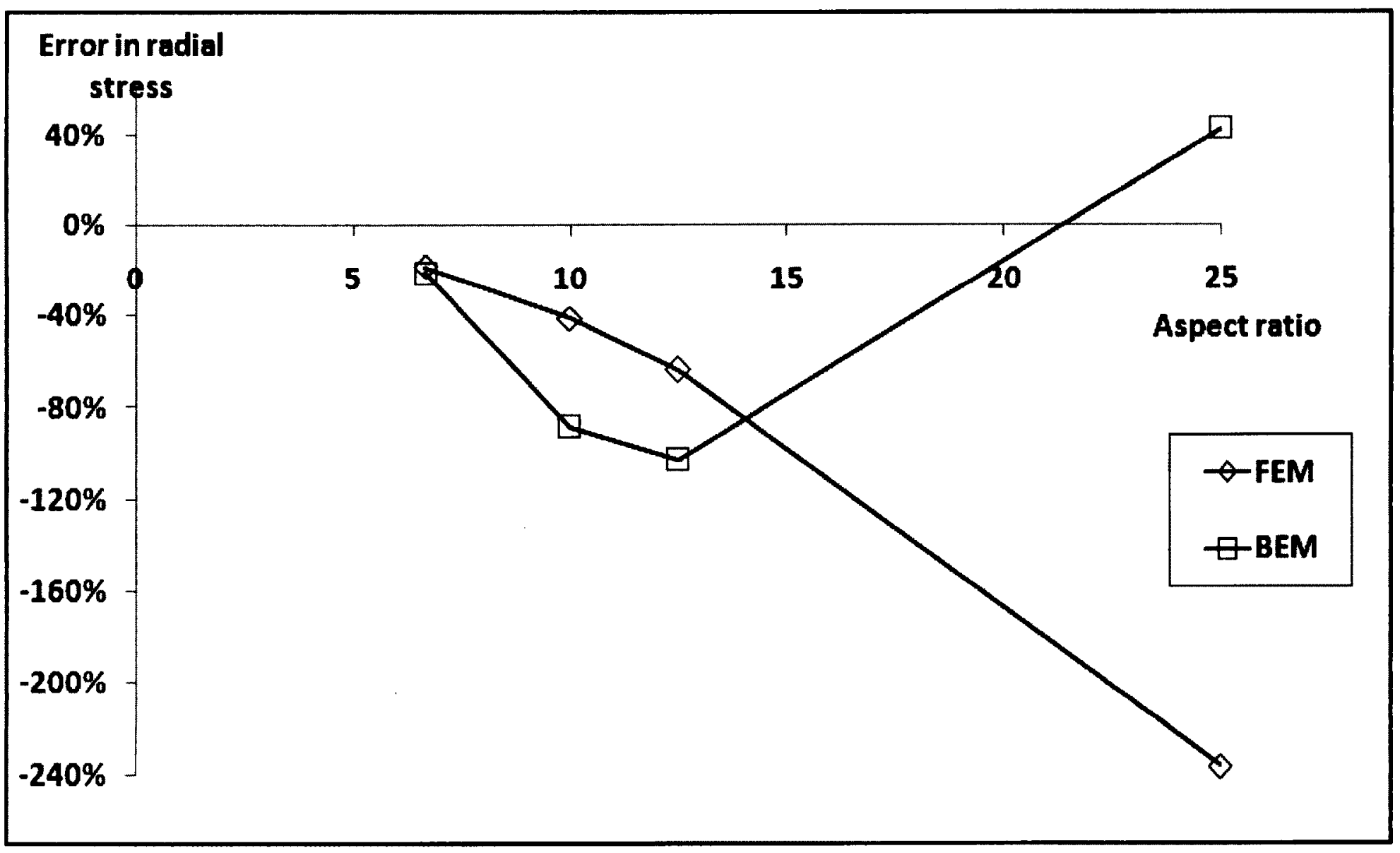

Figure 5.11: Variation of percentage errors in radial stress from BEM and FEM solutions with aspect ratio of elements across thickness 


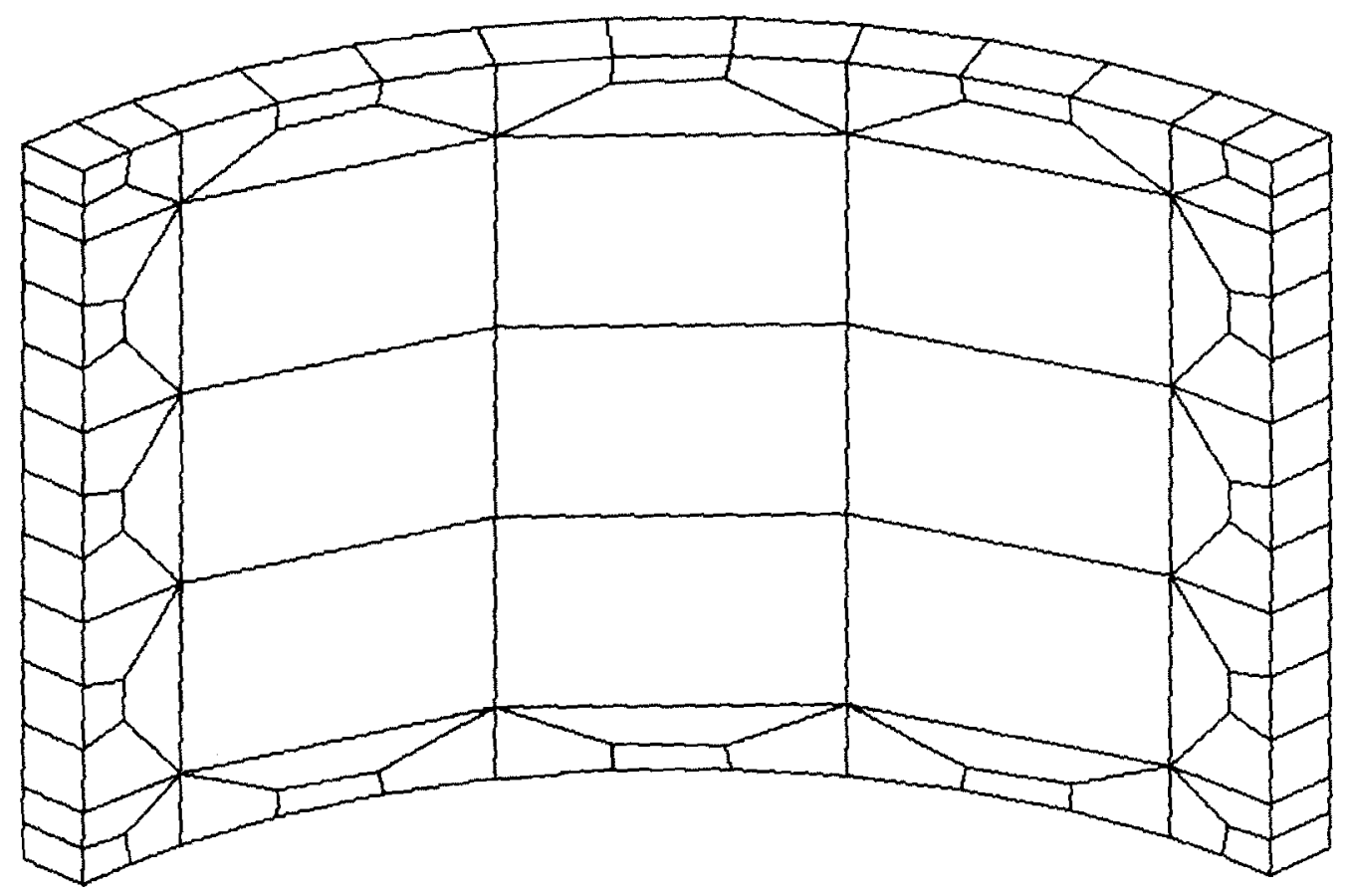

The 5.12. Partially refined mesh for the case of $t=0.1$ 


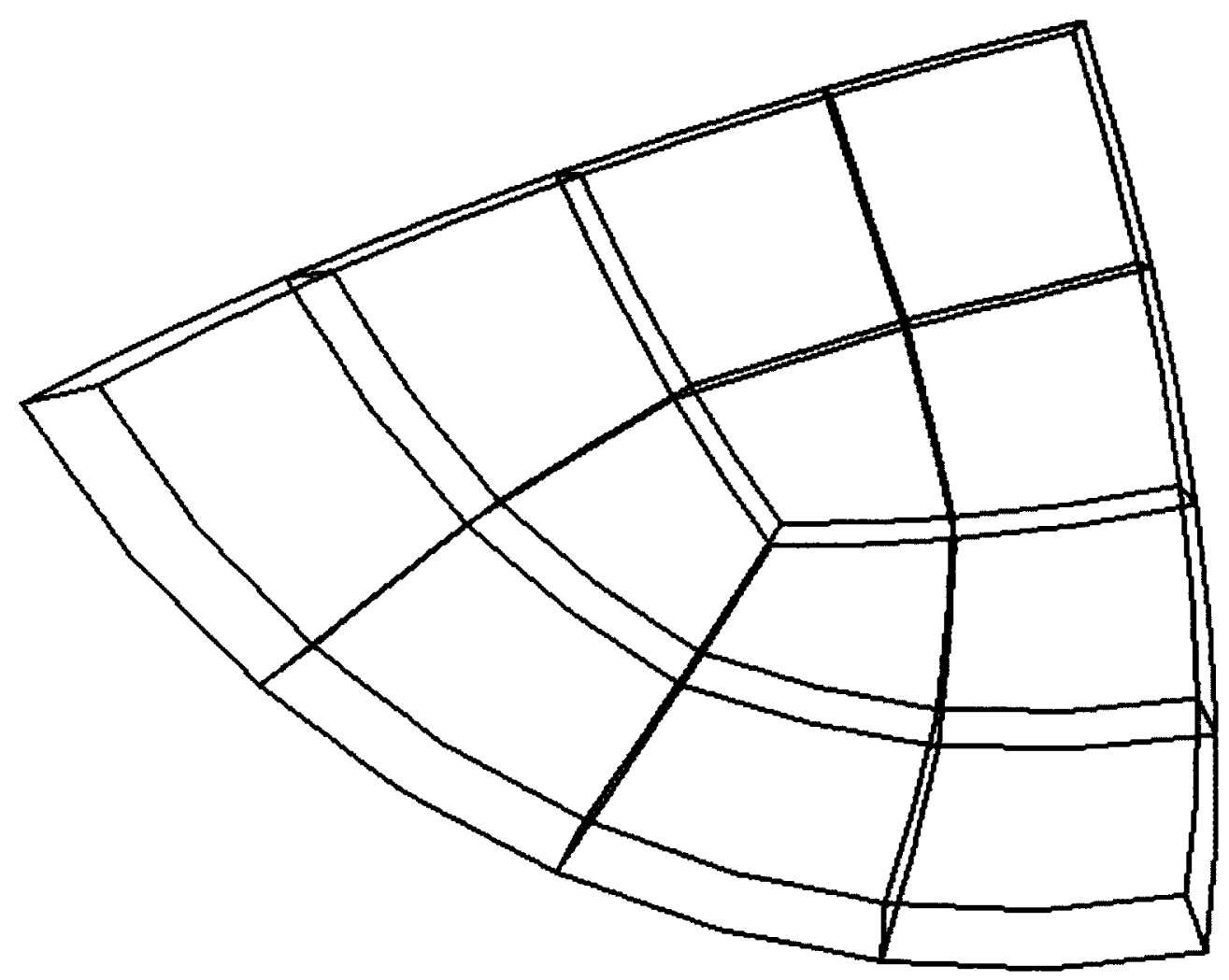

Figure 5.13: BEM mesh of the thin-walled sphere problem 


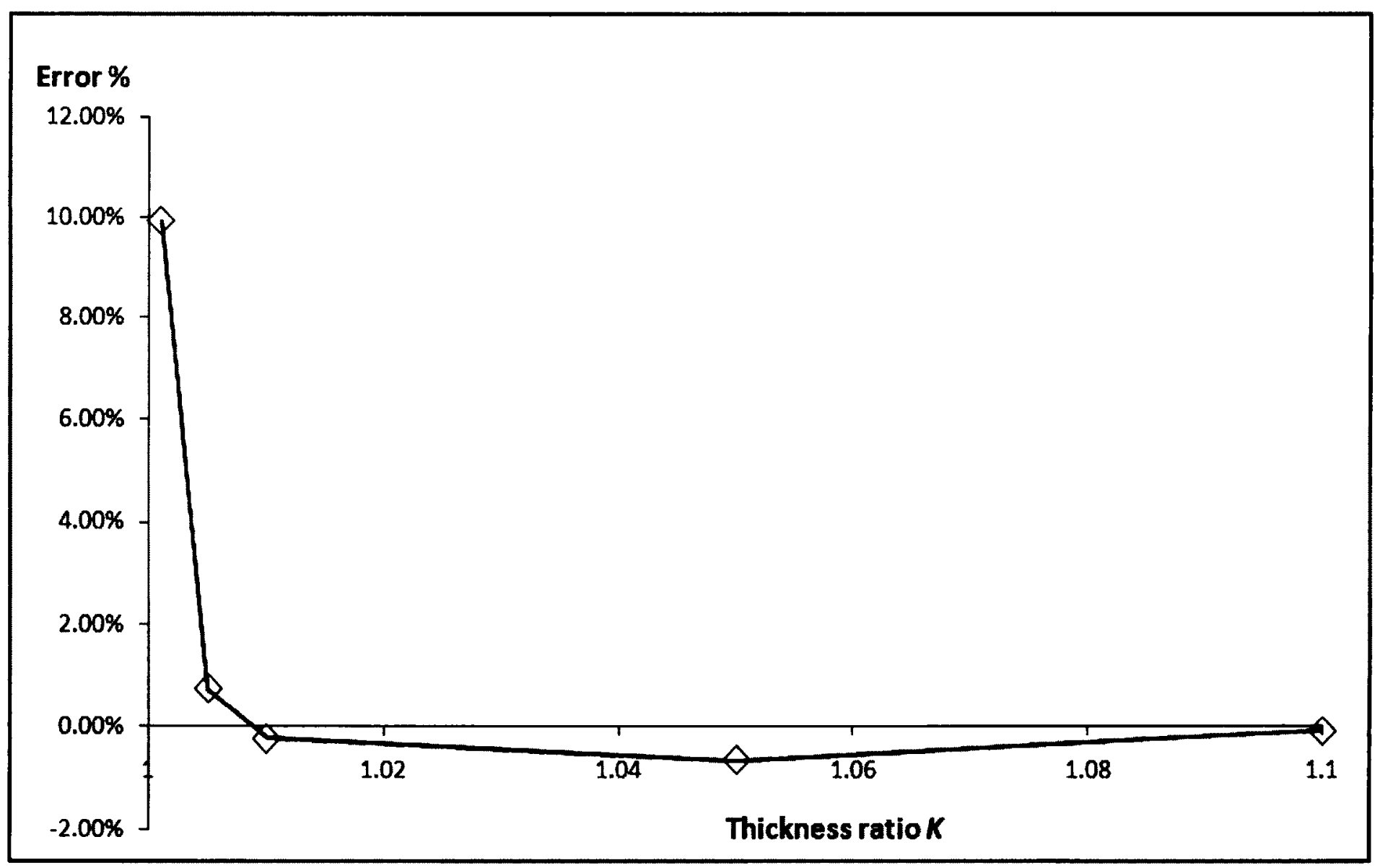

Figure 5.14: Variation of the maximum errors in the circumference stress for the pressurized thin-walled sphere problem with the thickness ratio $K$ 


\section{Chapter 6}

\section{Conclusion}

In this thesis, the self-regularization technique in BEM analysis as proposed in Cruse and Richardson (1996) and Richardson and Cruse (1999) is extended from 2D to 3D elastostatics. Two types of problems involving near singularities of the kernel functions in the integral equation have been treated in this study. They are (i) the evaluation of displacements and stresses at interior points very close to the surface of the domain, and (ii) thin-body problems where two or more parts of the surface are relatively close to one another. The issue arises because the kernel functions contain the fundamental solutions to the governing differential equations in elasticity; they are singular with respect to the distance between the field point and the source point of the domain.

A brief review of the conventional BEM has been provided in Chapter 2. Following this, the self-regularization BEM scheme for numerically evaluating the displacements and stresses at interior points very close to the surface of a $3 \mathrm{D}$ elastic solid has been successfully implemented in this study. The process of the regularization has been described in detail in Chapter 3 and the veracity of the technique and implementation has been demonstrated with several numerical examples. Accurate numerical solutions for such points very close to the surface could not be obtained in 
conventional BEM unless very refined meshes are employed. The usefulness of this scheme may be found in, for example, fracture mechanics analysis for the T-stress at a crack front which requires the computation of contour and domain integrals, or when detailed stress distributions within a domain near a stress concentration is required.

For treating thin-body problems, the self-regularized traction-BIE developed by Richardson and Cruse (1999) for 2D BEM analysis and using a "relaxed continuity" approach, has also been extended to $3 \mathrm{D}$ in this study. It has been found, however, that the discontinuity of the displacement gradients across the inter-element boundaries of the boundary elements remain a serious source of error when using the quadratic conforming elements in the $3 \mathrm{D}$ analysis. An alternative approach involving collocation at points within the element where the $C^{1, \alpha}$ continuity requirement is theoretically met, have also been implemented but yielded only very limited success. For the general case of a problem with corners and non-uniform stress distributions, the traction-BIE implemented has been found not to produce accurate results because of the displacement-gradient discontinuity at the corners; a numerical formulation employing elements which possess this inter-element continuity requirement will need to be employed. Thus the present wholly traction-BIE scheme is not considered to be an efficient one for general 3D analysis.

A "new" self-regularized displacement-BIE has been developed and proposed instead, in Chapter 5, to treat thin-body problems. Using the ideas from the preceding chapters, the scheme involves regularizing the integrals over both parts of the surface of the domain which are very close to each other. With this formulation, the components of the free terms, $C_{i j}$, in the BIE cannot be indirectly obtained numerically by considering the rigid body motion condition as is normally adopted 
in BEM algorithms. Instead, an analytical expression for obtaining the values is implemented; it has been derived in the literature fairly recently. Preliminary tests with some examples show that very accurate results can be obtained with the self-regularized displacement-BIE BEM algorithm with the $C^{0}$ continuous quadratic isoparametric elements that neither the conventional displacement-BIE nor the self-regularized traction-BIE have been able to achieve with. This is especially true for the results at smooth surfaces. For problems with external corners, care should be exercised in the numerical modeling to ensure that the aspect ratio of the elements around the corners are not too large so that accurate results could still be obtained.

\subsection{Suggestions for Future Work}

(a) Clearly, a more extensive range of tests involving more complex boundary conditions and geometries need to be carried out to firmly establish the veracity of the displacement-BIE scheme developed in Chapter 5. This could include discontinuous load conditions over parts of the solid surface as well as different constraint conditions. The geometries can also be extended to those varying from thick cross-sections to thin ones. Some criterion could be developed to establish when the self-regularization scheme is required in the BEM algorithm.

(b) Further investigation is also needed to reduce the effect of large aspect ratio of the boundary element around corners on the solution accuracy instead of requiring significant mesh refinement there.

(c) The self-regularization BEM scheme can also be extended to treat thermoelastic problems and those involving body forces. 


\section{References}

A.A. Becker. The boundary element method in engineering : a complete course. McGraw-Hill, 1992.

C. C. Chien, H. Rajiyah, and S. N. Atluri. On the evaluation of hyper-singular integrals arising in the boundary element method for linear elasticity. Computational Mechanics, 8(1):57-70, 1991.

T. A. Cruse and R. Aithal. Non-singular boundary integral equation implementation. International Journal for Numerical Methods in Engineering, 36:237-254, 1993.

T. A. Cruse and J. D. Richardson. Non-singular Somigliana stress identities in elasticity. International Journal for Numerical Methods in Engineering, 39(19):3273-3304, 1996.

T. A. Cruse and W. Suwito. On the Somigliana stress identity in elasticity. Computational Mechanics, 11(1):1-10, 1993.

P. Dangla, J. F. Semblat, H. Xiao, and N. Delepine. A simple and efficient regularization method for 3D BEM: Application to frequency-domain elastodynamics. Bulletin of the Seismological Society of America, 95(5):1916C1927, 2005.

J. Dominguez and M.P. Ariza. A direct traction-BIE approach for three-dimensional crack problems. Engineering Analysis with Boundary Elements, 24:727-738, 2000. 
J. Dominguez, M. P. Ariza, and R. Galleg. Flux and traction boundary elements without hypersingular or strongly singular integrals. International Journal for $\mathrm{Nu}$ merical Methods in Engineering, 48:111-135, 2000.

R. Gallego and J. Dominguez. Hypersingular BEM for transient elastodynamics. International Journal for Numerical Methods in Engineering, 39:1681-1705, 1996.

Z.R. Jin, D.M.Zhang, Y.H.Wang, and Z.L.Li. Dynamic element discretization method for solving 2D traction boundary integral equations. Engineering Analysis with Boundary Elements, 35(11):1204-1213, 2011.

A. B. Jorge, T. A. Cruse, T. S. Fisher, and G. O. Ribeiro. A new variational self-regular traction-BEM formulation for inter-element continuity of displacement derivatives. Computational Mechanics, 32(4-6):401-414, 2003.

L. Jun, G. Beer, and J.L. Meek. Efficient evaluation of integrals of order $1 / r, 1 / r^{2}, 1 / r^{3}$ using gauss quadrature. Engineering Analysis, 2(3):118-123, 1985.

J. H. Kane. Boundary element analysis in engineering continuum mechanics. Englewood Cliffs, N.J. : Prentice Hall, 1994.

G. Krishnasamy, F. J. Rizzo, and Y. J. Liu. Boundary integral equations for thin bodies. International Journal for Numerical Methods in Engineering, 37:107-121, 1994.

J. C. Lachat and J. O. Watson. Effective numerical treatment of boundary integral equations. International Journal for Numerical Methods in Engineering, 10:991$1005,1976$.

J. Li. The evaluation of T-stress using boundary element method. M.A.Sc. thesis, Carleton University, 2003. 
Sh. D. Li and Q. B. Huang. An improved form of the hypersingular boundary integral equation for exterior acoustic problems. Engineering Analysis with Boundary Elements, 34:189-195, 2010.

Y. J. Liu. Elastic stability analysis of thin plate by the boundary element method-a new formulation. Engineering Analysis, 4:160-164, 1987.

Y. J. Liu and Sh. H. Chen. A new form of the hypersingular boundary integral equation for 3-D acoustics and its implementation with $C^{0}$ boundary elements. Computer Methods in Applied Mechanics and Engineering, 173:375-386, 1999.

Y. J. Liu and F.J. Rizzo. A weakly singular form of the hypersingular boundary integral equation applied to 3-D acoustic wave problems. Computer Methods in Applied Mechanics and Engineering, 96:271-287, 1992.

Y.J. Liu. Analysis of shell-like structures by the boundary element method based on 3-D elasticity: formulation and verification. International Journal for Numerical Methods in Engineering, 41(6):541-558, 1998.

H. Ma and N. Kamiya. A general algorithm for accurate computation of field variables and its derivatives near the boundary in BEM. Engineering Analysis with Boundary Elements, 25(10):833-841, 2001.

P. A. Martin and F. J. Rizzo. Hypersingular integrals: how smooth must the density be? International Journal for Numerical Methods in Engineering, 39:687-704, 1996.

P. A. Martin, F. J. Rizzo, and T. A. Cruse. Smoothness-relaxation strategies for singular and hypersingular integral equations. International Journal for Numerical Methods in Engineering, 42:885-906, 1998. 
Y. Mi and M.H. Aliabadi. Dual boundary element method for three-dimensional fracture mechanics analysis. Engineering Analysis with Boundary Elements, 10(2): $161-17,1992$.

Z. Niu, W.L. Wendland, X. Wang, and H. Zhou. A semi-analytical algorithm for the evaluation of the nearly singular integrals in three-dimensional boundary element methods. Computer Methods in Applied Mechanics and Engineering, 194(9-11): 1057-1074, 2005.

Walter D. Pilkey. Peterson's stress concentration factors [electronic resource]. Hoboken, N.J. : John Wiley, 2008.

G. O. Ribeiro, T. S. A. Ribeiro, A. B. Jorge, and T. A. Cruse. Evaluation of nonsingular BEM algorithms for potential problems. Journal of the Brazilian Society of Mechanical Sciences and Engineering, 31(3):261-268, 2009.

T. S. A. Ribeiro, G. O. Ribeiro, and A. B. Jorge. On the relaxed continuity approach for the selfregular traction-BIE. Mecanica Computacional, Vol. XXI:1263-1281, 2002.

J. D. Richardson. Weakly singular BEM for fracture modeling. PhD thesis, Vanderbilt University, 1998.

J. D. Richardson and T. A. Cruse. Weakly singular stress-BEM for 2D elastostatics. International Journal for Numerical Methods in Engineering, 45:13-35, 1999.

T. J. Rudolphi. The use of simple solutions in the regularization of hypersingular boundary integral equations. Mathematical and Computer Modelling, 15:269-278, 1991.

P. D. Shah. Determination of T-stress solutions for cracked $2 D$ anisotropic elastic bodies using the boundary element method. PhD thesis, Carleton University, 2007. 
P. D. Shah, C. L. Tan, and X. Wang. T-stress solutions for two-dimensional crack problems in anisotropic elasticity using the boundary element method. Fatigue $\mathcal{E}$ Fracture of Engineering Materials \& Structures, 29(5):343-356, 2006.

V. Sladek, J. Sladek, and M. Tanaka. Regularization of hypersingular and nearly singular integrals in the potential theory and elasticity. International Journal for Numerical Methods in Engineering, 36(10):1609-1628, 1993.

C. L. Tan. The boundary element method: a short course. Department of Mechanical \& Aerospace Engineering, Carleton University, Ottawa, Canada, 1987.

C.L. Tan and R.T. Fenner. Three-dimensional stress analysis by the boundary integral equation method. Journal of Strain Analysis, 13:213-219, 1978.

Ch. Wang and Z. L. Li. Application of relations of singularity intensities of tangent derivatives of boundary displacements and tractions to BEM. Engineering Analysis with Boundary Elements, 33:618-626, 2009.

G. Zh. Xie, J.M. Zhang, X. Y. Qin, and G. Y. Li. New variable transformations for evaluating nearly singular integrals in 2D boundary element method. Engineering Analysis with Boundary Elements, 35(6):811-817, 2011.

T. Q. Ye and Y. J. Liu. Finite deflection analysis of elastic plate by the boundary element method. Applied Mathematical Modelling, 9:183-188, 1985.

J. Yu. T-stress solotions of cracks emenating from circular holes. M.A.Sc. thesis, Carleton University, Ottawa, Ontario, Canada, 2006.

H.L. Zhou, Z.R. Niu, C. Z. Cheng, and Z.W. Guan. Analytical integral algorithm applied to boundary layer effect and thin body effect in BEM for anisotropic potential problems. Computers and Structures, 86:1656-1671, 2008. 